\title{
Microstructure modeling and finite element analysis of particulate reinforced metal matrix composites
}

Praveen Shankaranarayana

West Virginia University

Follow this and additional works at: https://researchrepository.wvu.edu/etd

\section{Recommended Citation}

Shankaranarayana, Praveen, "Microstructure modeling and finite element analysis of particulate reinforced metal matrix composites" (2006). Graduate Theses, Dissertations, and Problem Reports. 4268. https://researchrepository.wvu.edu/etd/4268

This Thesis is protected by copyright and/or related rights. It has been brought to you by the The Research Repository @ WVU with permission from the rights-holder(s). You are free to use this Thesis in any way that is permitted by the copyright and related rights legislation that applies to your use. For other uses you must obtain permission from the rights-holder(s) directly, unless additional rights are indicated by a Creative Commons license in the record and/ or on the work itself. This Thesis has been accepted for inclusion in WVU Graduate Theses, Dissertations, and Problem Reports collection by an authorized administrator of The Research Repository @ WVU. For more information, please contact researchrepository@mail.wvu.edu. 
Microstructure Modeling and Finite Element Analysis of Particulate Reinforced Metal Matrix Composites

\title{
By
}

Praveen Shankaranarayana

\author{
A THESIS \\ Submitted to the College of Engineering and Mineral Resources \\ at \\ West Virginia University \\ in partial fulfillment of the requirements \\ for the degree of \\ Master of Science \\ in \\ Mechanical Engineering
}

\author{
Samir N. Shoukry, Ph.D, Co-Chair \\ Jacky Prucz, Ph.D, Co-Chair \\ Kenneth H. Means, Ph.D \\ Darran Cairns, Ph.D \\ Gergis William, Ph.D
}

Department of Mechanical and Aerospace Engineering

Morgantown, West Virginia

2006 


\section{Abstract \\ Microstructure Modeling and Finite Element Analysis of Particulate Reinforced Metal Matrix Composites}

\section{Praveen Shankaranarayana}

The study of microscopic and macroscopic response of a particulate reinforced metal matrix composites (PRMMC) using finite element (FE) analysis is the aim of the current investigation. In this regard, three types of microstructure models are subjected to FE analysis. In the first part of the work, a technique is presented for the generation of artificial microstructures containing spherical and ellipsoid shaped inclusions. The problem of detection of ellipsoidal intersection is tackled using newly available algorithms. To account for higherscale effects such as clustering, information from the micro-scale model is input into a meso-scale model.

The FE analysis of the artificial microstructure and a summary of the results form the second part of the study. It is seen that the newly developed models agree very well with the published results and that the microstructure generation technique can be reused in many computational micromechanics problems with minimum modifications. Finally, the results obtained from the new models are used to study a problem of practical significance, namely bolted joints made of MMC material. 


\section{Acknowledgements}

This thesis would not have been possible without the help of many people. First, I would like to thank my advisor, Dr. Samir Shoukry, for his support, guidance and advice throughout the endeavor. His belief in me has been a constant source of motivation. I would like to express my sincere gratitude to Dr. Jacky Prucz, for his constant encouragement throughout this research.

My gratitude goes towards Dr. Gergis William for his patience and help with FEA. He has been a constant source of new ideas, even when the situation seemed hopeless.

I would like to thank my research team - Dhananjay, for all the technical discussions we had over the many months and Thomas, for his help with ellipsoidal modeling.

My thanks to all my friends - Mahesh, Shashi, Ashwin, Karthik, Amar, Deepika and Bala for their encouragement and support throughout.

Finally, my gratitude to my family, whose belief in me has been a valuable asset not only in this endeavor, but also in every aspect of my life. 


\section{Table of Contents}

Abstract................................................................

Acknowledgements................................................ iii

Table of Contents ........................................................

List of Figures ...................................................

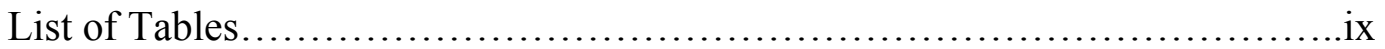

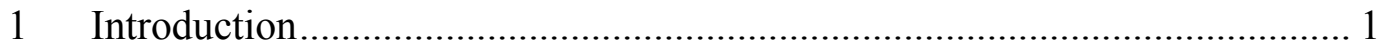

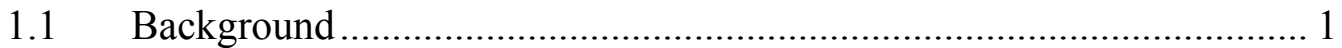

1.2 Analytical modeling of composites .............................................. 1

1.2.1 Models derived using variational principle.................................... 2

1.2.2 Models assuming ideal microstructure geometry ........................... 3

1.2.3 Approximate Models ..................................................................... 4

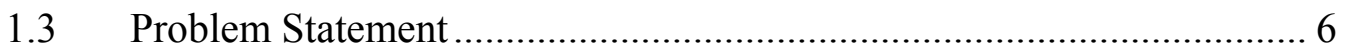

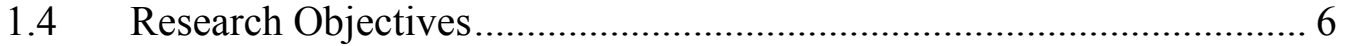

1.5 Organization of the thesis ........................................................... 7

2 Literature review of microstructure modeling and testing of metal matrix

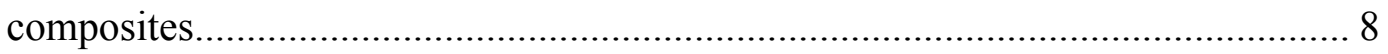

2.1 Microstructure Modeling ............................................................ 8

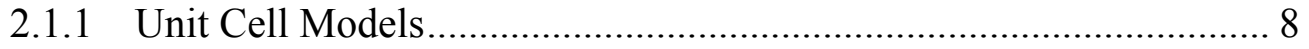

2.1.2 Voronoi Cell Models....................................................................... 14

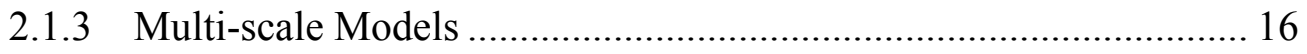

2.1.4 Summary of Micromechanical Models.......................................... 21

2.2 FE modeling and testing of bolted joints ...................................... 21

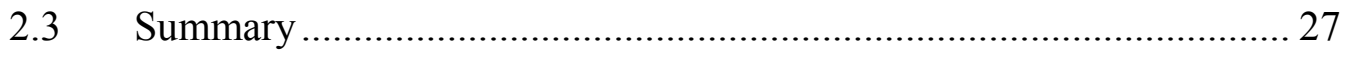

3 Finite Element Modeling of Microstructures............................................ 28

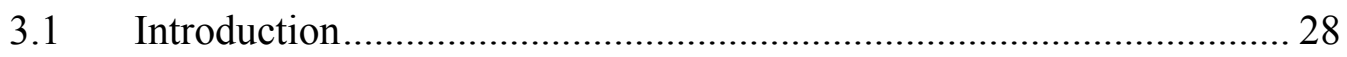

3.2 Body centered cubic UCM.......................................................... 29

3.3 RVE with randomly distributed spheres ....................................... 31 
3.4 RVE with randomly distributed ellipsoids............................................... 36

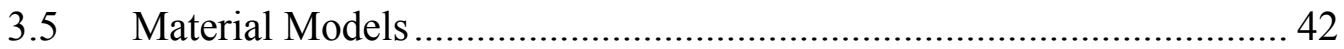

3.6 Boundary conditions and loading ........................................................ 43

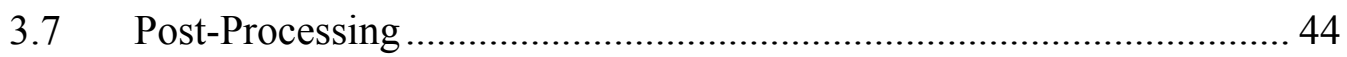

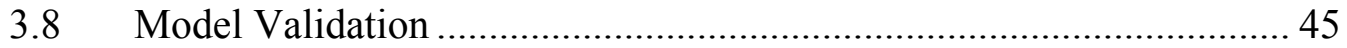

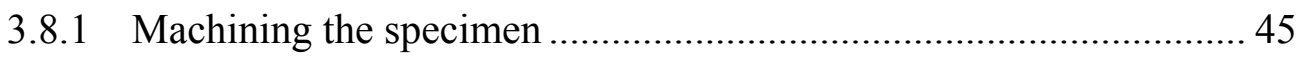

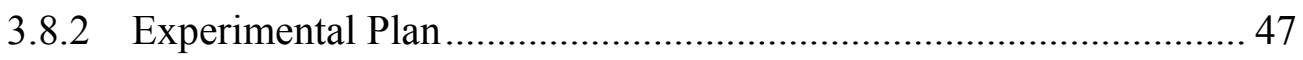

3.8.3 Test Procedure ....................................................................... 48

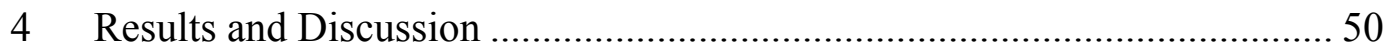

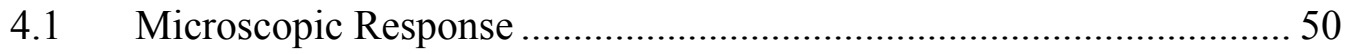

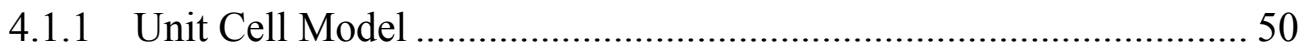

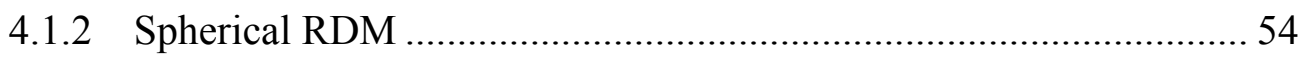

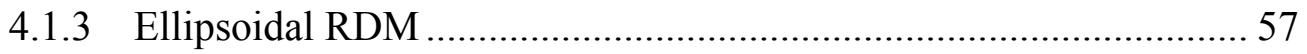

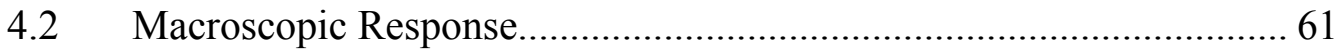

5 Mesoscale modeling and experimental evaluation of MMC material .......... 67

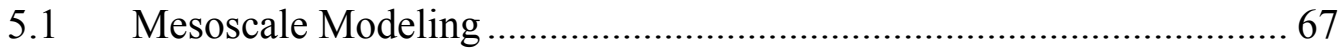

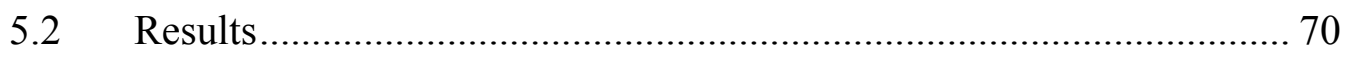

5.2.1 Effect of Particle Clustering........................................................ 72

6 Experimental testing and FE modeling of MMC bolted joints...................... 74

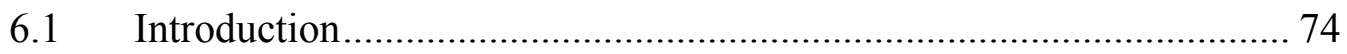

6.2 Experimental evaluation ................................................................... 74

6.3 Finite element modeling …………………………............................. 77

$6.4 \quad$ Results and Discussion ...................................................................... 78

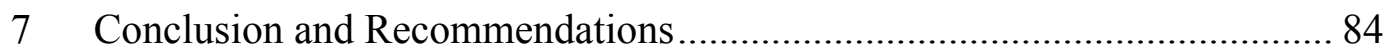

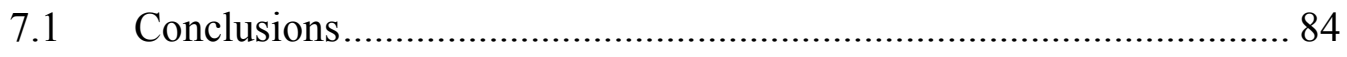

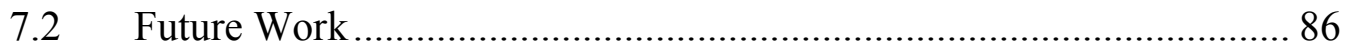




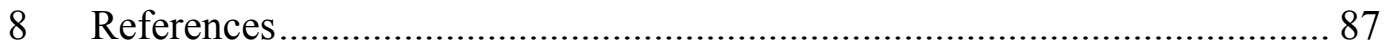

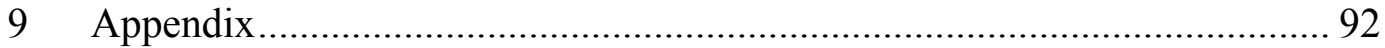

9.1 Matlab routine to generate microstructures with sphere-shaped

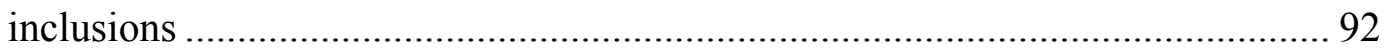

9.2 Matlab routine to generate microstructures with ellipsoid-shaped

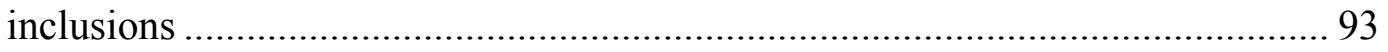

9.3 Example ANSYS macro file used to specify material properties, loading, boundary conditions and post processing .............................................. 96 


\section{List of Figures}

Figure 2.1 (a) Optical micrograph of a section having 10\% inclusion fraction (b) Voronoi cell tessellation of the equivalent microstructure. ${ }^{[15]}$.............................................. 15

(a) along the extrusion direction $\quad$ (b) perpendicular to the protrusion direction .......... 17

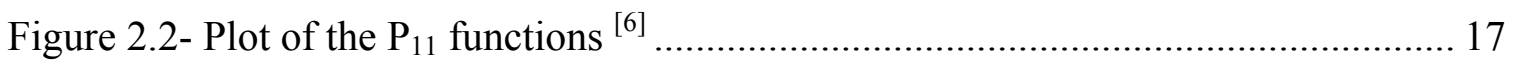

Figure 2.3 - Equivalent microstructures for inclusions ${ }^{[7]}$........................................ 19

Figure 2.4 - The part of the bolted flange modeled using finite elements. ${ }^{[25]}$................. 23

Figure 25 - Experimental setup with extensometer for testing single-shear bolted joints [27] 25

Figure 3.2 A FE mesh for a UCM with 15\% volume fraction...................................... 31

Figure 3.3 An SEM image of the microstructure of the Al/SiC MMC being studied... 32

Figure 3.4 The algorithm for generation of RDM having spherical inclusions............. 34

Figure $3.5 \mathrm{RDM}$ of $20 \%$ volume fraction having spheres of average radius $9 \mu \mathrm{m}$ as

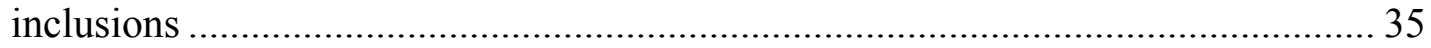

Figure 3.6 Meshed spehrical inclusions of a 20\% volume fraction microstructure.......... 36

Figure 3.7 A prolate ellipsoid which has the two minor radii equal to each other .......... 37

Figure 3.8 The above flowchart summarizes the subroutine used to generate microstructure containing ellipsoid shaped inclusions.

Figure 3.9 An example microstructure containing ellipsoidal inclusions and having volume fraction of $15 \%$.

Figure 3.10 Meshed ellipsoidal inclusions of a 15\% volume fraction microstructure. Note that the matrix surrounding the inclusions is not shown in the figure.

Figure 3.11 Schematic of loading and boundary conditions imposed on the models...... 43

Figure 3.12 - The EDM machine used to reduce the MMC bricks to the required

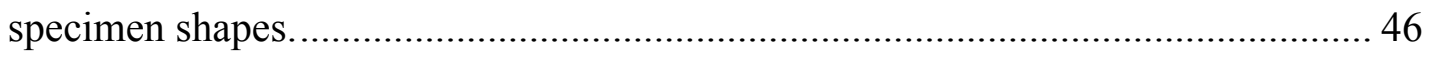

Figure 3.14 - Dimensions of the specimen used for three point bending tests. All

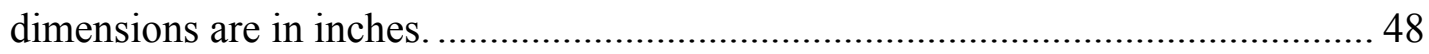

Figure 3.15 - Three point bending testing of MMC specimen using an INSTRON machine. 48

Figure 4.1- Distribution of von-Mises stress (MPa) in a 20\% volume fraction UCM .... 51

Figure 4.3 - Distribution of von-Mises equivalent stress in 15\% volume fraction UCM. 52 
Figure 4. 4 - Distribution of von-Mises equivalent strains in 20\% volume fraction UCM.

Figure 4.5- Distribution of von-Mises equivalent strains in 15\% volume fraction UCM.53

Figure 4.6 - A section of $15 \%$ volume fraction RDM with spherical inclusions, showing the distribution of von-Mises equivalent stress. 54

Figure 4.7 - A section of 15\% volume fraction RDM with spherical inclusions, showing the distribution of maximum principal stress. 55

Figure 4.8 - Distribution of von-Mises equivalent strain in the $15 \%$ volume fraction RDM. 56

Figure 4.9 - Distribution of the first principal stress in the $20 \%$ volume fraction RDM.. 56 Figure 4.10 - Distribution of von-Mises equivalent stress in a 20\% volume fraction RDM

Figure 4.11 - Variation of the von Mises and principal stresses in the matrix around the reinforcement. 58

Figure 4.12 - A section through the ellipsoidal RDM model (20\% volume fraction) showing the distribution of von-Mises effective stress.

Figure 4.13 - A section through the ellipsoidal RDM model (15\% volume fraction) showing the distribution of von-Mises effective stress.

Figure 4.14 - Von-Mises effective stress in a typical spherical and ellipsoidal particle, showing the higher stress concentration induced in the ellipsoidal particle. 60

Figure 4.15 - The variation of Young's modulus of the composite with the volume fraction.

Figure 4.16 - The predicted and reported stress-strain curves for a $20 \%$ volume fraction $\mathrm{MMC}$

Figure 4.17 - Stress-strain curves for a 15\% volume fraction MMC as predicted by various FE models, compared with the experimental values.

Figure 4.18 - Comparison of values obtained for $20 \% \mathrm{SiC}$ material from ellipsoidal RDM model and experiments.

Figure 5.1 - Probability density function for normal distribution having $\mu=0$. 68

Figure 5.2 - The number of elements having various volume fractions in a 8000 element mesoscale model 
Figure 5.3 - Mesoscale random array with 20\% volume fraction and 0.1 SD.

Figure 5.4 - Stress-strain curve from the random array mesoscale model and the reported curve in the literature for $20 \%$ volume fraction material..................................... 71

Figure 5- 5 - Stress-strain curve from experiments and numerical models for $15 \%$ volume fraction material.

Figure 5.6 - Stress-strain curves from mesoscale models having different standard deviations of local volume fractions. .

Figure 6.1 - A standard bolted joint shown with the parameters which are used to describe the joint. 75

Figure 6. 2 - Dimensions of specimen used in the bolted joint testing. 75

Figure 6.3 - Schematic of the test fixture used in the bolted joint testing of MMC specimen 76

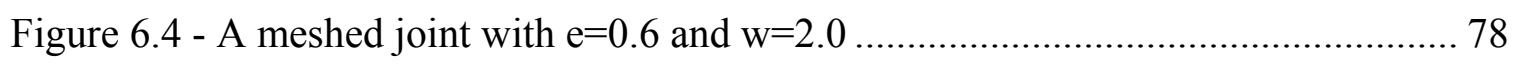

Figure 6.5 - Load -displacement curves for various joints....................................... 79

Figure 6.6 - Variation of gripping force with axial load......................................... 80

Figure 6.7 - Failure Modes of MMC Joints. .......................................................... 81

Figure 6.8 - Distribution of $\mathrm{xy}$ in the joint prior, at and post failure.................82 Figure 6.9 - Distribution of von-Mises effective stress for a specimen having e $=2.5$ and $\mathrm{w}$ $=1.5$ and $20 \% \mathrm{SiC}$ as reinforcement before and after failure initiation..................83

\section{List of Tables}

Table 3.1 Radii of inclusions for different volume fractions of BCC-UCM ................. 29

Table 3-2 Properties of matrix and inclusion materials used in the FE model ................ 43

Table 4.1 - Material properties as predicted by the three microstructure models............. 62

Table $4.2-0.2 \%$ yield strength as predicted by the two models and their experimentally determined values. 626 


\section{Chapter One}

\section{Introduction}

\subsection{Background}

Metal matrix composites (MMC) despite their superior mechanical properties, have seen limited use in mainstream industries. This is due to the high fabrication and processing costs which are traditionally associated with MMCs. But with the introduction of liquid-state processing techniques for production, low-cost discontinuously reinforced aluminum (DRA) has become a reality in recent years. This has lead to several successful applications of these materials in the automobile and electronics industries.

DRA exhibits several advantages over conventional aluminum alloys such as high strength-to-weight ratio, excellent thermal properties and high wear resistance. Additionally, they can be machined using conventional techniques. By varying the processing parameters during the time of production, the properties of the microstructure of the DRA, such as reinforcing particle size, matrix strength, residual stresses in the matrix, can be varied. If the influence of these microstructural features on the overall stress-strain response of the composite is known, it is possible, within limits, to tailor the DRA to the application at hand. Unfortunately, the way in which the microstructural features affect the macroscopic response is not fully understood.

\subsection{Analytical modeling of composites}

Since testing of various kinds of MMCs with varied microstructures is both time consuming and expensive, computational studies are required to effectively characterize the microstructures and predict their response - both plastic and elastic. Analytical models have been used extensively to estimate the elastic properties of the particulate reinforced metal matrix composites (PRMMCs). Almost all models assume that the composite is a heterogeneous material containing two phases, each phase being represented as an isotropic continuum. The analytical models in use today can be classified based on their main characteristics, as follows ${ }^{[9]}$ 


\subsubsection{Models derived using a variational principle}

This group contains the Voigt-Reuss (V-R) bounds and the Hashin-Shtrikman bounds which are derived from the variational principal ${ }^{[32]}$. The Voigt-Reuss bounds were obtained by using a combination of the theorem of minimum potential energy and the theorem of least work to determine the lower and upper bound of elastic constanr respectively. The expressions given below are assumed to be valid for any kind of composite regardless of the shape or size of the reinforcing phase.

$$
\begin{aligned}
& E_{c}^{u}=E_{m} V_{m}+E_{r} V_{r} \\
& E_{c}^{l}=\left[\frac{V_{m}}{E_{m}}+\frac{V_{r}}{E_{r}}\right]^{-1}
\end{aligned}
$$

where $E_{m}, E_{r}$ are the stiffness of the matrix and reinforcement respectively $\mathrm{V}_{\mathrm{m}}, \mathrm{V}_{\mathrm{r}}$ are the volume fractions of the matrix and reinforcement and $\mathrm{E}_{\mathrm{c}}{ }^{\mathrm{u}}, \mathrm{E}_{\mathrm{c}}{ }^{1}$ are the upper and lower limits of the composite stiffness as predicted by the model.

Eq. (1) also known as the rule of mixture (ROM), while Eq. (2) is the inverse rule of mixture. It is known that while the V-R bounds are reasonably accurate in predicting the stiffness of composites, they are fall well short in strength predictions.

The Hashin-Shtrikman (H-S) bounds are also derived from variational principles and provide much tighter bounds on the bulk and shear modulus of the composite ${ }^{[32]}$. The Young's modulus is obtained from the bulk and shear modulus using relations applicable to isotropic materials. Thus, for these bounds to be used, the composite in question must be statistically isotropic, such is the case of particulate reinforced composites. The H-S bounds are given by:

$$
K_{c}^{l}=K_{m}+\frac{V_{r}}{\frac{1}{K_{r}-K_{m}}+\frac{3 V_{m}}{3 K_{m}+4 G_{m}}}
$$




$$
G_{c}^{l}=G_{m}+\frac{1}{\frac{1}{G_{r}-G_{m}}+\frac{6 V_{m}\left(K_{m}+2 G_{m}\right)}{5 G_{m}\left(3 K_{m}+4 G_{m}\right)}}
$$

where $\mathrm{G}$ and $\mathrm{K}$ stand for the shear and bulk modulus, respectively, and the subscripts $\mathrm{m}, \mathrm{c}$ and $\mathrm{r}$ denote the matrix, composite and reinforcement, respectively.

The upper H-S bounds are obtained by interchanging $\mathrm{m}$ and $\mathrm{r}$ subscripts in the above two equations. Since the H-S bounds are much closer to the experimental values and are also easy to compute, they are used as a standard for verifying any new model or theory. In this study, a comparison of the new model's predictions with the H-S bounds is presented.

\subsubsection{Models assuming ideal microstructure geometry}

This class of models treats the microstructure of the composite as being made of ideally shaped and equally spaced inclusions - in the form of spheres, cylinders or ellipses. A representative volume element (RVE), which is defined as the smallest part of the microstructure which can considered as statistically representative of the composite, is utilized in deriving expressions for material response. The periodic array model assumes that the inclusions are arranged at regular intervals throughout the composite and defines a unit cell to be analyzed either analytically or using finite element analysis. The arrangement of the inclusions in the matrix can be assumed to be in the shape of either hexagonal or square packing. The overall properties of the composite are different for different arrays having the same volume fraction.

The unit cell used in the periodic array model contains $1-5$ reinforcing particles embedded in the matrix. The radius of the particles is varied to achieve the required volume fraction in the unit cell, which is equal to the overall volume fraction of the composite. One of the important parameters of the cell is the aspect ratio, which is the ratio of the length of the cell to the diameter of the inclusion. When using finite elements to analyze unit cells, axisymmetric models are usually favored, as they reduce complexity by eliminating the need to perform a full $3 \mathrm{D}$ analysis ${ }^{[3,5]}$. 
The assemblage model is another analytical model which assumes an ideal reinforcement distribution, with inclusions in the form of spheres or cylinders. The microstructure is assumed to be constructed out of several spheres or cylinders which have reinforcing particles embedded within them. The local volume fraction in each sphere/cylinder is equal to the macroscopic volume fraction. The analytical solution obtained using this model gives limits for the overall properties of the composite, which are similar to the H-S bounds.

\subsubsection{Approximate Models}

This is a broad category of models comprising various methods to provide approximate solutions to the overall response of the composite. Though several researchers have contributed extensively to the development of approximate models, the principal models are: Self-consistent model ${ }^{[33]}$, differential self-consistent model ${ }^{[34]}$, generalized self-consistent model ${ }^{[35]}$, dilute approximation model ${ }^{[36]}$, Mori-Tanaka method ${ }^{[37]}$. All the models in this class contend that the inclusion strain is related to the overall elastic strain through a strain concentration tensor and hence the strain concentration tensor must be used in the estimation of the composite modulus ${ }^{[9]}$.

The self-consistent model estimates the strain concentration tensor by assuming that the inclusion particle in the form of an ellipsoid is embedded in the composite material whose properties are being estimated. This leads to the solution where the composite modulus is obtained by solving the following equations:

$$
\begin{gathered}
\frac{V_{r}}{K_{c}-K_{m}}+\frac{V_{m}}{K_{c}-K_{r}}=\frac{\alpha_{c}}{K_{c}} \\
\frac{V_{r}}{G_{c}-G_{m}}+\frac{V_{m}}{G_{c}-G_{r}}=\frac{\beta_{c}}{G_{c}}
\end{gathered}
$$

where $\alpha$ and $\beta$ are functions of the Poisson's ratio of the overall composite.

In the differential self-consistent model ${ }^{[34]}$, a spherical inclusion is assumed to be embedded in the composite, which has an inclusion volume fraction $\mathrm{V}_{\mathrm{r}}$. This leads to a 
set of differential equations which is solved with the initial conditions being assumed as $\mathrm{V}_{\mathrm{r}}=0$ at $\mathrm{t}=0$ and $\mathrm{K}_{\mathrm{c}}=\mathrm{K}_{\mathrm{m}}, \mathrm{G}_{\mathrm{c}}=\mathrm{G}_{\mathrm{m}}$ respectively.

The difference between the self-consistent model and the generalized selfconsistent model is that in the latter, the inclusion is surrounded first by the matrix material, which is, in turn, embedded in the overall composite material. The model is so constructed as to match the local reinforcement fraction with the overall volume fraction of the composite. This leads to a solution whose bulk modulus is the same as the H-S lower bound. The shear modulus is obtained by solving the equation:

$$
A\left(\frac{G_{c}}{G_{m}}\right)^{2}+2 B\left(\frac{G_{c}}{G_{m}}\right)+C=0
$$

where $\mathrm{A}, \mathrm{B}$ and $\mathrm{C}$ are constants.

In the dilute approximation model ${ }^{[36]}$, the inclusions are treated as ellipsoidal particles and only one particle is considered, thus neglecting inter-particle effects on the overall response of the composite. The Mori-Tanaka model ${ }^{[37]}$ does not provide any physical interpretation, but uses the eigen-strains in spherical reinforcing particles to estimate the average overall composite stress. The predictions from this model are close to the values from the generalized self-consistent model.

Due to the inherent complexity in deriving closed-form solutions from analytical models, computational methods like finite element (FE) models of unit cells have been preferred in recent years. FE models also provide microscopic stress and strain distributions, which are not available in most analytical models. Additionally, due to the increase in computational power available to researchers, more complex and more detailed FE models of microstructures have been developed ${ }^{[1,2,7]}$. Unlike traditional efforts, which depended on axisymmetric analysis of unit cells, it has now become feasible to conduct complete 3D FE investigations using models which contain realistic MMC microstructure information.

The current study aims at providing a computationally efficient technique, which can be used to build FE models that simulate the real original microstructure. 


\subsection{Problem Statement}

Two and three dimensional unit cell models being used today assume ideal microstructural conditions and employ unrealistic boundary conditions. While they may serve to reasonably predict the properties of fiber reinforced composites, the highly complex 3D microstructures of PRMMCs cannot be described using such models. To completely quantify microstructures of PRMMCs, the model has to contain information about the size, distribution, orientation and aspect ratio of the inclusions as well as other microstructural features such as particle clustering, at different modeling scales. Also, it is desired to develop a model that is easy to generate and computationally inexpensive.

The effort, to create a multi-scale modeling technique which predicts the microscopic as well as the overall response, of particulate reinforced composites is undertaken here.

\subsection{Research Objectives}

A central objective of this study is to characterize Aluminum 6061 reinforced with silicon carbide $(\mathrm{SiC})$ particles at various volume fractions, which is produced commercially by the DURALCAN Corporation by using a low-cost stir-casting technique. For this purpose a new unit cell model, which contains randomly distributed inclusion particles, in the form of spheres or ellipsoids is developed. To account for higher-scale effects such as clustering, information from the micro-scale model is input into a meso-scale model. Finally, the material response as predicted by the micro and meso-scale models is used in a macro-scale FE problem of practical significance to MMCs, namely the modeling and analysis of bolted joint connections.

In each stage of the study, the predictions of the FE model are validated through correlations with, values from experiments conducted by the author, or values from the literature. ANSYS and LS-DYNA are the commercial FEA software used in the study. The algorithms used to generate the artificial microstructure are implemented in the MATLAB computing environment. 


\subsection{Organization of the thesis}

1. In the next chapter, an extensive literature review is presented about previous attempts at modeling PRMMCs. The literature review also covers earlier modeling and testing of bolted joints.

2. In Chapter 3, three FE models are generated to represent the microstructure of the $\mathrm{Al} / \mathrm{SiC}$ composite. Two of these models involve iterative procedures whose algorithms are explained in detail. Furthermore, boundary conditions and loads are defined before the models are solved.

3. The results from all the three FE models are compared with each other and with experimental values. Microscopic responses of the composite are discussed in this chapter

4. In Chapter 5, the generation of a mesoscale model and a macroscopic model of a bolted joint are discussed.

5. The testing of bolted joints is discussed in Chapter 6 , along with the validation of the FE model, by comparing its predictions with experimental values.

6. Chapter 7 presents a summary of conclusions drawn from the study, along with suggestions for future work. 


\section{Chapter Two}

\section{Literature review of microstructure modeling and testing of metal matrix composites}

\subsection{Microstructure Modeling}

Metal matrix composites (MMC) have been extensively studied for the past decade in order to understand the effect of various microstructural parameters on their properties. Many attempts have been made at representing the microstructure of the MMC in a computational model in order to predict their properties. The simplest finite element representation of a discontinuously reinforced material is the unit cell model (UCM). A UCM treats the material as a periodic arrangement of RVEs, each having a single reinforcement particle embedded in the matrix. The RVEs may be square shaped for a 2-dimensional model or a cube for a 3D model and the inclusions are generally approximated as circles or spheres. The main drawbacks of the UCMs are that they fix the inter-particle distances and that the predicted stress distribution does not take into consideration the effect of neighboring particles.

\subsubsection{Unit Cell Models}

Taggard and Qin ${ }^{[9]}$ reviewed a comprehensive set of classical analytical and numerical models used to predict the elastic and elastic-plastic response of particle and whisker reinforced composites. They provided a comparison between all the analytical methods and numerical modeling techniques in use today and concluded that the MoriTanaka ${ }^{[10]}$ model was best suited for predicting the overall elastic-plastic response of the material and that the periodic array model had to be used to obtain microscopic stress information. The effect of inclusion aspect ratio on the microscopic stresses was also illustrated.

Bruzzi et al ${ }^{[1]}$ developed a periodic unit cell model for the numerical prediction of material properties of $\mathrm{Al}-2124 / \mathrm{SiC} \mathrm{MMC}$ with $17 \%$ reinforcement volume fraction. 
Initially, 2D plain strain finite element models with idealized inclusion shaped were developed. This model was upgraded to include more realistic shapes of reinforcing particles, which were obtained from actual scanning electron microscopy (SEM) images of the material. A 3D model having multiple particles, which includes residual stresses in the material due to the thermal expansion mismatch of the constituents, was the most realistic model analyzed.

Initially, the material was assumed to be an infinite periodic array of unit cells, with ideal bonding between the inclusion and the matrix. Simple symmetry boundary conditions were assumed for the $2 \mathrm{D}$ and $3 \mathrm{D}$ models such that they remain square and orthorhombic respectively. Loading was applied on the remaining surfaces in the form of uniform displacement conditions. 4 noded plain strain elements were used to mesh the matrix while plain strain elements having 3 or 6 nodes were used to mesh the inclusions in the 2D model. 8 noded linear or 20 noded hexahedron elements were used to mesh the 3D model.

A simple iterative embedding technique as an alternative to periodic boundary conditions was implemented. A unit cell with distinct inclusion and matrix phases was embedded in a homogeneous material having the overall composite properties. Boundary conditions and loading were applied on the homogeneous material. This configuration allowed realistic deformation of the unit cell such as rotation of the unit cell boundaries and non-uniform deformation of the cell boundaries.

All the models having idealized particle shapes reasonably predicted the MMC modulus but underestimated the MMC strength. In models having realistic particle geometries, it was seen that higher plastic strain was recorded when inclusions were closer together and particle distribution was non-uniform. The results showed that models having realistic particle geometries made a more accurate prediction than models considering ideal particle geometries. The same was true for the 3D models.

The embedded unit cell model predicted that the cell boundaries do not remain straight after loading, but since the difference in the value of flow stress levels obtained by the two approaches was less than $3 \%$, it was concluded that the periodic cell approach predicted the overall behavior with sufficient accuracy using much less computational resources. 
Another criticism of the periodic array model is that the particles are idealized as spheres or cubes. To overcome this simplification, Shen et al ${ }^{[2]}$ used 2D and 3D UCMs having single and multiple particles to study the deformation in MMCs. A 24-faced solid was used to approximate the shape of the inclusion. The model assumes elastic particles and an elasto-plastic matrix with linear hardening used for the plastic region of the matrix material. Internal thermal stresses and damage progression were ignored. Volumeweighted averages were used to calculate the overall stress and strain in the cubic domain. The loading was assumed to be at room temperature and particle cracking was ignored.

A 3D unit cell model was created for the sake of comparison with the 3D multiparticle model. The 3D unit cell (UC) was meshed using 10 node tetrahedral elements and symmetry conditions were applied on 3 of its coordinate directions. A 3D multiparticle model was created with each side of the representative volume element (RVE) at $50 \mu \mathrm{m}$. The centers of the inclusions were determined using a random number generator based on inverse cumulative distribution function method ${ }^{[38]}$. Along with the $3 \mathrm{D}$ models, several 2D FE models were generated which had area fractions of $9 \% .17 .5 \%$ and $26 \%$. 6 noded triangular elements were used to mesh these 2D models. Generalized plane strain was assumed and it was found that the results from the $2 \mathrm{D}$ models were in close agreement with the values from the 3D model.

The values of stiffness and flow stress from the 2D model varied with the area fraction of the inclusion. Convergence of the solution was checked by measuring the effective plastic stress at a particular point and by comparing the change in the plastic strain from a series of fine meshes. It was also concluded that while a clear plastic strain distribution can be seen in 2D models, such a distribution is not visible in 3D models. They also confirmed that the maximum tri-axial stresses are induced between particles in the loading direction. Significant differences were observed between the predictions of 2D and 3D models regarding the distribution of principal stresses and plastic strains.

Huang and Bush ${ }^{[3]}$ studied the elastic and plastic properties of alumina/aluminum MMC having an ultra-fine microstructure by using FE models. They investigated the 
effect of grain size of the matrix material on the mechanical properties of the material. The finite element model incorporated a weak phase to represent the porosity of the material, which is inherent to MMCs produced by powder metallurgy method ${ }^{[39]}$. The first part of the study concentrated on predicting the elastic modulus of the MMC using a unit cell model and by comparing this with published values, predicts an effective modulus for the weak phase. In the second part, to study the plastic properties of the material, an indentation process was simulated and its results compared with experimental values to gain an insight into the properties of ultra-fine grained MMCs.

The MMC specimens required for experimental evaluation $(0 \%, 10 \%, 20 \%$ and $30 \%$ ) were prepared by powder metallurgy process. The vacuum dried alumina and aluminum powders were compacted in a sealed die using a $64 \mathrm{~kg}$ mass falling from 4.5 meters. The compacted specimens were subjected to sintering at $600{ }^{\circ} \mathrm{C}$ for one hour. The specimens, which were $35 \mathrm{~mm}$ in diameter and $5 \mathrm{~mm}$. thick, were polished using diamond plates and hardness tests were carried out. The grain size of the matrix was measured by using the X-ray diffraction spectra with the Williamson-Hall method ${ }^{[4]}$.

The FE model consisted of repeated unit cells with hexagonal arrangement, which incorporated porosity by use of a weak phase. Perfect bonding was assumed between the particle and the matrix. The model was meshed with 4 noded quadrilateral elements and loading was carried out by imposing uniform displacement condition on the top row of nodes. A series of models with successively finer meshes were produced to check for adequacy of finite element discretization. The indentation model consists of a circular indenter and cylindrical composite specimen. The contact between the indenter and the specimen was addressed as contact between two closely spaced deformable bodies capable of undergoing small sliding relative to each other.

The comparison between the experimental hardness testing and the FE indentation model showed a satisfactory agreement. The elastic properties predicted by the FE model were found to be slightly higher than the experimentally found values. The experimental values were also found to stray beyond the Hashin-Shtrikman bounds. Porosity was cited as the reason for this lower value. FE analysis of a model which incorporated the weak porous phase confirmed this contention. A reasonable agreement between the experimental values and indentation model were seen for $10 \%$ volume fraction, but 
deviations were found for $20 \%$ and $30 \%$ volume fraction. A high dislocation density and the pattern of reinforcement distribution were cited as reasons for this behavior.

Leggoe et al ${ }^{[5]}$ used a two-scale finite element approach to study deformation in PRMMCs. Axisymmetric unit cell models were used to predict the mesoscale properties of composites, while macroscale properties of the composite were predicted using a random array of such finite elements. Due to the highly three-dimensional microstructure of PRMMCs, a 3D unit cell model was developed in the first stage to accurately predict the properties of the PRMMC. A family of constitutive properties on the mesoscale was generated for a wide range of volume fractions (5\%-40\%). To determine the macroscale response, a solid member was meshed with each element being treated as an isotropic, homogeneous region. The properties of each element were randomly assigned in accordance with a governing statistical distribution - as having $0 \%$ to $40 \%$ volume fraction, thus producing a random finite element array. Random arrays in 2 and 3 dimensions were produced with varying degree of heterogeneity to study its effect on the material response. For each reinforcement distribution a set of 10 random arrays were analyzed to quantify the variation in the predicted response.

The mesoscale response was generated by using axisymmetric unit cell models which had hexagonal prisms containing a single spherical inclusion at its center. The mesh was generated using isoparametric 4 node quadratic elements. The inclusion was assumed to be perfectly elastic, while a bilinear approximation was used for the matrix. The usual symmetry and uniform displacement boundary conditions were imposed on the 4 faces and the model was loaded by applying an incremental displacement on the top surface

In the macro-scale model, zero displacement boundary condition was imposed on the faces intersecting at the origin, while the other three faces had the same displacement on all points. The random array was loaded by imposing incremental displacement on the right hand face. Various random arrays with varying deviations in the volume fractions were analyzed to study the material behavior.

It was found that the axisymmetric models predicted the elastic modulus between the Hashin-Strikman range and that the model displayed pseudo-strain hardening. It was found that the effect of boundary conditions on the material response was negligible for 
low volume fractions but was significant at higher volume fractions. For the macro-scale model, the plastic strain accumulated at regions which had un-reinforced elements. With increase in loading, these zones of high strain joined to form bands and the high stresses at these bands promoted yielding. The array size had little effect on the predicted elastic modulus, but the peak stress decreased with increase in the array size. The predicted responses were in-line with the previously obtained results from $2 \mathrm{D}$ models of heterogeneous materials.

Another comprehensive review of the current state of the art in the computational mechanics was carried out by Schmauder ${ }^{[13]}$. He concentrated especially on numerical homogenization techniques for simulation of real microstructural behavior of MMCs reinforced by continuous fibers and discrete particles. Special emphasis was laid on the embedded cell technique and a comparison between the results of UCMs and embedded cell models (ECM) was carried out. The ECM contains a single spherical inclusion surrounded by the matrix, which is in turn embedded in an equivalent composite material. The advantage of this kind of model is that the symmetry boundary conditions are not applied directly on the constituent materials, but on the surrounding equivalent material. The properties of the surrounding material are found in an iterative manner with its properties being updated with the results from the inner cell for each iteration, until they converge to an invariant value. 2-D and axisymmetric 3D models were analyzed for various shapes of embedded cells and it was found that the shape of the embedded cell has little effect on the overall response.

The results from the ECM agreed very well with the experimental results and less with the UCM results. The strength of the composite as predicted by different models was compared and an equation for predicting the strengthening of the composite, given the matrix yield stress, matrix strain hardening component and the reinforcement volume fraction was proposed.

$$
\bar{\sigma}_{N}=\sigma_{0}\left[\left(1-\frac{f}{c_{1}(2+N)}\right)^{-\left(c_{2} N+c_{3}\right)}-c_{4}\left(f+\frac{N}{5}\right)\right]
$$


where $\mathrm{c}_{1}, \mathrm{c}_{2}, \mathrm{c}_{3}, \mathrm{c}_{4}$ are constants.

Baptiste $^{[8]}$ included the effects of plasticity and damage in the prediction of mechanical properties of particle reinforced metal matrix composites. He provides a homogenization frame work in which damage can be included in the micromechanical response of the material through the use of Weibull Law ${ }^{[11]}$ to account for fractured reinforcement particles. The Weibull law gives probability that a particle has fractured dependent on the size of the inclusion and maximum principal stress it experiences. Though a broken particle continues to contribute to the overall stiffness of the composite, they also give rise to cracks. A criterion which has a linear combination of interfacial normal and shear stress is used to determine whether de-bonding has occurred or not and accordingly, the effective stiffness of the composite is adjusted. The damage is modeled in the form of ellipsoidal cracks having a stiffness of zero and the anisotropy caused due to the accumulation of damage is also taken into consideration.

All of the computational models explained above suffer from same shortcomings that they idealize the microstructure of the MMC. The particles are idealized as spheres or ellipses. Further, a single particle is considered per unit cell, and even when multiparticle unit cells are produced, the inter-particle is held constant. This is not only an unrealistic assumption, but also results in the inclusion having unreasonable correlation lengths with respect to the unit cell. As a result, although unit cells are reasonably accurate in predicting the stiffness of MMCs, they vary widely in their strength predictions. Consequently, there has been an effort in recent years to develop FE models which incorporate real microstructures in them. Several strategies ranging from image analysis to fractal geometry have been used in this direction.

\subsubsection{Voronoi Cell Models}

One of the most widely known methods for the reconstruction of PRMMC microstructures is through the use of Voronoi cells. In a series of papers, Ghosh et. al ${ }^{[14-}$ 17] developed a method of microstructure reconstruction, which involved serial sectioning $^{[18]}$ of the material combined with Voronoi tessellation of the resulting 
microstructure representation. Serial sectioning is a technique which involves gradual removal of parallel layers of MMC in order to obtain several optical micrographs. These micrographs represent the 2D microstructure of the material at various depths. Figure 2.1 (a) shows such a 2D microstructure obtained from an optical micrograph. By stacking consecutive micrographs, a 3D representation of the microstructure is computationally generated. The critical parameter in this process is the depth of material removal for each micrograph, which depends on the amount of microstructural detail desired in the model. The digitized 2D images were stacked and assembled together using special software packages.

Due to the computational expense of analyzing microstructures with arbitrary particle shapes, the digitized particles were converted to ellipses for 2D microstructures and ellipsoids for 3D representations. This microstructure is further modified to eliminate inclusion impingement.

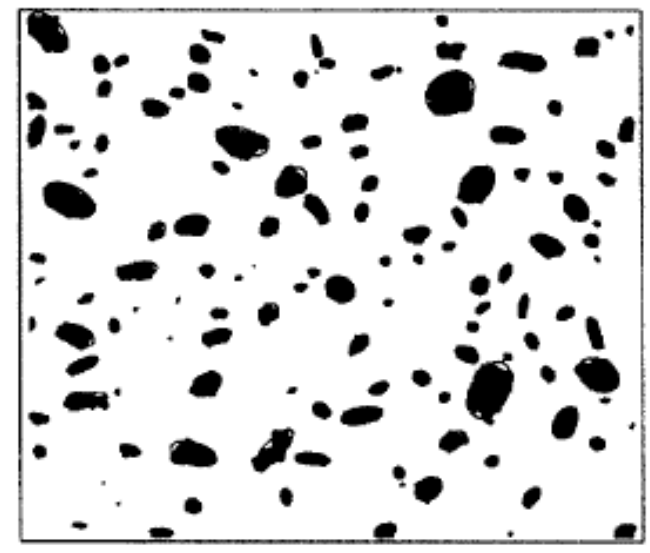

(a)

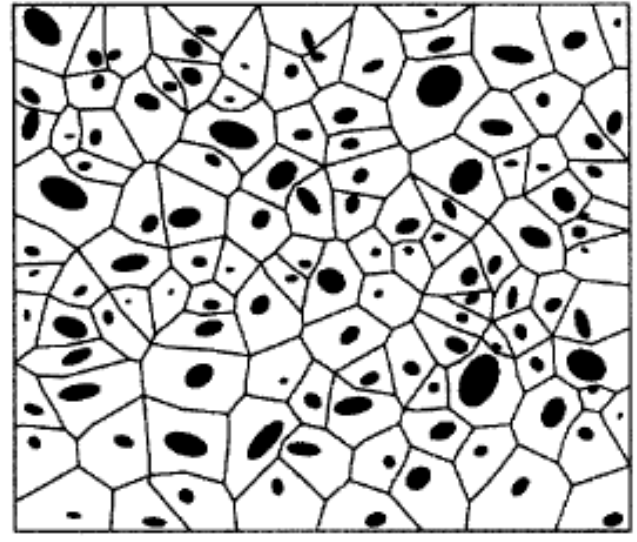

(b)

Figure 2.1 (a) Optical micrograph of a section having $10 \%$ inclusion fraction (b) Voronoi cell tessellation of the equivalent microstructure. ${ }^{[15]}$

This representative domain was discretized by Dirichlet tessellation ${ }^{[40]}$, producing Voronoi diagram partitions, which is a collection of convex polygons. Dirichlet tessellation of a matrix containing inclusions is defined as the subdivision of the domain such that each inclusion has a region associated with it, which is closer to it than any other particle. The Voronoi polygons produced in this manner were considered as elements in the finite element formulation. Thus, each element in the domain had a single 
reinforcing particle at its center. Fig 2.1 (b) shows a tessellated 2D microstructure with the particles approximated as ellipses.

A special finite element having arbitrary number of edges was formulated using the stress-hybrid finite element method ${ }^{[41]}$. The formulation assumed inter-element continuity in displacements and the stress was interpolated within the element using a function such that the equilibrium condition is satisfied. Additionally, traction and displacement continuity is enforced across the particle-matrix interface but the stresses may be discontinuous. Lastly, for each element, the rigid body modes of the particle/matrix interface are set equal to that of the element boundary.

Grujicic and Zhang ${ }^{[19]}$ used the Voronoi scheme to evaluate the elastic properties of a functionally graded material. A functionally graded material of $\mathrm{Ni} / \mathrm{MgO}$ was selected with the volume fraction varying from 0 to 0.3 . A $2 \mathrm{D}$ representation of the microstructure in a square domain was used for the analysis. The microstructure contained three types of inclusions - nickel, magnesium oxide and porosities. The microstructure was loaded in tension and the in-plane Young's modulus was calculated. A comparison of predictions with experimental values showed that the results were closer to experimental values than those predicted by the self-consistent method ${ }^{[33]}$ and equivalent inclusion method.

\subsubsection{Multi-scale Models}

A multi-scale model for damage prediction in fiber reinforced composites, developed by Raghavan et al. ${ }^{[20]}$ also made use of Voronoi elements. The problem selected for analysis was that of a bonded double lap joint containing boron-epoxy composite. The multi-scale model consisted of three levels - level 0 was the purely macroscopic level with homogenized material properties, level 1 was the meso-scale level used to signal if a switch to purely microscopic analysis was required in the region and level 2, which was a purely microscopic level was produced from the Dirichlet tessellation of optical micrographs of real microstructures.

Kenesei et al ${ }^{[6]}$ used holo-tomography to obtain a real 3D microstructure of the material which was used as input to the model. They started with the contention that 
particle arrangements in real materials are not random and so it is easier to model real microstructure instead of using computer generated microstructures. The tomography study was carried out at the European Synchrotron Radiation Facility on a commercially available MMC sample. The microstructure thus produced was correlated using a 2-point probability function $\mathrm{P}_{11}$, which is defined as the probability that two points separated by a distance $r$ and randomly chosen, both lie in the matrix phase. The contour of this $\mathrm{P}_{11}$ function is shown in Figure 2.2 (a) and (b) for cuts perpendicular and parallel to the extrusion axis. The correlation lengths are determined from this plot and suitable RVE dimensions are determined from these correlation lengths.

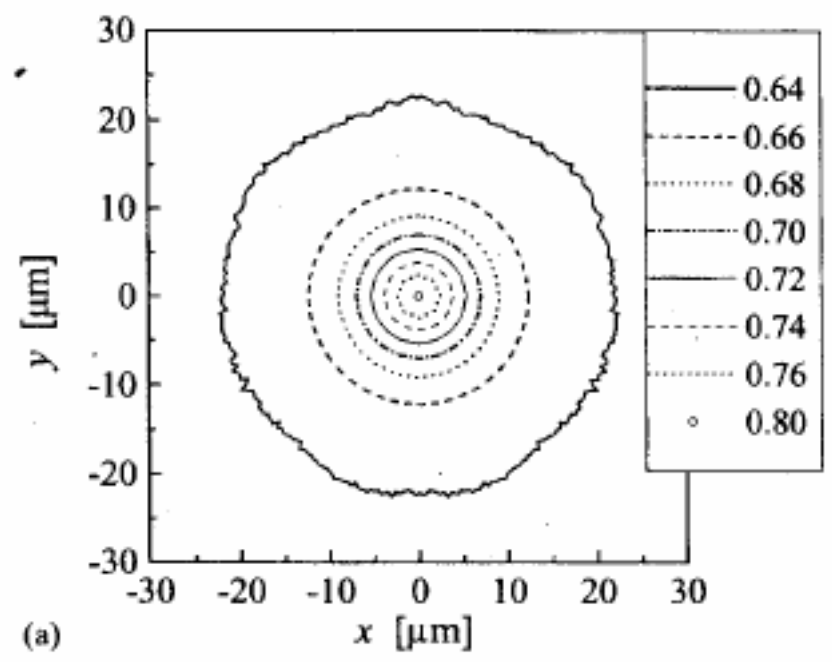

(a) along the extrusion direction

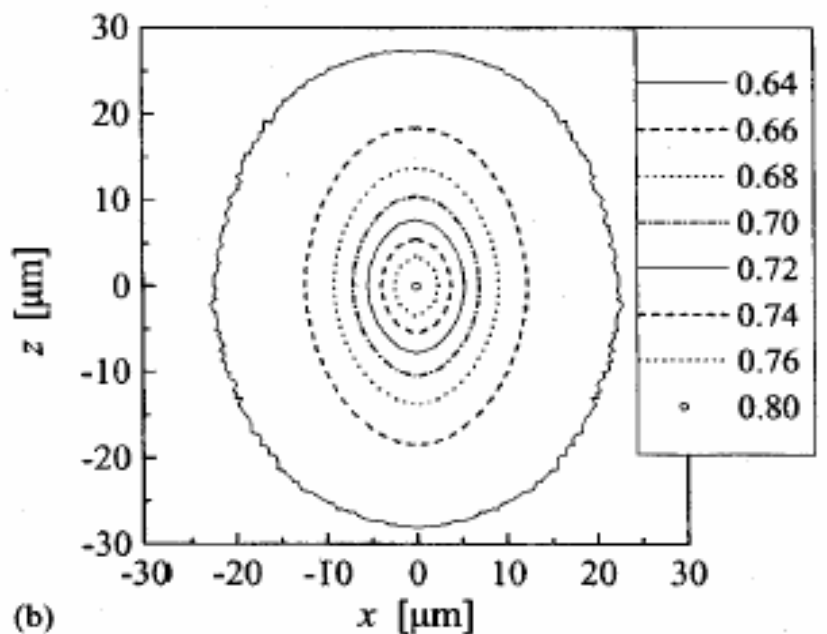

(b) perpendicular to the protrusion direction Figure 2.2- Plot of the $\mathbf{P}_{11}$ functions ${ }^{[6]}$

A cubical volume, which is assumed to be mechanically representative of the macroscopic material and having each side of length 9-10 times the correlation length was considered directly from the binarized tomographic reconstruction. Due to the huge number of elements in the model, it was further sub-divided into windows. Depending on the ceramic content of each of the window, either it was assigned particle or reinforcement properties. It was seen that a smaller averaging window size resulted in a more accurate representation of the microstructure and that larger window sizes resulted in concave particles. Compression tests which were simulated were compared with 
experimental compression tests. The FE modeling was done using MSC.MARC and damage was neglected during analysis. The meshing was performed using isoparametric elements with the tri-linear interpolating functions. Macroscopic properties of a given model were obtained by using volume weighted averages of element quantities.

The Young's modulus obtained from the model was found to decrease with the average window size. Using extrapolation on the data at hand reveled a modulus value of $103 \mathrm{GPa}$ and $98 \mathrm{GPa}$ in the extrusion direction and perpendicular to extrusion direction respectively. It was also seen that higher flow stress was induced in the direction of extrusion due to the alignment of the reinforcing particles, which have an aspect ratio of 4:3:2. The errors involved in the prediction of the plastic and elastic properties were estimated to be $4 \%$ and $10 \%$ respectively.

Though VCFEM and holotomography provide accurate representations of the microstructure, the process involved is either tedious or requires special hardware for implementation. One alternative to overcome these shortcomings is to artificially generate microstructures which incorporate most of the important characteristics of real microstructures. These methods often make use of computer generated microstructures which use statistical measures to correlate the generated microstructures to real microstructures.

A multi-inclusion unit cell model in three dimensions was proposed by Bohm et $a l^{[7]}$ as a means for predicting the thermo-mechanical properties of composites reinforced by nonaligned short-fibers. They used the sequential adsorption approach to generate microstructures containing cylindrical, spheroidal and spherical inclusions, as shown in Figure 2.3. This approach involves generating random inclusion positions and these positions are retained if they do not overlap any previously defined inclusions and are rejected otherwise. The technique was modified to accept a user-defined minimum distance between neighboring particles, which is helpful in maintaining uniform distribution of fiber orientations. Inclusions intersecting with one or more faces of the unit cell were split into appropriate number of parts in accordance with the periodicity. 


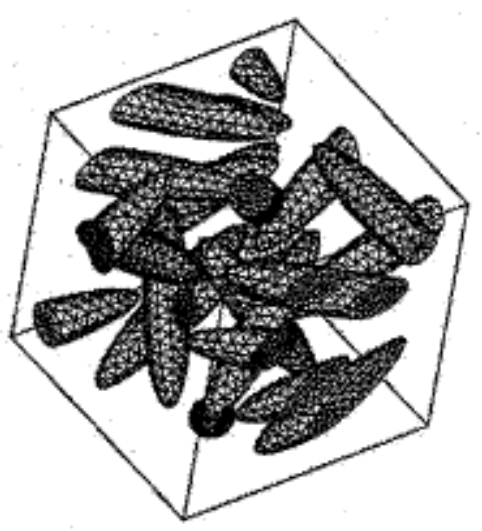

(a) Spheroidal fibers

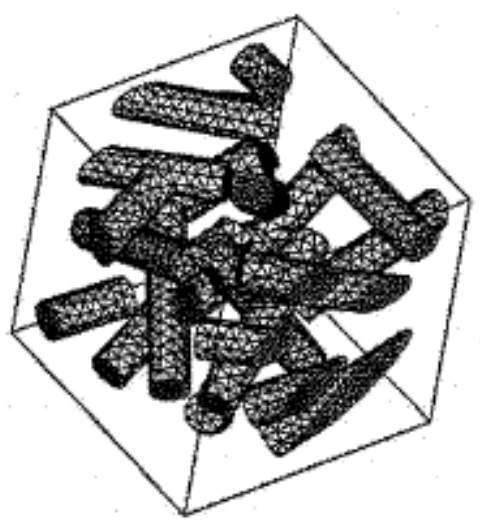

(b) Cylindrical fibers

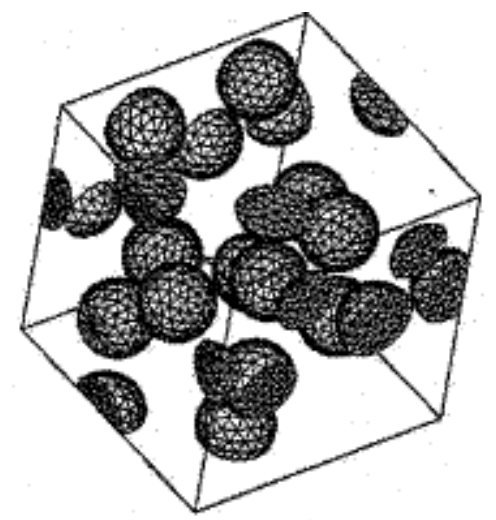

(c) Spheres

Figure 2.3 - Equivalent microstructures for inclusions ${ }^{[7]}$

The fibers were assumed to be made of silicon carbide and an elasto-plastic matrix of aluminum. A modified Ludwik strain-hardening law was applied on the matrix, according to which,

$$
\sigma_{y}=\sigma_{y, 0}+h\left(\varepsilon_{e q v, p}\right)^{n}
$$

where $\sigma_{\mathrm{y}}$ is the actual flow stress

$$
\sigma_{\mathrm{y}, 0} \text { is the yield stress }
$$

$\varepsilon_{\text {eqv,p }}$ is the accumulated elastic strain

$\mathrm{h}, \mathrm{n}$ are the hardening coefficient and the hardening exponent respectively.

The Young's modulus and Poisson's ratio predicted from all the models fell between the H-S bounds, though short fibers experienced more stresses than particle reinforcements. The model having spherical reinforcements displayed weaker strainhardening than the models with fiber reinforcements. The distribution of Weibull fracture probabilities showed that fiber direction plays a dominant role in failure characteristics of the composite. It was concluded that materials having inclusions in the form of short fibers have a higher tendency for reinforcement failure than particulate reinforced materials.

Cottet ${ }^{[21]}$ has attempted to represent the complex microstructure of ceramic matrix composites using fractal spatial particle distribution. Fractal geometry was made use of to describe the randomness and disorder inherent in inclusion distribution. An 
iterative algorithm was developed using the Iterated Functions Systems (IFS) theorem ${ }^{[42]}$. The goal was to generate different microstructures having the same volume fraction and surface area, but different fractal dimensions, with the intention of associating material properties to the fractal dimension of the microstructure.

2D Finite element analysis of the obtained microstructures showed that particle clustering played an important part in the overall properties of the material. Toughness depended on the local distribution of particles and their sizes. It was also shown that the microstructures having the highest fractal dimensions give the best results. Along with fractal dimensions, other characteristics of the microstructure such as lacunarity was required to effective characterize the microstructure.

The problem of damage evolution and failure in fiber reinforced MMCs was addressed by Xia et al ${ }^{[22]}$. A multi-scale approach was used to study the distribution of stresses in a yielding titanium matrix around failed silicon carbide fibers. The FE model was built from a hexagonal array of fibers with the central fiber as the broken fiber. The fiber-matrix interface was defined with various coefficients of friction and the distribution of shear stresses around the broken fiber showed a greater variation for higher coefficients of friction. As the friction coefficient increases, the neighboring fibers bear a higher percentage of load from the broken fiber. This level of modeling determines the amount of load transfer between the fiber and matrix.

The Green's function method was used to predict the stress distribution due to multiple fibers and it uses two factors - the radial stress concentration due to the broken fiber and stress concentration along the fiber. These two factors are obtained from the FE simulations performed in the previous step. To test the accuracy of this analytical method, a separate FE model having 7 broken fibers was built and the predictions agreed very well with each other. Finally, a comparison between strength values obtained from experimental testing and numerical model for various values of frictional coefficients showed that they are in excellent agreement. 


\subsubsection{Summary of Micromechanical Models}

As can be seen from the above review, though significant amount of work has been done on predicting the behavior of continuously reinforced MMCs, there is an absence of 3D microstructure models for PRMMCs which reasonably mimic real microstructures and are computationally efficient at the same time. The current study attempts to fill this gap by analyzing a computer generated artificial microstructure having most of the characteristics of a real PRMMC microstructure.

\subsection{FE modeling and testing of bolted joints}

In a multi-scale modeling approach, the properties obtained from micro/meso scale models are inculcated in macroscale models to determine the response of a real life structure made of that material. In the current study, bolted joints made of $\mathrm{Al} / \mathrm{SiC} \mathrm{MMC}$ material were chosen as the structure of choice as the ability to use bolted joint connections is viewed as one of the major advantage with PRMMC material.

For many years now, bolted joints made of different materials have been analyzed all over the world and standards have been developed which govern the design of such joints. Ramadan et al ${ }^{[23]}$ investigated fin plate connections (bolted joints used to transfer loads between simply supported beams and supporting members) using both experiments and FE models. For experiments, a test rig was designed which could measure the effects of various factors like number of bolts, plate thickness and the angle of load application. One, two and three-bolt connections were tested under load angles between $0^{\circ}$ and $90^{\circ}$, both under tension and compression.

The finite element model constructed in ANSYS 5.1 contained the web, flange of the beam and shank of the bolt, which were made of eight-node isoparametric solid elements. The interface between the bolt and the plate was modeled using 5-node pyramid contact elements. Though bolt heads and nuts were not modeled, their effects were accounted for by including equivalent axial forces and coupling the degrees of freedom of concerned nodes. A multi-linear material model for the beam members and tri-linear approximation for the bolt material was used. 
From the experiments, it was clear that specimen yield at the contact between the bolt and the plate and that the stiffness of the joint after yielding was dependent on the loading angle. The post-yield stiffness increased with increase in loading angle and the difference in stiffness before and after yielding increased with increase in bolt diameter. The maximum load carrying capacity decreased with increase in loading angle. The results from the finite element program correlated well with the experimental values.

The effect of bolt clearance in a single shear composite joint having a [45/0/$45 / 90]_{S}$ layup was investigated by Lowlor et al ${ }^{[31]}$. The clearance in the joint was altered by having a constant bolt diameter and varying the hole radius. The load-displacement curves for finger-tight condition showed that the stiffness of the joint decreased with increase in clearance, but the maximum load of the joint remained nearly constant. A similar trend was also seen in joints torqued to a higher degree. The bearing stress and strain was calculated from the load-displacement data obtained above and it was seen that for joints having protruding head bolts, the effect of clearance on the ultimate strength was negligible. But increasing the clearance between the bolt and plate did increase the ultimate strain to a large extent.

Laviolette et al ${ }^{[24]}$ also used the approach of experimental and FE modeling to study bolted joints in pipe flange connections under combined bending and internal pressure. The FE model consisted of radial loads represented as a superimposition of 7 different loads around the circumference of the pipe and bending loads. The model was constructed using axisymmetric shell elements. A test rig which used transducers to record the contact stresses in the gasket was setup measure the leakage behavior of the gasket with increase in bending load.

The results showed that the bolt load decreased with increase in the load cycle and this agreed very well with the FE simulations. The stress distribution across the width of the gasket as predicted by the FE model varied with the experimental values due to the change in the Young's modulus in the gasket material at high compression. It was concluded that though the American Society of Mechanical Engineers (ASME) 
specification is adequate from a structural point of view, it may not be conservative for joint leakage behavior.

Another study regarding bolted flange connections was carried out by Lin et al ${ }^{[25]}$ by using only finite element analysis. Their model consisted of two flanges connected together by a set of 14 bolts. Utilizing the symmetry of the problem, a pie-shaped piece of flange containing one bolt-hole, shown in Figure 2.4, was modeled. The bolt head was accounted for in the form of a thick washer, which was assigned a Young's modulus 1000 times that of the flange material. Various models were generated, having different bolt diameter-to-length ratios.

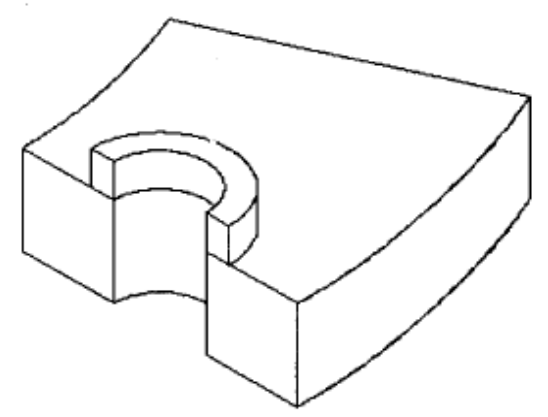

Figure 2.4 - The part of the bolted flange modeled using finite elements. ${ }^{[25]}$

The two radially extending planes were constrained, along with the bottom surface. The rest of the surfaces were free to deform and load was applied in the form of a pressure to the top surface of the washer. The stiffness of the joint was calculated as the load per unit displacement of the washer. It was seen that the variation of the stiffness of the joint with the aspect ratio of the bolt can be approximated using a bi-linear fit. Also, this stiffness is dependent on the Poisson's ratio of the material. The 3D model generated in Lin's study predicted a higher stiffness as compared to the previous 2D models.

One of the few studies that dealt with bolted connections of MMC plates was conducted by Soni et al ${ }^{[26]}$. A combined numerical, analytical and experimental approach was taken in this study. The material used in the joint had a silicon matrix reinforced with titanium fibers in a quasi-isotropic layup of $[0 /+45 /-45 / 90]_{\mathrm{S}}$ having a volume fraction of $33 \%$. The experimental plan included testing the specimen at room temperature and at 
$650^{\circ} \mathrm{C}$. Four strain gauges were used on each specimen tested at room temperature and an extensometer was used to measure the strains in the high temperature specimen. Also, specimens coated with a polyester film were tested to measure its effect on the response of the joint.

Though the polyester film did not affect the failure load of the joint to a great extent, it changed the failure mode of the joint by redistributing the stresses after first ply failure. It also increased the strain capacity of the joint to a great extent. The strengths of the joints tested at $650^{\circ} \mathrm{C}$ were only $50 \%$ of the joints at room temperature, though the mode of failure remains the same - net tension.

Soni used mechanical properties obtained from an analytical model as inputs to the FE model. An in-house software tool, based on the concentric cylinders model was used to determine the constitutive properties of the composite. The model represents the composite as a set of 6 concentric cylinders with the matrix and fiber representing the innermost and outermost cylinders and 4 more cylinders of equal thickness used to denote the interfacial region. The finite element model consisted of one half of the joint modeled with 3D solid elements. The pin through the hole in the joint is treated as rigid and the interface is represented through contact elements.

Both the composite and the polyester film were approximated using bilinear material models. The results from the FE model agreed very well with the experimental values at room temperature, though they over-predicted the stiffness and strength by a small amount. The FE model did not take into consideration the effects of first ply failure and so could not predict the overall strength of the composite. Similarly, the low-strain predictions of the model at $650^{\circ} \mathrm{C}$ were close to the expected value.

Pratt and Pardoen ${ }^{[27]}$ conducted a study on joints having countersunk bolt-heads and showed that the MIL-HDBK-5 underestimates the strength of a single lap joint by up to $17 \%$. The military standard consists of compiling single bolt joint data by halving the results from dual-bolt joint data. This was shown to be a conservative estimate by conducting tests on single and double bolted joints combined with FE analysis. 


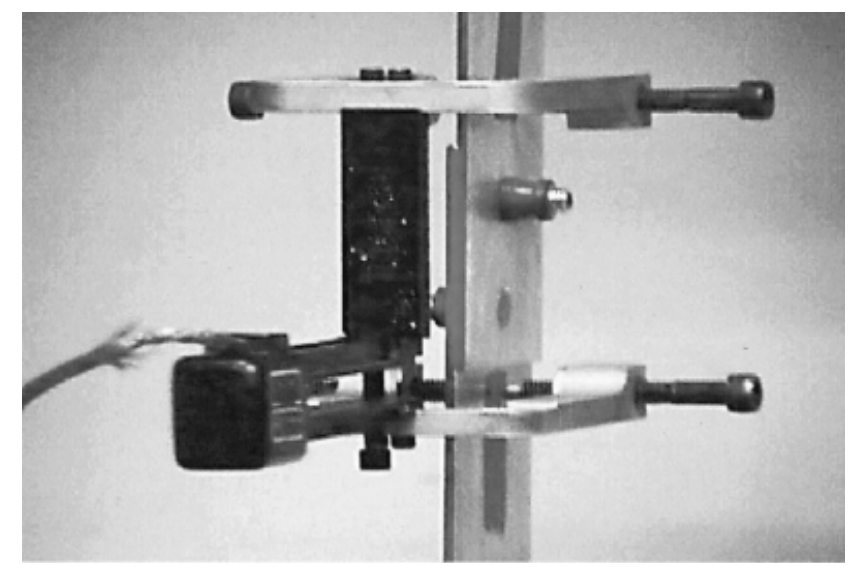

Figure 25 - Experimental setup with extensometer for testing single-shear bolted joints ${ }^{[27]}$

Extensometers, as shown in Figure 2.5, were attached to the tested specimens to obtain the load-elongation curves. The FE analysis was conducted using the NIKE3D code, along with the INGRID pre-processor and TAURUS post-processor. For efficiency, only the gage length of the joint was modeled and the interface between the fastener and panels was represented through tied-nodes. The nut and bolt of the joint were treated as elastic-plastic materials with bi-linear approximation, while the panels were treated as elastic-plastic material which followed the power-law hardening. The model was meshed using 8-noded isoparametric brick elements. The frictional coefficient for bolt-to-panel interface was set at 0.07 which was arrived at by trial and error through analyzing various joints with different configurations. A clamp load of 2500-4000 N was applied on the joint using a pre-strain of $2 \%$.

On comparing the experimental and numerical results, a high correlation between the two was seen. In thick panels, halving the double fastener joint strength values (which is the procedure followed by the Military handbook) resulted in a $10 \%$ underestimation of values. The difference between the two for thin panels was negligible. Similarly, an underestimation of strength by $17 \%$ was predicted by numerical models for thick panels, which correlates well with the experimental results.

An investigation into the structural behavior and failure modes of high strength, low ductility cold formed steel - which are somewhat comparable to $\mathrm{Al} / \mathrm{SiC} \mathrm{MMC}$ bolted joints was conducted by Chung and I ${ }^{[28]}$. The Fe model built in ANSYS used 8- 
noded brick elements for the joint and incorporated such features as clamping force, frictional resistance between plates, washer effects etc. The stress-strain curve obtained from coupon tests was input to the model for material properties. Strength degradation was adopted at higher strain levels to account fracture of steel at contact points having high localized stresses.

It was seen that the FE model predict the behavior of the connection accurately not only at low but also at high strain levels. It was also shown that the strength coefficient of high-strength but low ductility steel joints was lower than those of low strength, high ductility joints due to formation of highly localized yield zones in the former. Further, a parametric study with the FE model was conducted and the results were compared with the design codes recommended by various standards. The values from the design rules and FE model varied over a large range of values which was explained as being due to the variation in the frictional resistances in the joints, dependence on deformation limits by design rules and variations in steel strengths. Finally, the study proposed a semi-empirical design rule obtained from the calibrated FE model.

Another study on the behavior of bolted connections was conducted by Ju et al ${ }^{[29]}$ to study the plastic strain fields near the bolt and the crack behavior of steel plate along with the nominal applied force of the bolted connection. The joint configuration studied contained three rectangular plates with 1-3 bolted joints. The analysis accounted for the bolt clearance, washer, bolt head, gripping force, friction, and deformation of the bolt. A specially developed node-to-Hermite surface contact elements were used to model the contact between the bolt and the plates.

One half three plate connection was modeled with the bottom surface of the middle plate being constrained. The bottom surface of the bolt is also constrained to eliminate rotation of the bolt. Bilinear approximation was used for the material model of the steel plate along with the Von-Mises criterion to determine yielding. The results from the analysis showed that though the strain patterns were complicated, the capacity of the joint was within the prescribed AISC specification. Joints having bolt pre-tension and large plate thicknesses tended to fail by bolt failure rather than plate failure and three-bolt 
connections with thin plate thicknesses failed by net-section failure. It was observed that for a bolted joint having a crack in the plate, the stress intensity factor varies linearly with the applied load on the joint.

Bursi and Jaspart ${ }^{[30]}$ used a different kind of FE model combined with experimental testing to study beam-to-column joints. To acquire experimental information, a special test-rig combining a load cell, screw jack for load application and measurement and an LVDT to measure deflection was setup. The values from the experiments were compared with those obtained from EUROCODE 3 specifications and it was found that the code underestimates the stiffness and strength of the connection. Finite element models from the general purpose package ABAQUS was calibrated by using test data and an elementary tee-stub connection (proposed as a benchmark connection) is simulated. A new bolt model termed spin, which is a simplified representation of the bolt and the shank through beam elements, was validated. Lastly, simulations of end-plate connected joints were performed to assess the accuracy of finite element models.

\subsection{Summary}

Though several researchers have studied bolted joints in detail, there is little information available on bolted joints constructed from PRMMCs. Since MMCs are expected to be joined in this manner frequently, it is necessary that a parametric study be done on the effects of various parameters on the joint response. 


\section{Chapter Three}

\section{Finite Element Modeling of Microstructures}

\subsection{Introduction}

It is clear from the literature reviewed in the previous chapter that most past attempts at predicting the behavior of PRMMCs were based on generating different kinds of unit cell models having idealized particle shapes and distributions. Alternatives to UCMs, which aim to capture the complex distribution of particles in PRMMCs are either too time-consuming to generate or demand special hardware/software requirements. A second alternative, which has been explored to a lesser degree by past researchers, involves computer-generated microstructures to be analyzed using finite elements. This is the method of choice in this study.

Unlike UCMs, it is possible to generate artificial microstructures that do not place unrealistic restrictions on the distribution and shape of particles. Routines can be written to generate microstructures which resemble the actual microstructure is most respects and their correlation can be quantified by using statistical metrics such as average particle size, particle aspect ratio and its distribution, particle size distribution, nearest neighbor distance and its distribution etc.

One of the main criticisms against computer generated models is that high volume fractions cannot be generated by this method. Though this is true for very high volumefraction materials (excess of $40 \%$ ), most MMCs used in the industry today have a reinforcement volume fractions between 15\%-30\%. It will be shown in this study that even with relatively simple algorithms; it is possible to write efficient subroutines which easily achieve this level of volume-fractions. In the current study, artificial microstructure is created by locating the inclusions at randomly generated points. It has been suggested before that the location of inclusions is not completely random and neither is the orientation of inclusions. But this is highly dependent on the processing method used to produce the MMC. Processes such as powder extrusion ${ }^{[43]}$, powder rolling ${ }^{[44]}$ etc may result in orientation of inclusion particles in a few favorable directions, but the MMC 
used in the current study was produced using liquid-state stir casting ${ }^{[45]}$, which is devoid of such effects. Thus it is estimated that uniformly distributed inclusions can be used to represent the microstructure of the material at hand.

Three types of FE models were developed in the current study:

1. A body- centered cubic unit cell model.

2. A unit cell containing sphere-shaped inclusions at random locations.

3. A unit cell containing ellipsoid-shaped inclusions at random locations.

\subsection{Body centered cubic UCM}

The body-centered cubic UCM was developed for the sake of comparison with the newly developed random inclusion models. The BCC UCM is cube shaped with $1 / 8^{\text {th }}$ of a spherical inclusion embedded at each corner. A complete sphere is placed at the center of the cube and all the particles in the model have the same radii. The BCC unit cell is known to be the most isotropic configuration of all unit cell structures. Another advantage of BCC over simple cubic structure is that it takes into effect, to a limited extent, the effect of neighboring particles on the stress and strain distribution around the central inclusion.

In Figure 3.1, the edge length of the cube is fixed at $30 \mu \mathrm{m}$ and the radii of the inclusions are altered to achieve different volume fractions. Though the BCC UCM tries to accommodate multiple particles, it not only idealizes the shape of the particles, but also fixes the inter-particle distance, which is a serious drawback of the model. Two BCC models having $15 \%$ and $20 \%$ reinforcement fraction were produced and the radii of the particles in these models are given in Table 3-1 below.

Table 3.1 - Radii of inclusions for different volume fractions of BCC-UCM

\begin{tabular}{cl}
\hline Volume Fraction & Radius \\
\hline $20 \%$ & $8.63 \mu \mathrm{m}$ \\
$15 \%$ & $7.84 \mu \mathrm{m}$ \\
\hline
\end{tabular}




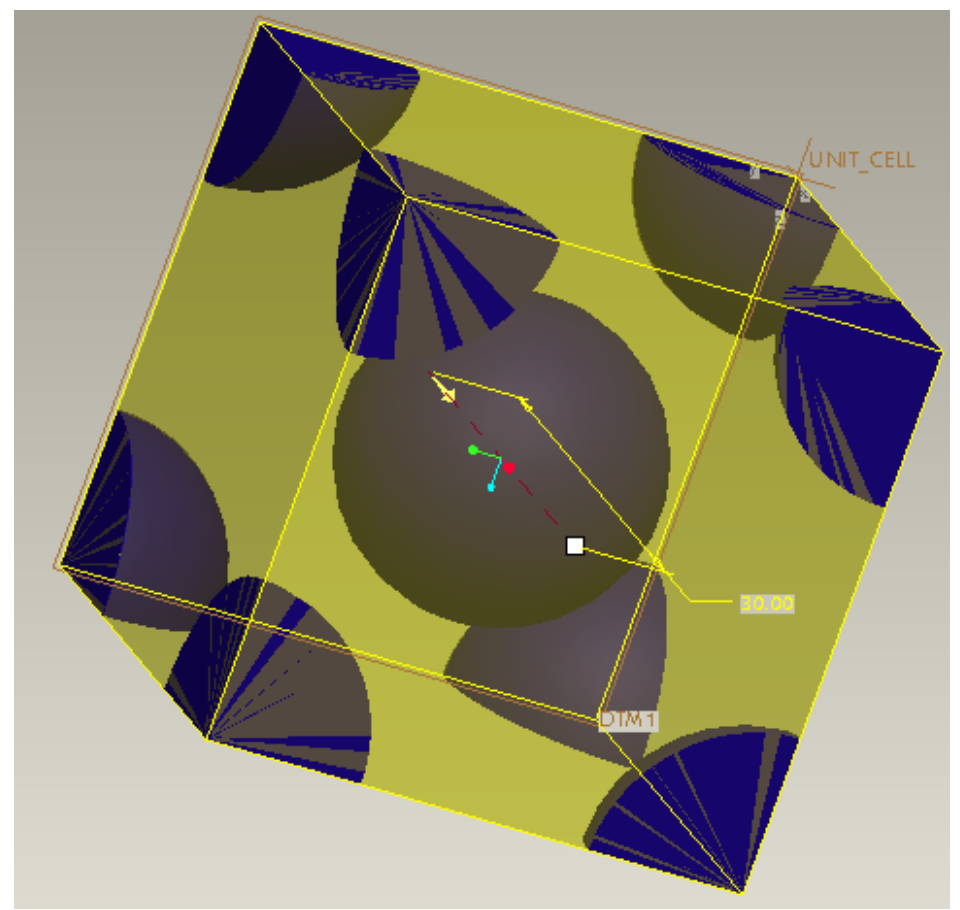

Figure 3.1 - Body centered cubic UCM having a volume fraction of $\mathbf{1 5 \%}$

The model shown in Figure 3.1 is meshed using TrueGrid ${ }^{[46]}$ and the mesh exported to ANSYS ${ }^{[47]}$ for analysis. While meshing, the cube is divided roughly into 27 equal regions, 9 of which are occupied by the particles and the rest by the matrix. An example mesh for a cube having 15\% volume fraction is depicted in Figure 3.2. Due to this methodology of meshing, achieving a volume fraction more than $22 \%$ is not possible. Because of the geometry of the configuration, at high volume fractions, the inner region containing the central inclusion would grow exceedingly big and consequently, the outer regions would be unable to accommodate the particles. Due to this limitation, the unit cell models which were analyzed have a maximum volume fraction of $20 \%$.

The UCM is meshed with eight-noded hexagonal solid elements. The mesh density in the model is higher in the region of the particles which is required to capture the rapidly changing stress-strain field in this region. One of the advantages of using a unit cell model is that it is computationally inexpensive. The above $3 \mathrm{D}$ model is meshed with 13,000 elements, compared to the 65,000 elements used for the meshing of the random inclusion model having the same volume fraction. 


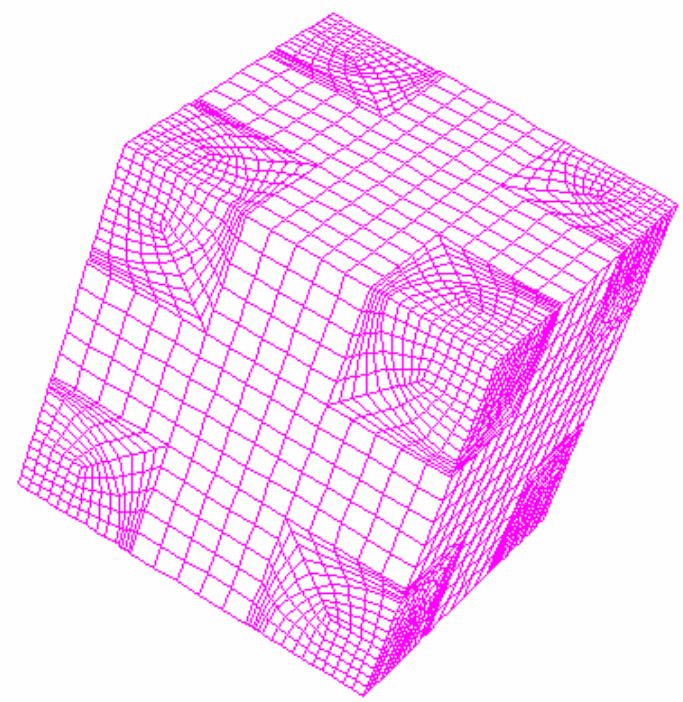

Figure 3.2 - A FE mesh for a UCM with 15\% volume fraction

The model generated in this manner is defined with boundary and loading conditions as explained later, prior to analysis.

\subsection{RVE with randomly distributed spheres}

To solve the issue of fixed inter-particle sizes and inter-particle distances, which is prevalent in the BCC-UCM, an RVE is considered in which the particles are distributed in the matrix in a random manner. Over the years, several strategies have been used to produce these random distribution models (RDMs). Monte-Carlo method ${ }^{[48]}$ and simulated annealing procedures ${ }^{[49]}$ are commonly used, which start with an initial particle arrangement that is continually modified until all the microstructure metrics are fulfilled. In the current study a random sequential adsorption scheme ${ }^{[50]}$ is used, which works by adding one particle after another into the matrix until the required volume fraction is reached. Such a sequential scheme is easy to implement using a prototyping language such as Matlab ${ }^{[51]}$ unlike the other two mentioned methods.

The inclusions were not allowed to impinge on each other and they are not allowed to touch the walls of the cubical RVE either. The radii of inclusions are not constant and varied over a range provided by the user. To estimate the average size of the 
particles and other parameters such as distribution of particles sizes in the material, a scanning electron microscope image of the material, shown in Figure 3.3 was analyzed.

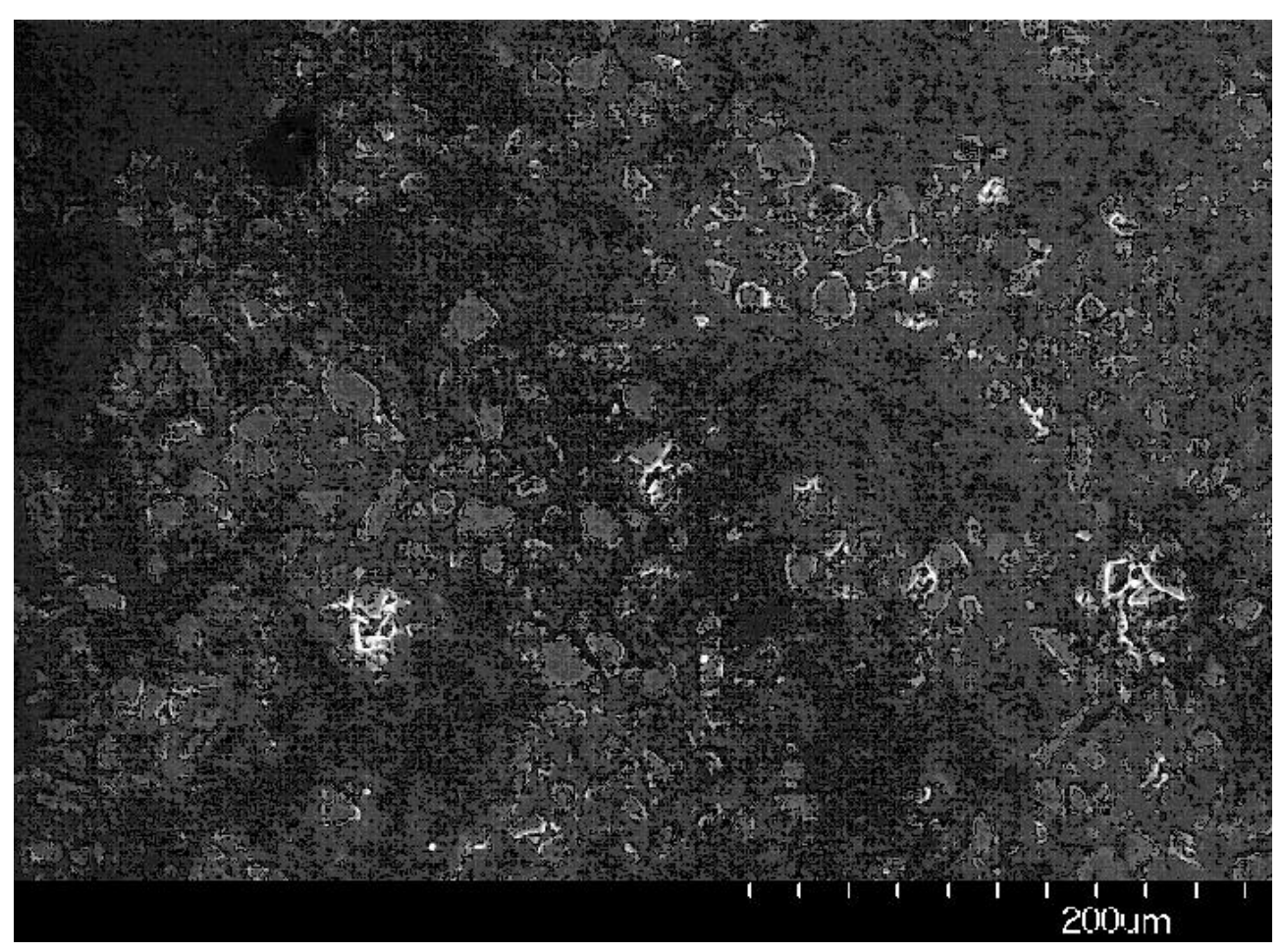

Figure 3.3 - An SEM image of the microstructure of the Al/SiC MMC being studied.

Unlike the BCC-UCM, in which for a given cell size the radius of the particles is dictated by the volume fraction, an RDM of a particular volume fraction can be made of any particle size. The image in Figure 3.3 was digitized and fed to a MATLAB program which estimates the amount of $\mathrm{SiC}$ in the microstructure and by counting the number of particles in a unit area; the average particle size can be estimated.

Another issue to be addressed was the detection of collision of one particle on another and conforming the model to the minimum inter-particle distance. For intersecting inclusions, the difficulty in calculating the intersection volumes between two and multi-particle intersections and consequently, estimating the overall volume fraction would be extremely resource intensive. Thus intersecting inclusions are disallowed in the current model. For the case of spherical inclusions, detecting intersections is relatively simple. If $\left(\mathrm{x}_{0}, \mathrm{y}_{0}, \mathrm{z}_{0}\right)$ and $\left(\mathrm{x}_{1}, \mathrm{y}_{1}, \mathrm{z}_{1}\right)$ are the centers of the two spheres and $\mathrm{r}_{0}$ and $\mathrm{r}_{1}$ are their radii, the spheres are not intersecting if: 


$$
\sqrt{\left(x_{1}-x_{0}\right)^{2}+\left(y_{1}-y_{0}\right)^{2}+\left(z_{1}-z_{0}\right)^{2}} \geq r_{0}+r_{1}
$$

To ensure a minimum inter-particle distance, the minimum value as input by the user is included in the right hand side of the above inequality. It is advisable to include a minimum inter-particle distance in the routine to obtain an efficient mesh of the RVE. If the distance between two adjacent spheres is very low, the meshing program refines the mesh to a very high degree in that region, resulting in a very high node and element count.

To generate the artificial microstructure, a routine was written in MATLAB, whose algorithm is summarized in the flowchart below. The routine take the size of the cube, average radius of the inclusion, range of inclusion radii, volume fraction to be achieved and minimum inter-particle distance as inputs. The output is in the form of an ASCII file which contains the ANSYS commands to generate the desired RVE.

The number of inclusions required to fulfill a given volume fraction is dependent on the mean radius of the inclusion. Thus, smaller the specified inclusion radius, more difficult is it to obtain the required microstructure. Random number generators are used to locate potential positions for the inclusions. A sphere generated at a candidate point is retained only if it does not intersect with any existing spheres or the walls of the cube. If it does, the point is discarded and another point chosen. This is repeated until the combined volumes of the spheres satisfy the specified volume fraction condition. Clearly, this is an iterative process which needs to run for extended periods when microstructures of high volume fraction need to be generated, as indicated by the algorithm shown in Figure 3.4 . 


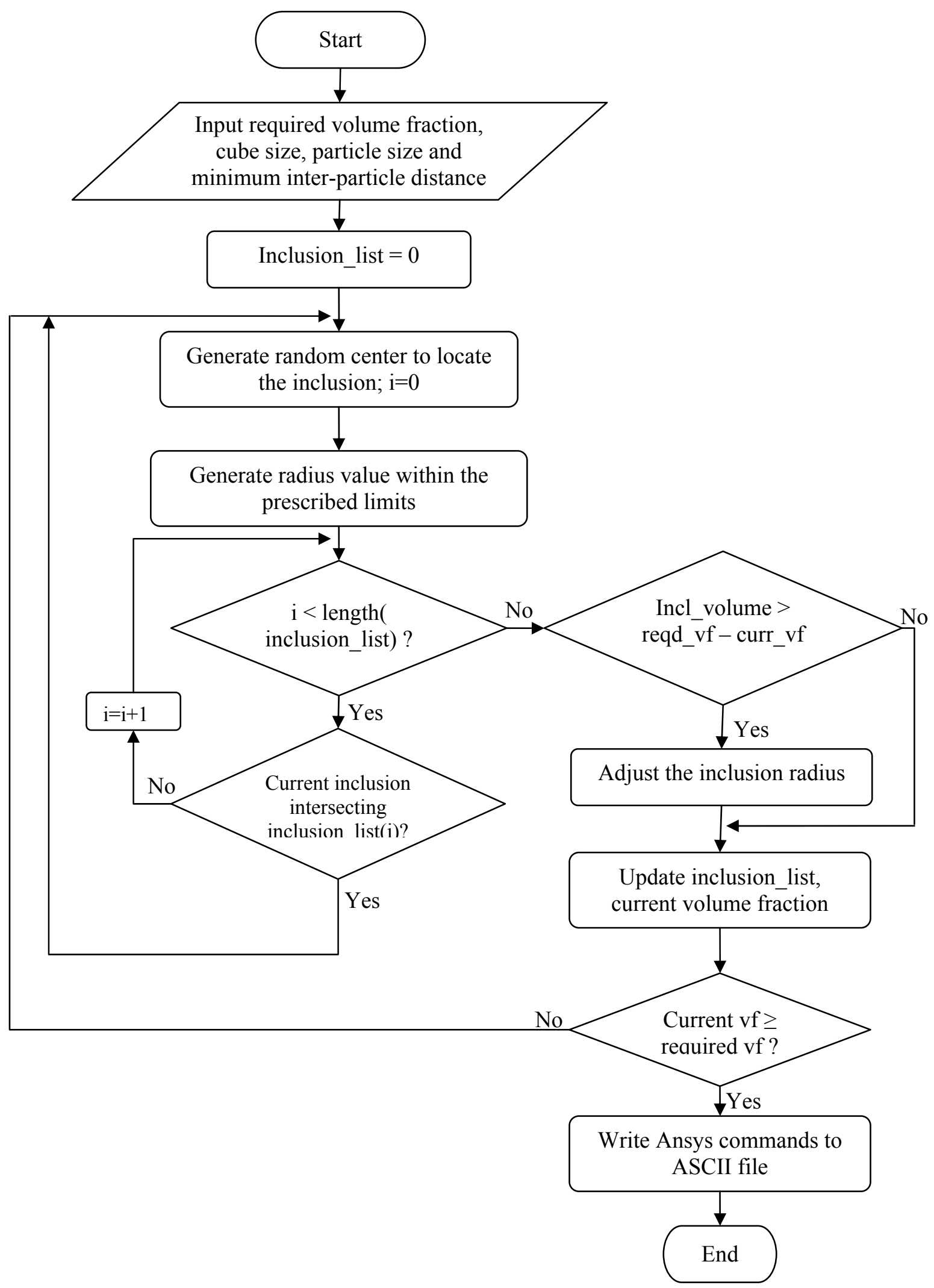

Figure 3.4 - The algorithm for generation of RDM having spherical inclusions 
A microstructure having a volume fraction of $20 \%$, produced from the algorithm shown in Figure 3.4, with inclusions of average radius $9 \mu \mathrm{m}$ is shown in Figure 3.5. It can be seen that the radii of the inclusions are not constant and that no sphere intersects with any other sphere or the side walls of the cube.

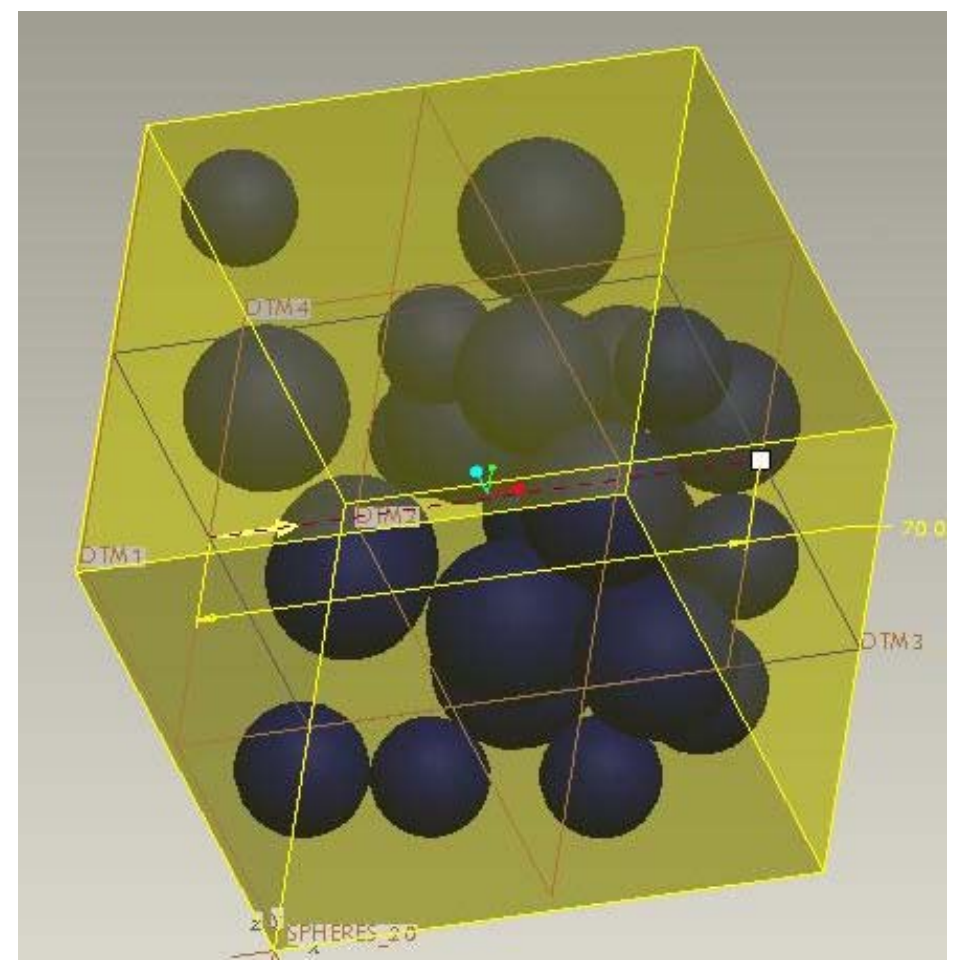

Figure 3.5 - RDM of $20 \%$ volume fraction having spheres of average radius $9 \mu \mathrm{m}$ as inclusions

The ASCII file generated as the output of the Matlab routine contains the commands required to recreate the microstructure in ANSYS. The ASCII file can be read directly by ANSYS as an input macro. Boolean overlap operation ${ }^{[47]}$ which is essential to separate the inclusion surrounding matrix volumes into separate entities, is performed on the microstructure before meshing it with 10 noded tetrahedrons. An example Matlab routine along with the generated ANSYS input macro is given in Appendix C.

Due to the random nature of the geometry, obtaining a mapped mesh is extremely difficult, which rules out hexahedral elements in ANSYS. Meshing the entire geometry with 4 noded tetrahedral elements might result in a higher overall stiffness value and so, a higher order tetrahedral element was chosen. The number of nodes and elements in the mesh varied based on the volume fraction of the microstructure, with a higher number of 
elements generated for higher volume fractions. This was due to smaller elements produced inside the inclusions than in the matrix. Two stages of progressively finer meshes were used on the models to check variations in the solutions. For a volume fraction of $20 \%$, an average of 65,000 elements were used to mesh the microstructure. Due to the limitation on the number of elements and nodes that could be used in ANSYS, further mesh refinement could not be carried out. The RDM shown in Figure 3.5 is shown after meshing in Figure 3.6. After meshing, the model is defined with the required boundary conditions, loading conditions and processed.

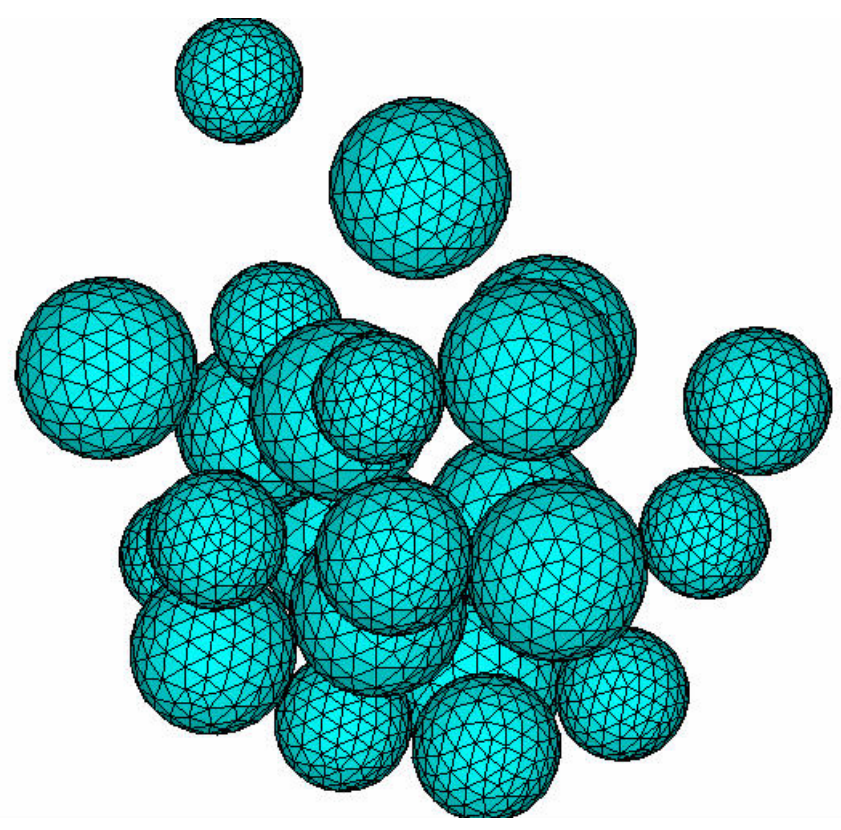

Figure 3.6 - Meshed spehrical inclusions of a $20 \%$ volume fraction microstructure.

\subsection{RVE with randomly distributed ellipsoids}

Though RVEs having randomly embedded spheres are a better representation than traditional UCMs, they still suffer from the drawback of over-simplifying the shape of the inclusion particles. Representing the true shapes of inclusions is a very difficult task because of their completely arbitrary shape and size. It has been noted in previous studies [14-17] that the closest approximation to arbitrarily shaped particles is in the form of ellipsoids and so it makes it worthwhile to have a model with randomly embedded ellipsoids instead of spheres. But using ellipsoids instead of spheres increases the 
complexity of the microstructure generation by several orders. Unlike spheres, ellipsoids not only have three radii, but also three orientations with respect to three coordinate axes. To obtain an isotropic microstructure, the orientations of the ellipsoids had to be completely random. In the present study, the ellipsoids generated are limited to prolate spheroids. If $\mathrm{a}, \mathrm{b}$, and $\mathrm{c}$ are the diameters in the $\mathrm{x}, \mathrm{y}$ and $\mathrm{z}$ directions respectively, as shown in Figure 3.7, the class of ellipsoids in which $b=c<a$ are defined as prolate spheroids. They are solids obtained from revolving ellipses about the major axis. Thus, prolate ellipsoids need only two radii to define them, unlike scalene ellipsoids which require three.

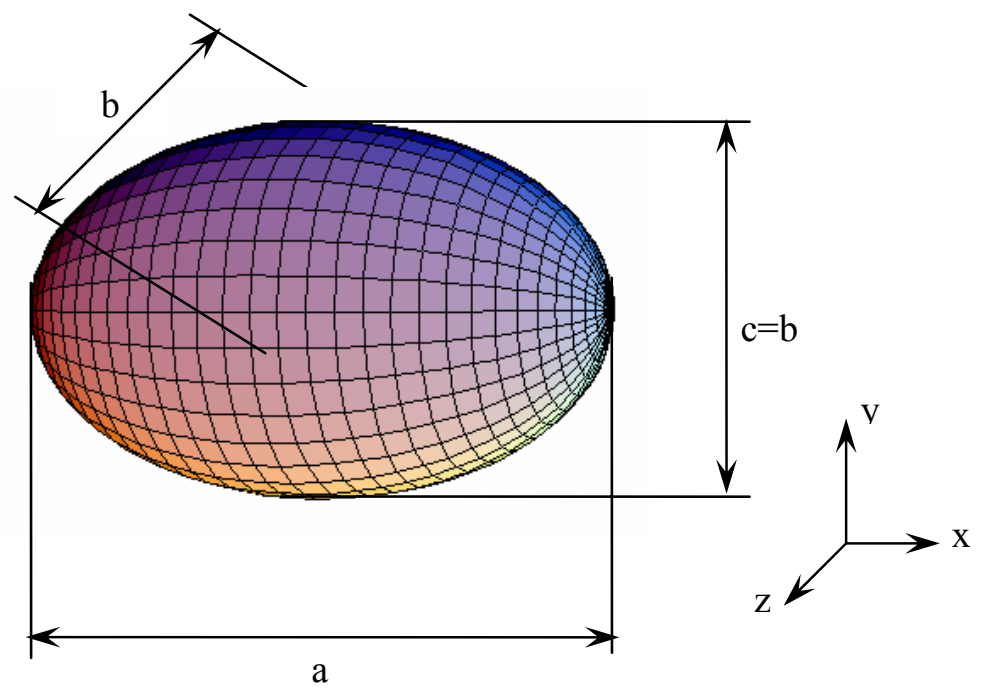

Figure 3.7 - A prolate ellipsoid which has the two minor radii equal to each other

To obtain ellipsoids of different aspect ratios, the radius specified by the user was multiplied by a factor greater than one to obtain the major radius and a factor lesser than one to obtain the minor radius. That is,

$$
\begin{array}{ll}
\text { major radius } & a=\text { specified radius } \times(1+x) \\
\text { min or radius } & b=\text { specified radius } \times(1-x)
\end{array}
$$$$
\text { where } x=\text { random number between } 0 \text { and } f \text { (specified by the user) }
$$

In the current study, the factor $\mathrm{f}$ has a fixed value of 0.25 and $\mathrm{x}$ can assume a value of zero. i.e a few spheres may also be generated among the ellipsoids. 
One of the issues with implement models having ellipsoids as inclusions is the detection of intersection between ellipsoid shaped inclusions. While a simple closed-form solution can be worked out for spheres, no such solution exists for quadric surfaces. Recent methods to determine the distance between two ellipsoids involve use of theorems in line geometry as demonstrated by Sohn et al ${ }^{[32]}$. However, for this method to be efficient, a parametric representation of the ellipsoids is required, which is not easy to obtain. Choi et al ${ }^{[33]}$ used an algebraic condition to determine if ellipsoids moving in a predetermined path collide with each other at any instant of time. This algebraic principle was best summarized by Wang et al ${ }^{[34]}$ and in slightly different terms by Alfano and Greer $^{[35]}$.

An ellipsoid which has its center at the origin and diameters $a, b$ and $c$ is represented in matrix form using generalized coordinates as:

$$
X^{T} A X=0 \quad \text { where } A=\left(\begin{array}{cccc}
1 / a^{2} & 0 & 0 & 0 \\
0 & 1 / b^{2} & 0 & 0 \\
0 & 0 & 1 / c^{2} & 0 \\
0 & 0 & 0 & -1
\end{array}\right) \text { and } X=\left\{\begin{array}{l}
x \\
y \\
z \\
1
\end{array}\right\}
$$

A translation is applied to this ellipsoid to move the center from the origin to any point $\left(\mathrm{x}_{0}, \mathrm{y}_{0}, \mathrm{Z}_{0}\right)$. The translation is represented as:

$$
X^{T} T^{T} A T X=0 \quad \text { where } T=\left(\begin{array}{cccc}
1 & 0 & 0 & 0 \\
0 & 1 & 0 & 0 \\
0 & 0 & 1 & 0 \\
-x_{0} & -y_{0} & -z_{0} & 1
\end{array}\right)
$$

For the problem of ellipsoidal intersection detection, consider two ellipsoids,

$$
\begin{aligned}
& X^{T} A X=0 \\
& X^{T} B X=0
\end{aligned}
$$

the characteristic equation of these two ellipsoids is defined as: 


$$
\operatorname{det}(A-\lambda B)=0
$$

It is known that any affine transformation or coordinate rotation applied to the matrices $\mathrm{A}$ and $\mathrm{B}$, while changing the characteristic equation itself, does not change the roots of the characteristic equation. These roots of the characteristic equation are made use of to detect collision between the ellipsoids. It is shown in [33] that there always exists a set of at least two distinct negative roots for the above characteristic equation when the ellipsoids are not intersecting each other. It is to be noted that the task of finding the roots of the characteristic equation is equivalent to determining the generalized eigenvalues for $\mathrm{A}$ and $\mathrm{B}$. Thus, if there at least two distinct negative generalized eigenvalues for matrices $\mathrm{A}$ and $\mathrm{B}$, the two ellipsoids are not touching each other.

The above algebraic principle is used in the algorithm to generate microstructures having ellipsoidal inclusions. The ellipsoidal algorithm is similar to the spherical algorithm but for a few additional steps. Along with the affine transformation explained above, additional coordinate rotation transformations at random angles are applied to the ellipsoids. Nested coordinate rotations, first about the x-axis and then the $y$-axis are applied to obtain randomly oriented spheroids. Intersection of spheroids is checked only after application of rotation and affine transformations. The other change is in the calculation of volume of ellipsoids, which is given by:

$$
V=\frac{4}{3} \pi a b c
$$

A flowchart summarizing the algorithm is shown Figure 3.8 .

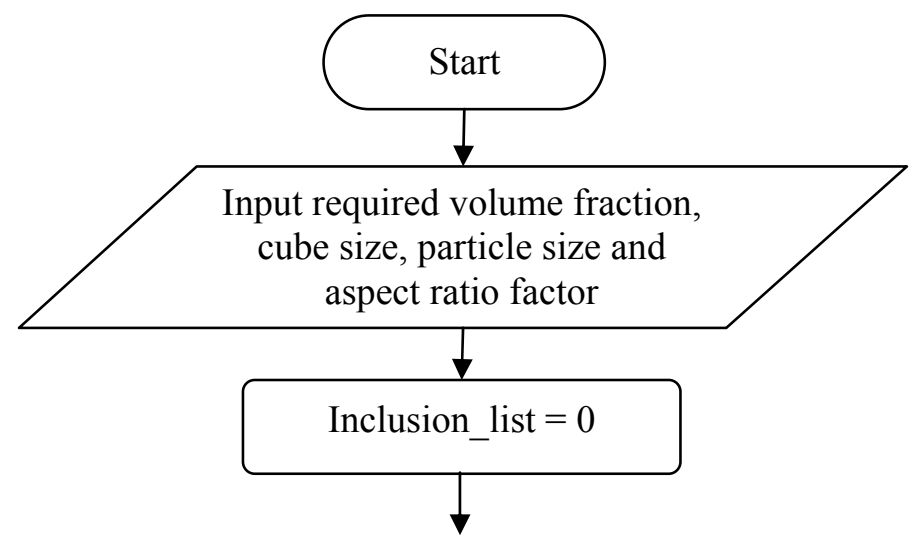




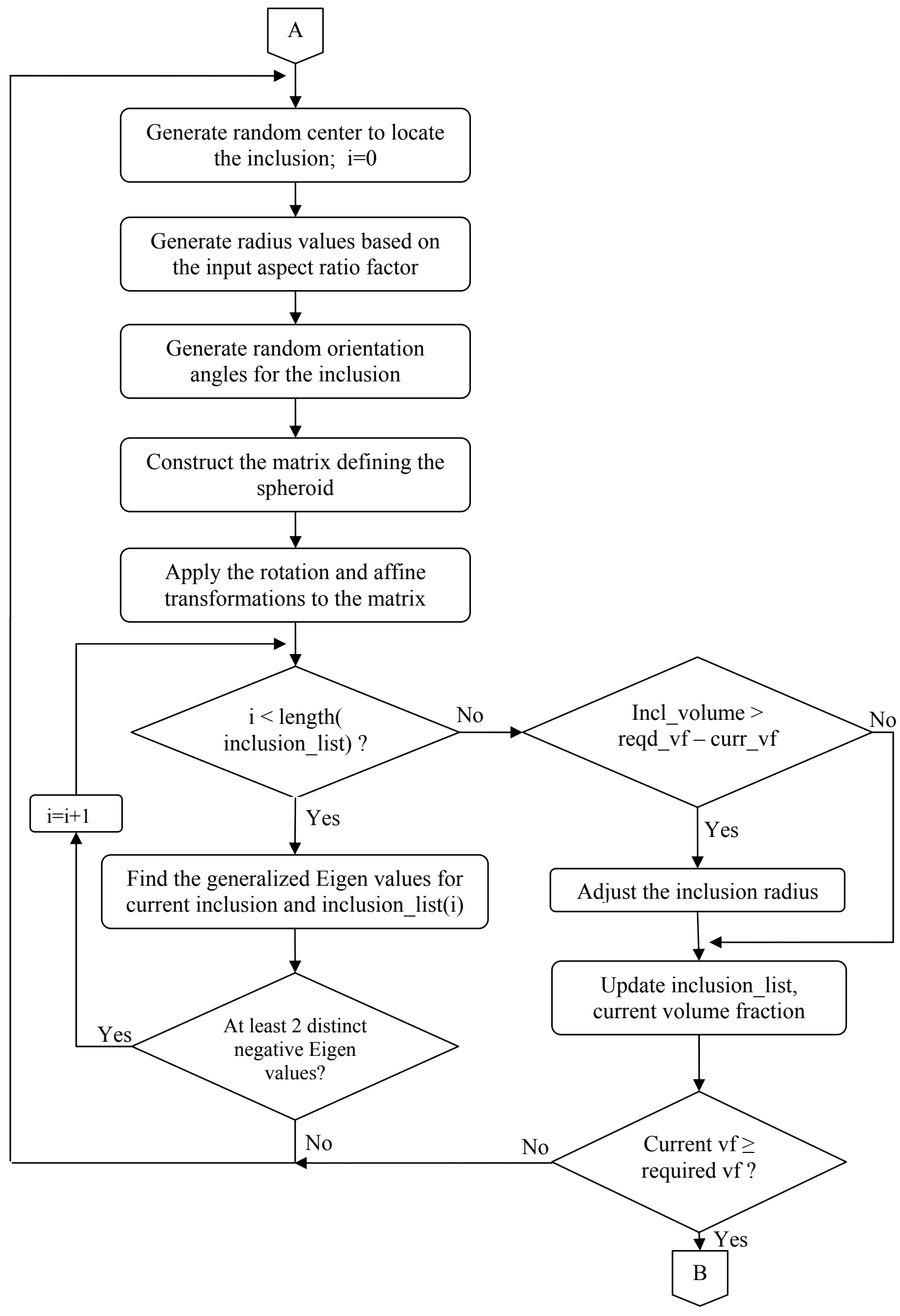




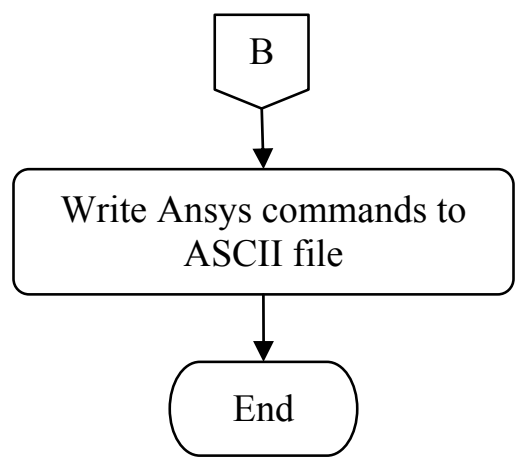

Figure 3.8 - The above flowchart summarizes the subroutine used to generate microstructure containing ellipsoid shaped inclusions.

For the determination of Eigen values of the characteristic matrix, the QZ algorithm ${ }^{[51]}$ was used; ignoring the symmetry of matrices A and B. This was necessary as the range of coefficients in the matrices were very large. An example microstructure generated by the above algorithm is shown in Figure 3.9.

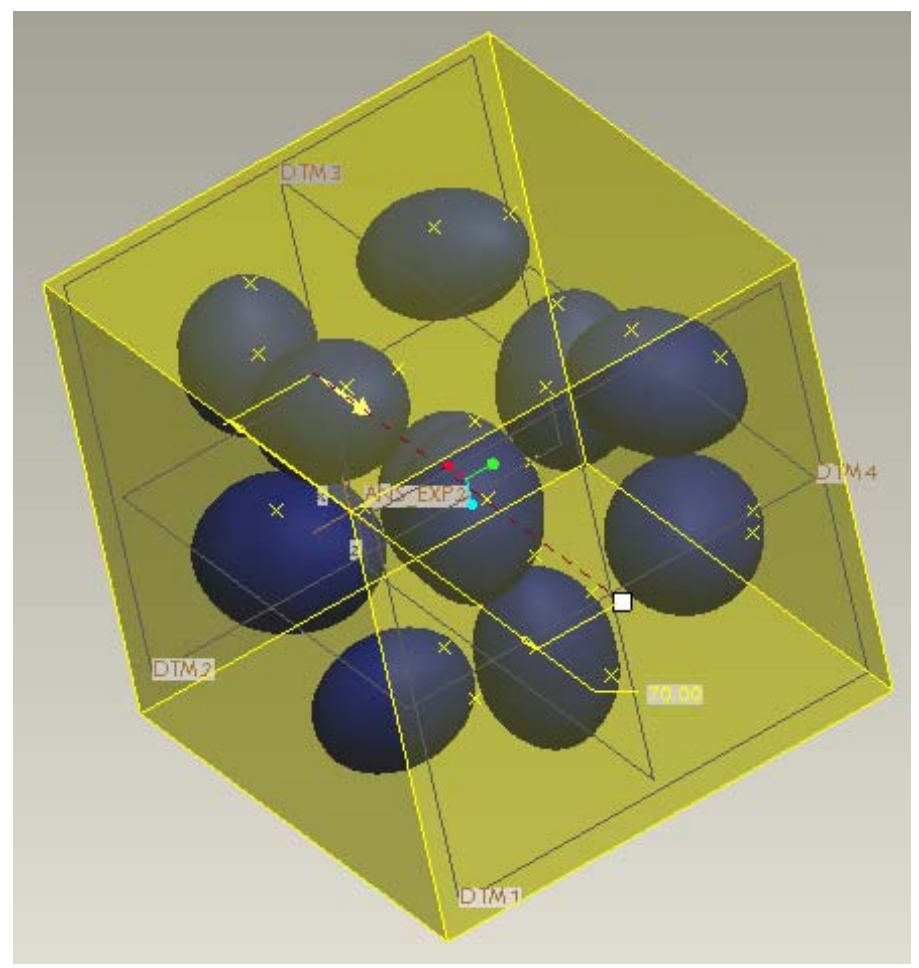

Figure 3.9 - An example microstructure containing ellipsoidal inclusions and having volume fraction of $15 \%$. 
Similar to the spherical RDM, the output of the subroutine is an ASCII file containing the commands to the regenerate the microstructure in ANSYS. After performing Boolean operations on the microstructure, it is meshed with 10-node tetrahedral elements. The inclusions and matrix is defined with appropriate material models and boundary, loading conditions are defined prior to solving.

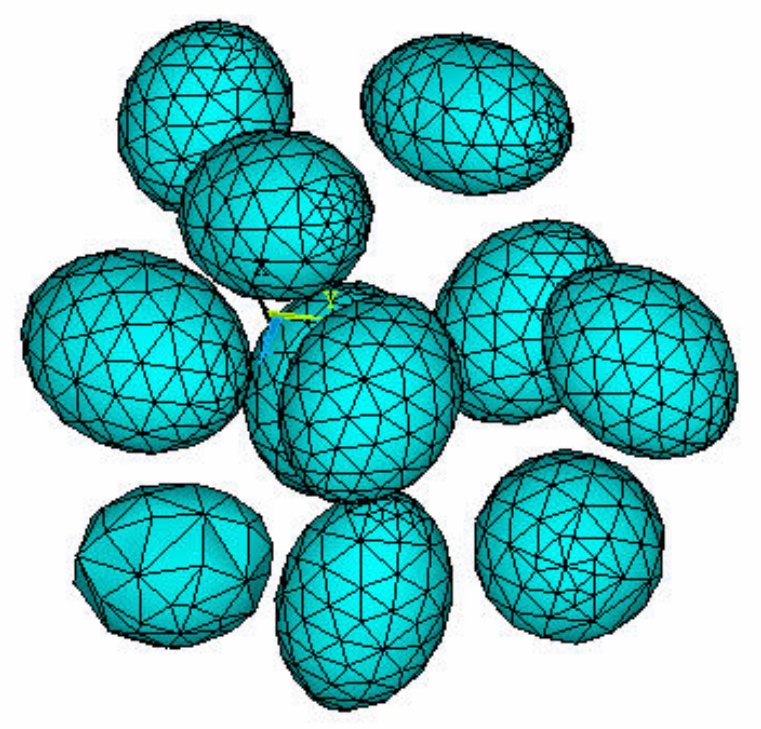

Figure 3.10 - Meshed ellipsoidal inclusions of a 15\% volume fraction microstructure. Note that the matrix surrounding the inclusions is not shown in the figure.

For microstructures meshed in this manner, an average of $65,000-70,000$ elements were required.

\subsection{Material Models}

To obtain the elastic and plastic response of the overall MMC, it is important to incorporate realistic material models in the FE representation. In the current study for all three types of models explained above, the aluminum matrix is assumed to be an elasticplastic material and the inclusions are approximated as being made of linear-elastic silicon carbide. The interface between the matrix and the inclusions is assumed to be ideal and particle cracking is not considered. The properties of aluminum and $\mathrm{SiC}$ as defined in the FE models are listed in Table 3-2. 
Table 3.2 - Properties of matrix and inclusion materials used in the FE model

\begin{tabular}{ccc}
\hline Material & Young's Modulus (GPa) & Poisson's Ratio \\
\hline Silicon Carbide & 410 & 0.14 \\
Aluminum Al 6061-T6 & 72 & 0.33 \\
\hline
\end{tabular}

Further, the elastic nature of aluminum is approximated by using Multilinear kinematic hardening rule using von-Mises plasticity. This is achieved by providing various points on the stress-strain curve of aluminum as input to the material model, along with the Young's modulus and Poisson's ratio.

\subsection{Boundary conditions and loading}

One aspect of micromechanical models which affects their performance along with their response is the boundary conditions imposed on them. Traditionally, three faces of the cube are constrained with symmetric boundary conditions and the other three with uniform displacement conditions. The uniform displacement condition is often imposed so as to make the calculation of overall strains easy, but this condition leads to unrealistic stresses being induced inside the model.

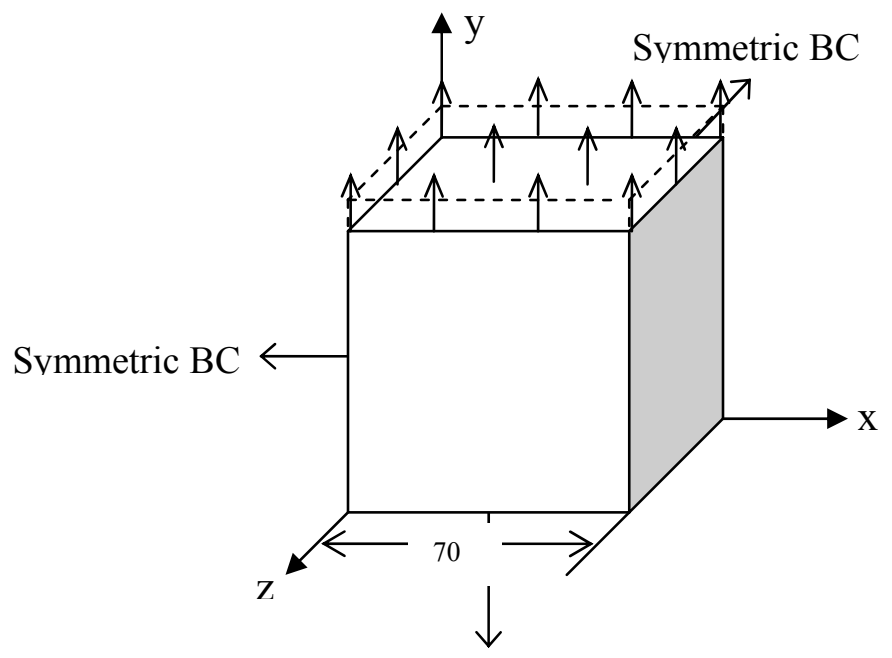

Symmetric BC

Figure 3.11 - Schematic of loading and boundary conditions imposed on the models 
Though a model having no boundary conditions and loading on two opposite faces would be a better representation of the condition in the material during uniaxial test, such a model has difficulty converging to a solution. Additionally, it will not represent the confinement condition of the RVE within the whole composite. Thus, in this study, the faces, $\mathrm{x}=0 ; \mathrm{y}=0 ; \mathrm{z}=0$, were constrained with symmetric boundary conditions.

For all the three models explained above, loading was applied in the form of a tensile pressure load to the $\mathrm{y}=\mathrm{a}$ face of the cube, where a is the length of the cube as depicted in Figure 3.11. A pressure of magnitude of $320 \mathrm{MPa}$ was applied incrementally in the form of several load sub-steps. To obtain the overall stress-strain response of the composite, the loading was spread over 10 sub-steps and at each sub-step, the overall stress and strain experienced by the model was calculated using the method described below.

\subsection{Post-Processing}

Post-processing is one of the most resource intensive and time consuming activities in the analysis. After solving, the solution of the analysis is stored in .RST files which have to be queried for obtaining the required information. The primary focus of post-processing is to determine the overall properties of the composite from the FE solution. There are several homogenization techniques available to determine the stress and strain in the overall composite. One of the methods is to calculate the strains from the displacement of the faces of the cube and the stress from the applied load. But a more accurate estimate of the overall stresses and strains can be obtained from considering a weighted volume average of elemental stresses and strains. i.e.,

$$
\begin{gathered}
\bar{\varepsilon}_{i j}=\frac{1}{V} \int_{V} \varepsilon_{i j} d V=\frac{1}{V} \sum_{m=1}^{N} \varepsilon_{i j}^{(m)} V^{(m)} \\
\bar{\sigma}_{i j}=\frac{1}{V} \int_{V} \sigma_{i j} d V=\frac{1}{V} \sum_{m=1}^{N} \sigma_{i j}^{(m)} V^{(m)}
\end{gathered}
$$

where, $\mathrm{V}^{(\mathrm{m})}$ is the volume of element $\mathrm{m}$ 
$\sigma^{(m)}$ is the stress in element $m$

$\varepsilon^{(\mathrm{m})}$ is the strain in element $\mathrm{m}$.

$\mathrm{N}$ is the total number of elements in the model

and $\quad \mathrm{V}$ is the total volume of the cube.

Thus, the stresses and strains at each element has to be used to obtain the overall stress and strain. This volume weighted averaging has to be performed at each sub-step so as to trace an overall stress-strain curve from the points obtained at each sub-step. The ANSYS APDL is made use of in generating a script which performs the necessary postprocessing activities. At the end of post-processing, the required information is written into an ASCII file. The information in these ASCII files is input to the mesoscale model during the second stage of analysis.

\subsection{Model Validation}

Traditionally, mechanical characterization of materials has been carried out solely by conducting various types of static tests on specimen of the material in question. Similarly, in the current study, experimental evaluation was used not only to determine the properties of the material, but also serve as a validation mechanism for the FE models. Since the MMC contains $\mathrm{SiC}$ in particulate form, the over material behaves isotropically, which enables the use of testing standards as specified by ASTM for isotropic materials. The Duralcan brand of $\mathrm{Al} / \mathrm{SiC} \mathrm{MMC}$, manufactured by the Alcan Corporation was used in the study. The material was supplied in the form of cast bricks of aluminum with $20 \% \mathrm{SiC}$ reinforcement by volume.

\subsubsection{Machining the specimen}

It is widely known that metal matrix composites are some of the most difficult materials in terms of machinability. Silicon carbide, which is itself used as a coating for cutting tools, is a highly abrasive material capable of wearing out even the toughest materials. Due to its presence in the MMC, conventional machine tools could not be used to reduce the MMC brick to the required shape. One option was to use specialized 
diamond tipped tools such as diamond tipped mill cutters, grinding wheels, a drilling bit for machining. Due to the large number of tools required for primary and secondary operations and the associated high cost, this option was not explored.

Instead, an Electrical Discharge Machine (EDM) was used for machining the material. The EDM works on the principle that an electric arc formed between the material and electrode tool erodes the material and provides a cutting action. The EDM system consists of the part, which is connected to a power supply and a wire electrode. A potential difference is created between the electrode and the part by using a step-up transformer. The eroded material is removed by a coolant fluid which flows along the cutting wire. Figure 3.12 shows the EDM that was used to machine the specimen in the current study. The cutting pattern of the EDM is CNC controlled, with the shape to be machined input through a modeling program.

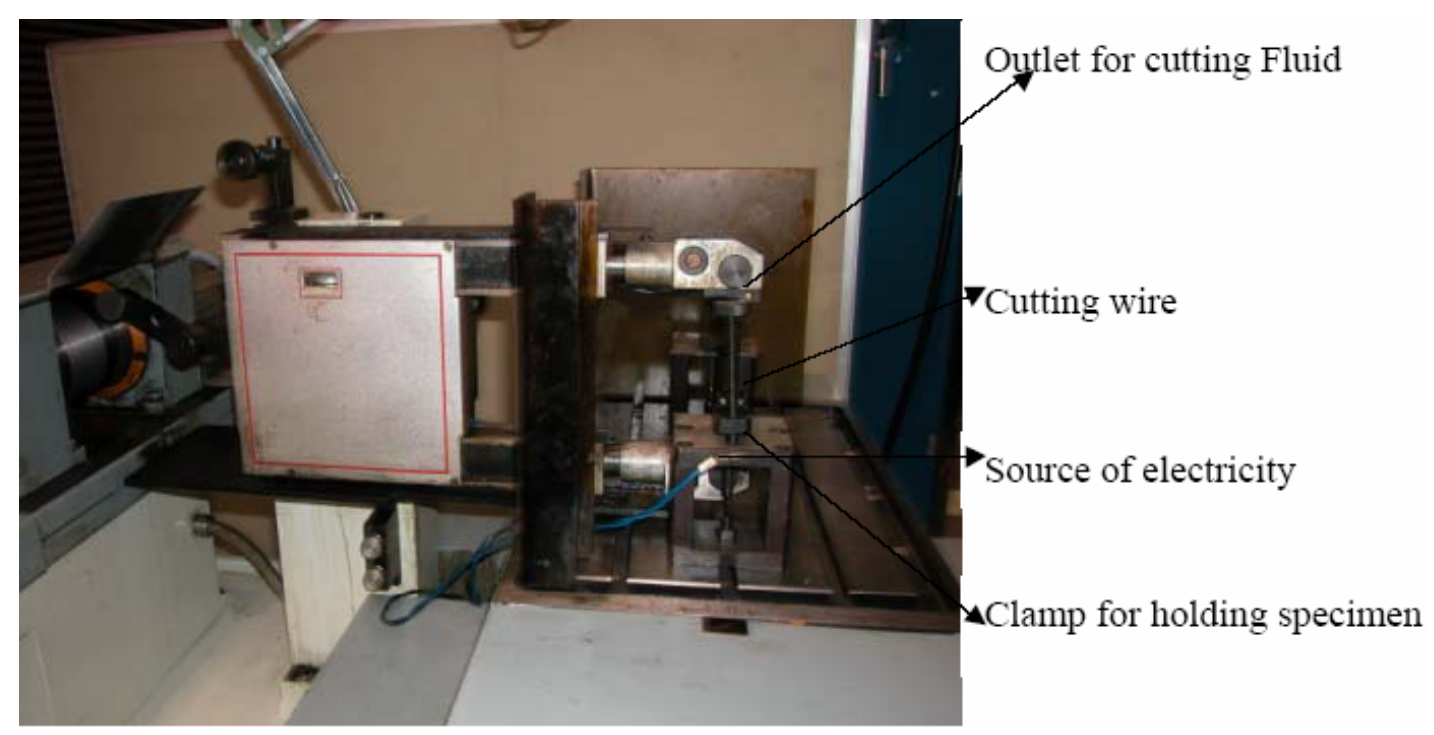

Figure 3.12 - The EDM machine used to reduce the MMC bricks to the required specimen shapes.

The EDM is first used to cut the MMC brick into thin slices of the required thickness and the dog-bone and rectangular patterns were cut into these slices to produce the final specimen shapes. Due to the large size of the brick, the cutting rate was kept low and it took up to 6 hours to machine one completed dog-bone specimen. 


\subsubsection{Experimental Plan}

Since particulate reinforced metal matrix composites are isotropic in nature, the ASTM standard method for tensile testing of metals was chosen (ASTM E8). The dogbone specimen dimensions as specified by the standard are shown in Figure 3.13. Three specimens were tested for the $20 \%$ volume fraction material and two for the $15 \%$ volume fraction material.

The aim of tensile testing was to determine the Young's modulus and the ultimate strength of the material and compare them with the values predicted by the different finite element models. During testing of the tensile specimen, it was seen that the fracture occurred in the reduced section, but close to the gripping area. Since the results from these specimens could not be completely trusted due to the brittleness of MMC, three point bending tests were used as an alternative to determine the bending modulus of the material.
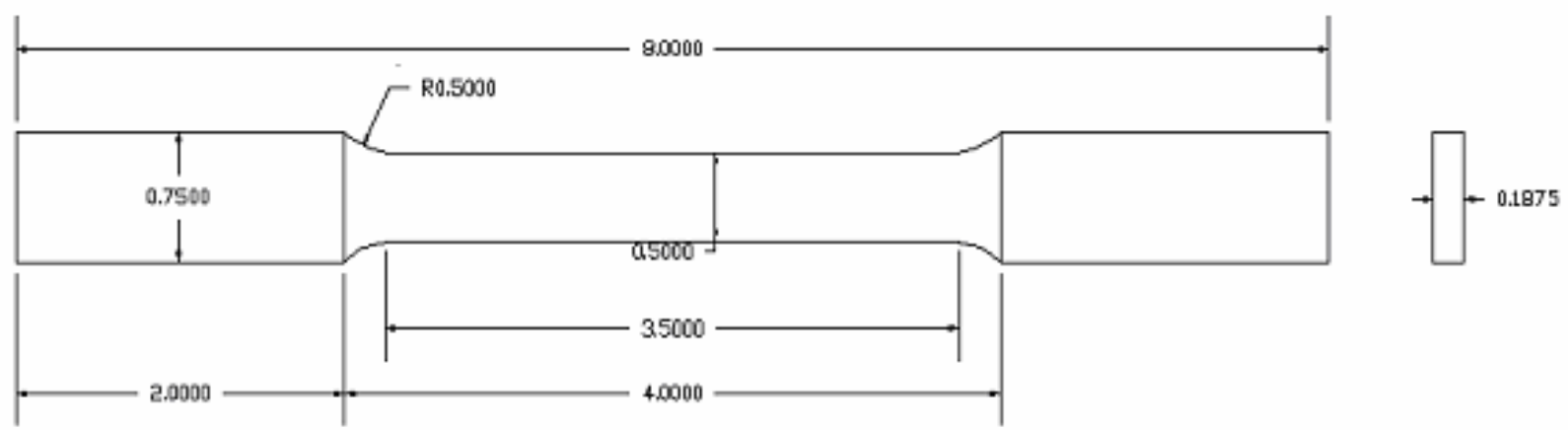

Figure 3.13 - Dimensions of dog-bone specimen as specified by the ASTM E8 standard.

Further, determination of the bending modulus using the 3-point bending test is simpler because it makes use of the load-displacement curve rather than the stress-strain curve, eliminating the use of strain gauges. The 3-point bending tests were carried out according to the ASTM D790 standard specifications. The dimensions of the specimen used are shown in Figure 3.14. 


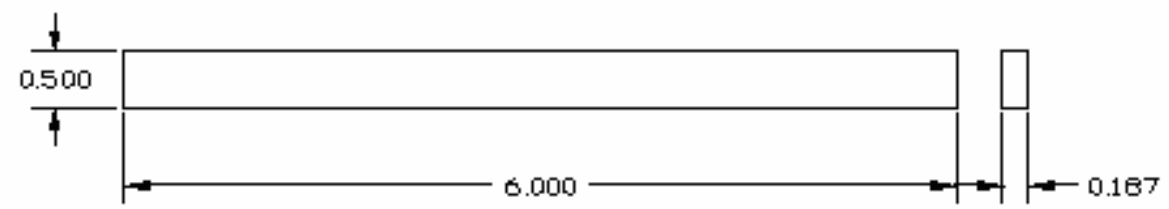

Figure 3.14 - Dimensions of the specimen used for three point bending tests. All dimensions are in inches.

\subsubsection{Test Procedure}

Prior to conducting the tension test, $120 \Omega$ strain gauges were bonded onto the dog-bone shaped specimen to measure longitudinal strains. Leads were soldered on to the strain gauge and connected to the Micro-measurement SYSTEM 5000 data-acquisition system. The loading was done through an MTS testing machine, with a programmable controller. The MTS machine is also connected to the data-acquisition system so as to record the load and the displacement of the crosshead.

The specimen was gripped using wedge-grips and special care was taken to see that the specimens were aligned with the grips, so as to eliminate bending and twisting effects while loading. The loading of the specimen was displacement controlled and it was at a rate of $0.005 \mathrm{in} / \mathrm{min}$. Strain, displacement and applied load were measured every $1 / 10^{\text {th }}$ of a second and recorded by the data-acquisition system. STRAIN SMART software package ${ }^{[53]}$ was used to record the data acquired from the system. The recorded data was exported as an ASCII file, from which further data analysis was carried out.

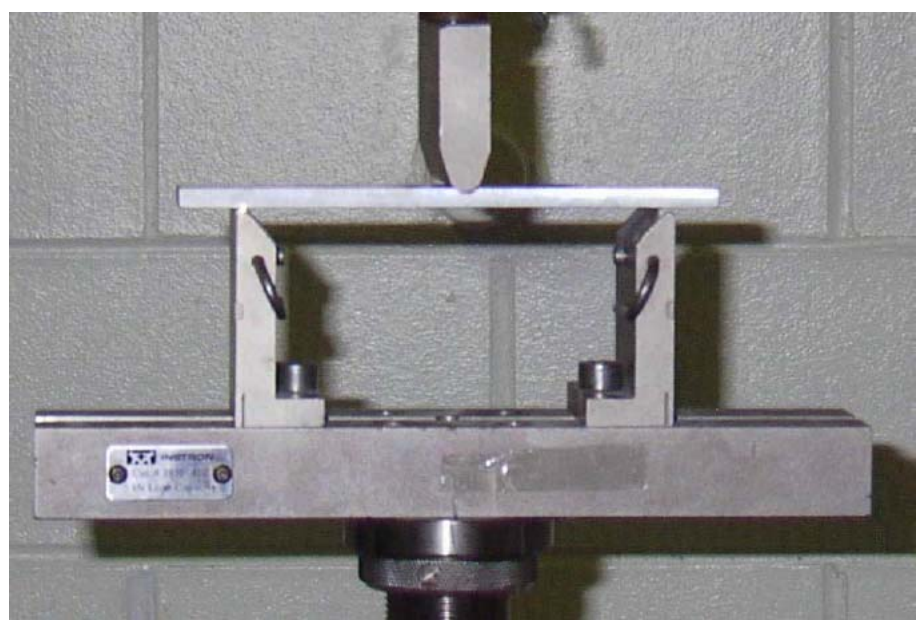

Figure 3.15 - Three point bending testing of MMC specimen using an INSTRON machine. 
For the three-point bending tests, the INSTRON machine, as shown in Figure 3.15, was used due to the reduced load required to attain failure. Since only the load and transverse displacement were required, no external data acquisition system was used. The same loading rate as the tension test was maintained through a computer controlled loading arm. A load cell connected to the arm was used to record the applied load.

The load and displacement recorded in this manner was exported to an ASCII file, which was plotted against each other and the slope of the linear part of the curve was used in the estimation of the Young's modulus using the ASTM standard relation,

$$
\begin{aligned}
& \qquad E_{b}=\frac{L^{3} m}{4 b d^{3}} \quad \text { where } \\
& L=\text { Support span } \\
& E_{b}=\text { Modulus of elasticity } \\
& b=\text { width of the beam } \\
& d=\text { depth of beam } \\
& m=\text { slope of tan gent to the initial part of load - displacement curve }
\end{aligned}
$$

One important aspect about the material being tested, which should be noted is that the specimen generated from the EDM were not subjected to any heat-treatment. So, the amount of residual stresses present in the material after cutting is not high. This has a large effect on the strength values as recorded by tensile tests. The results of the experimental evaluation along with a comparison with the values predicted by the FE models are presented in the next chapter. 


\section{Chapter Four}

\section{Results and Discussion}

The distribution of stresses and strains across the microstructure is discussed under the microscopic response subheading, while a comparison of overall material properties as predicted by the two RDMs, UCM and random array model are summarized in the macroscopic response model. Though models having various volume fractions were analyzed in this study, most of the discussion is about models having $15 \%$ or $20 \%$ volume fractions as these are the materials with maximum application.

\subsection{Microscopic Response}

Analyzing the distribution of stresses and strain in the microstructure of the MMC gives an insight into the overall behavior of the composite and enables designing better performing materials. However each FE model developed predicts a different microscopic behavior. In most of the cases, we consider the distribution of von-Mises equivalent stress or distribution of maximum principal stress as they provide a good indication of points of failure initiation.

\subsubsection{Unit Cell Model}

The distribution of stresses and strains in the unit cell models is mostly symmetric due to the inherent symmetry present in the model and the kind of boundary conditions imposed. It is well-known that boundary conditions greatly affect the results of the UCMs and that even a slight change like imposing uniform displacement condition across any face greatly changes the overall response of the composite.

The distribution of von Mises equivalent stress in a section of $20 \%$ volume fraction UCM, which is loaded just beyond yielding, is shown in Figure 4.1. Maximum principal stress in the material is shown in Figure 4.2. Vertical bands of equal stress are formed due to the symmetry of the model. This type of distribution is not seen in the other two types of models analyzed. 


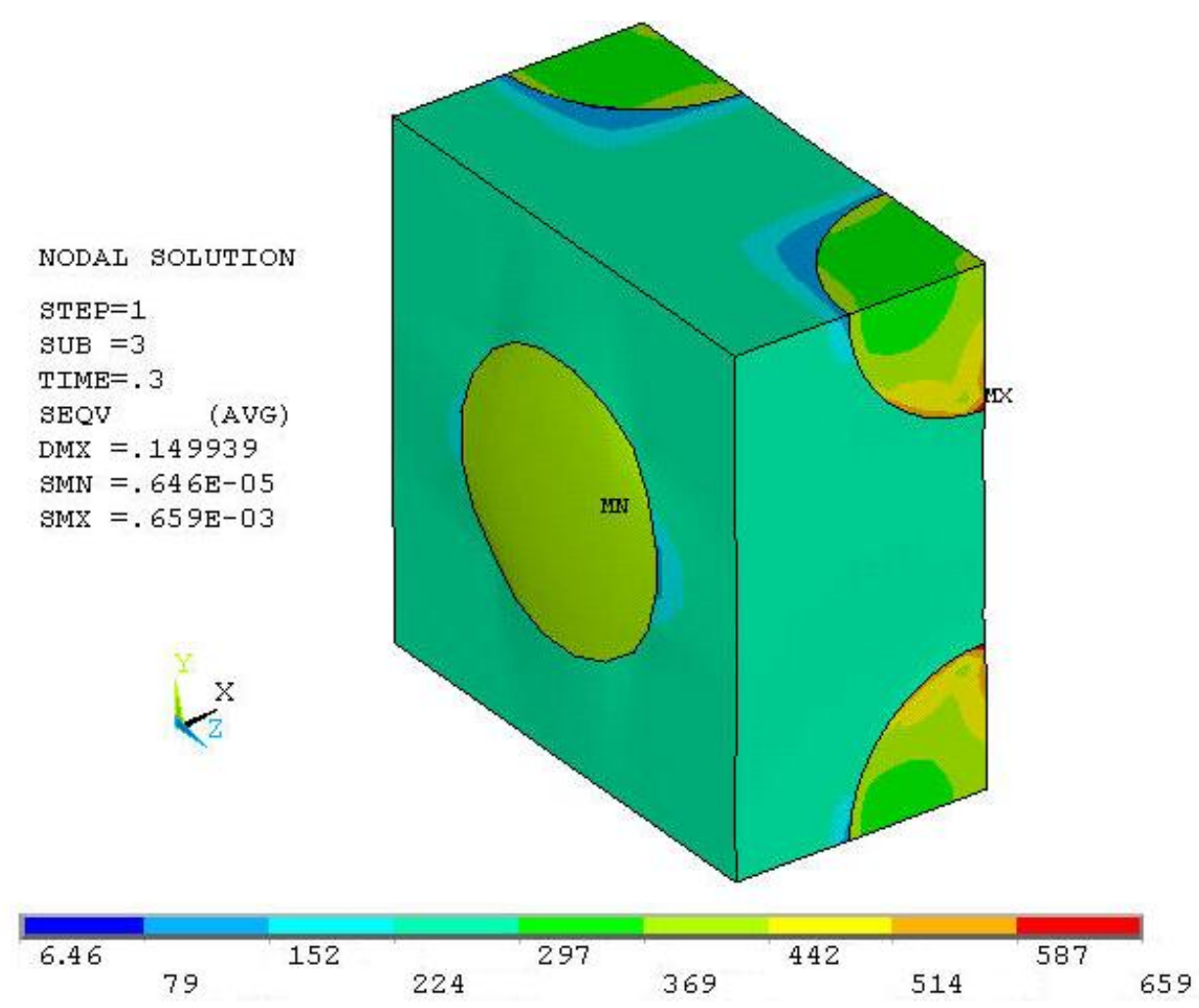

Figure 4.1- Distribution of von-Mises stress (MPa) in a $20 \%$ volume fraction UCM

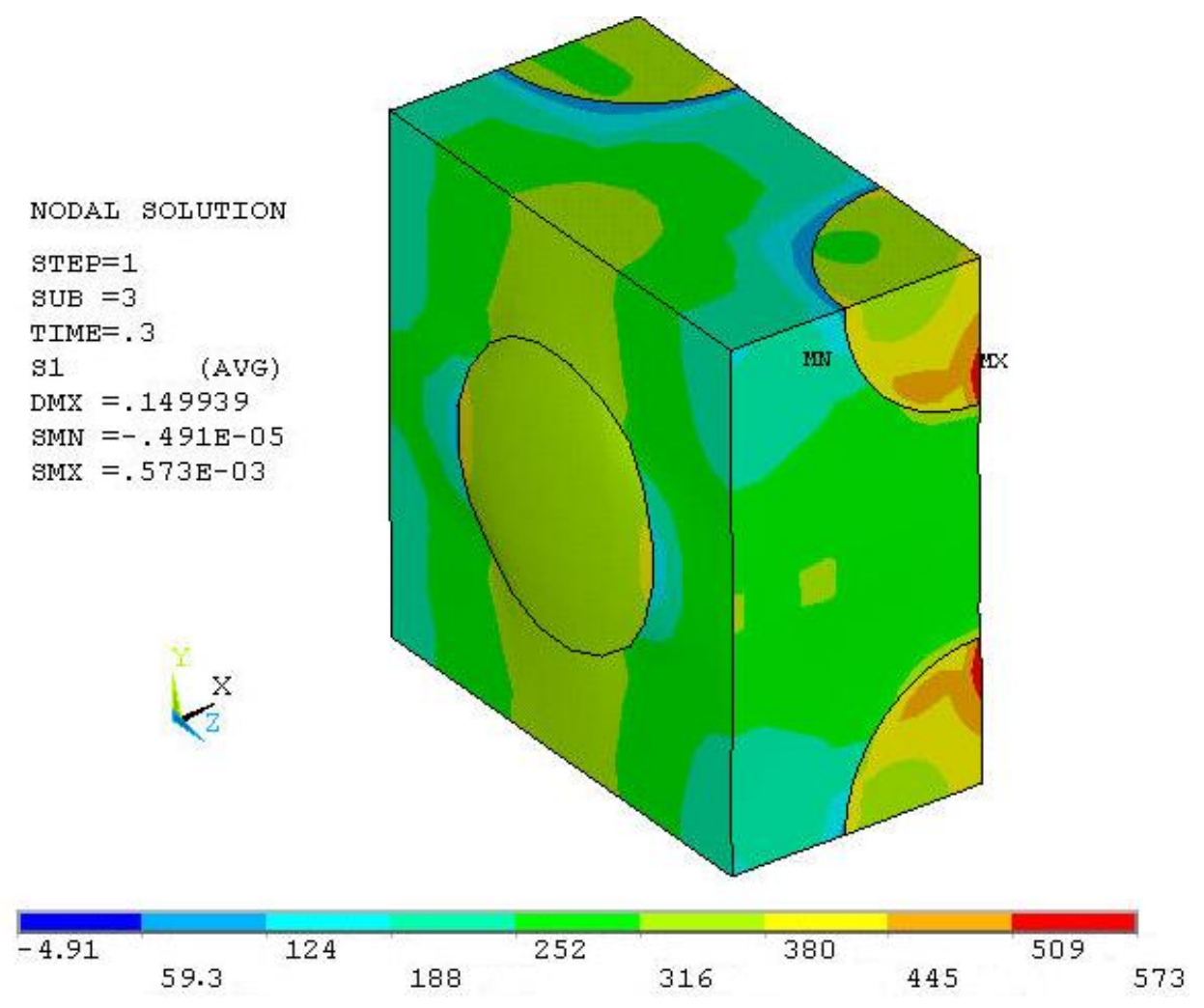

Figure 4.2 - Distribution of maximum principal stress (MPa) in a $20 \%$ volume fraction UCM 
The distribution of von-Mises equivalent stress in the unit cell model with $15 \%$ volume fraction is similar to that of the $20 \%$ unit cell, as can be seen in Figure 4.3.

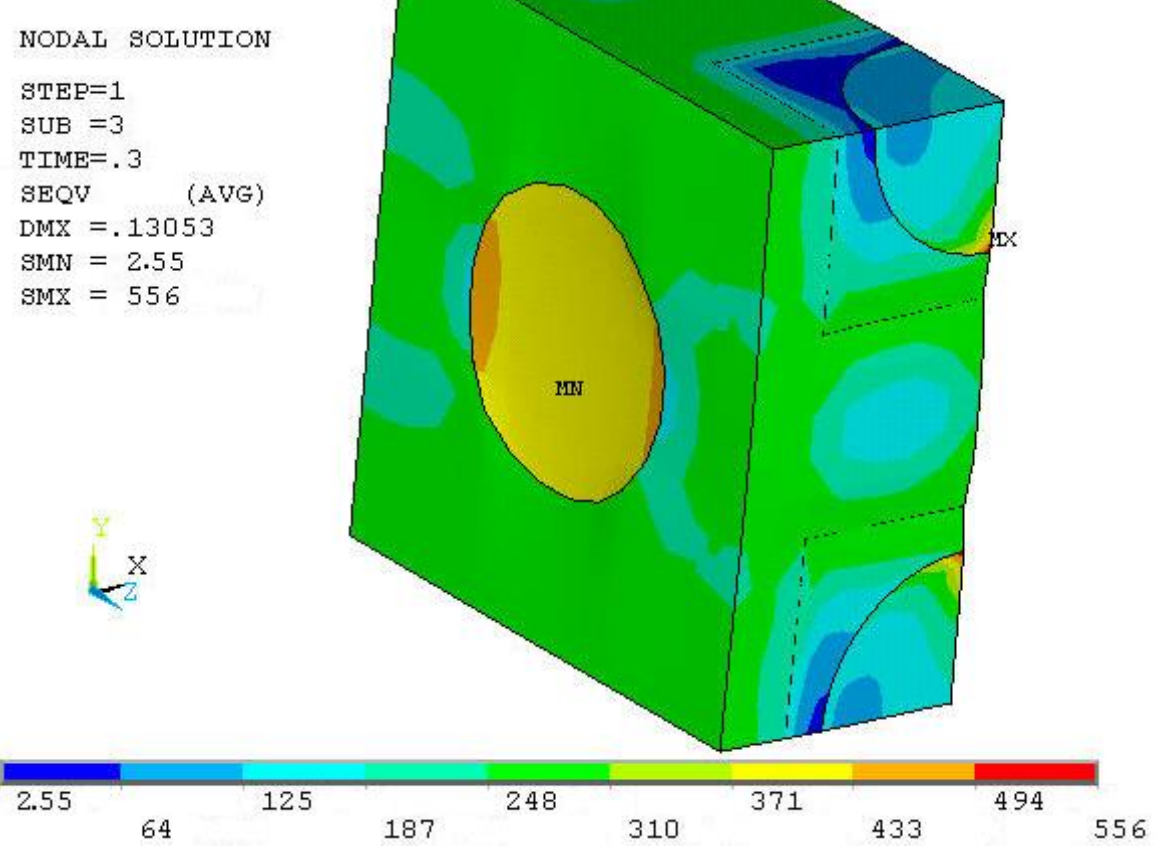

Figure 4.3 - Distribution of von-Mises equivalent stress in 15\% volume fraction UCM.

The distribution of stress shown in Figures 4.3 is captured after the $3^{\text {rd }}$ sub-step of loading, similar to Figures 4.1 and 4.2. The lower amount of stress shown in the $15 \%$ model indicates the lower stiffness of this 15\% MMC. This is evident from Figures 4.4 and 4.5, which compare the von-Mises equivalent strains in UCMs of $15 \%$ and $20 \%$ volume fractions and shows clearly that the $20 \%$ material exhibits less effective strain than the $15 \%$ one.

It is seen that though there are larger regions of high strain in the $20 \% \mathrm{UCM}$, the value of maximum strain is higher in $15 \%$ volume fraction model than the $20 \%$ model. This high strain region occurs in the matrix region of the composite, indicating that the material is likely to fail by matrix yielding. This is the common mode of failure in PRMMCs which have good interface between fiber and matrix, which has been assumed in the current analysis. 


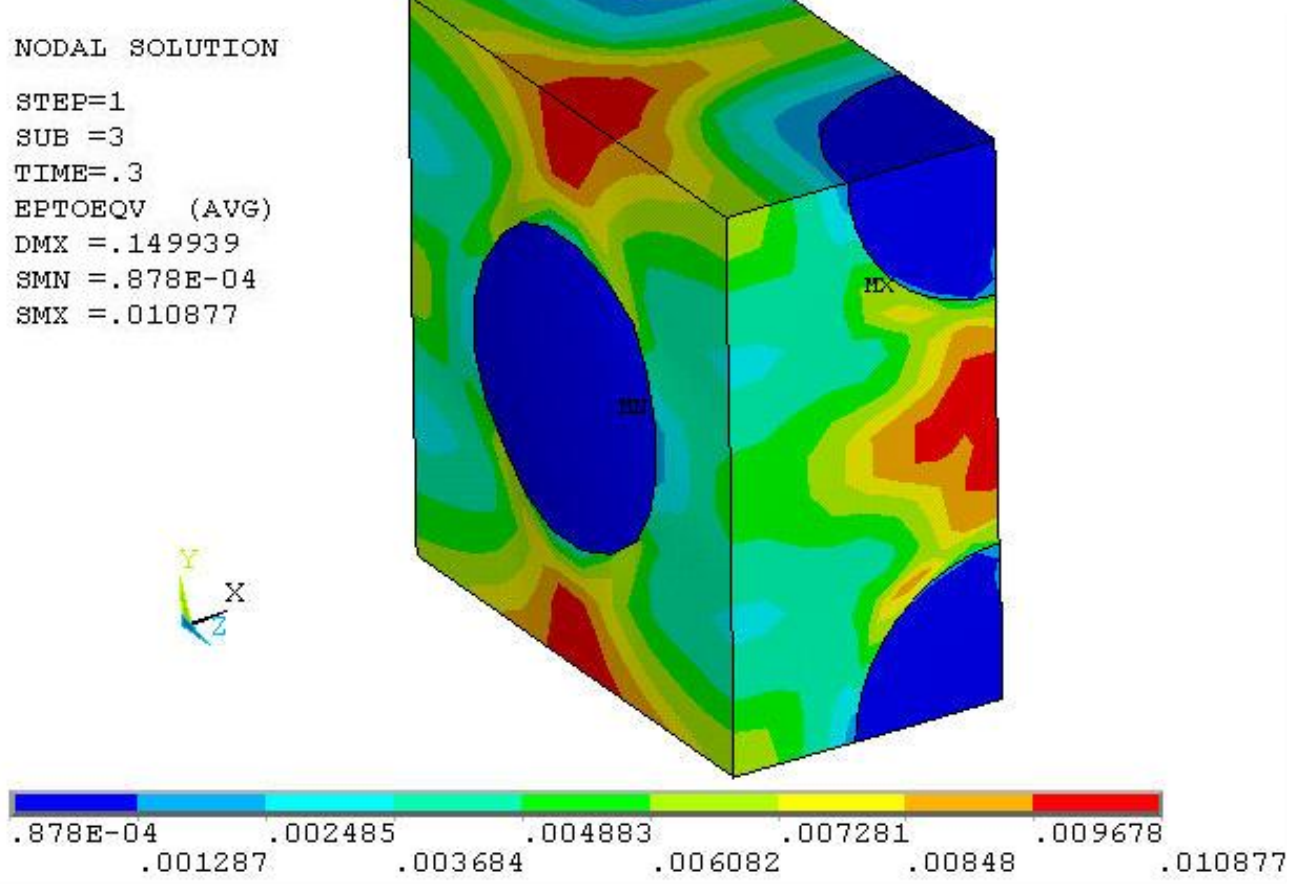

Figure 4. 4 - Distribution of von-Mises equivalent strains in $20 \%$ volume fraction UCM.

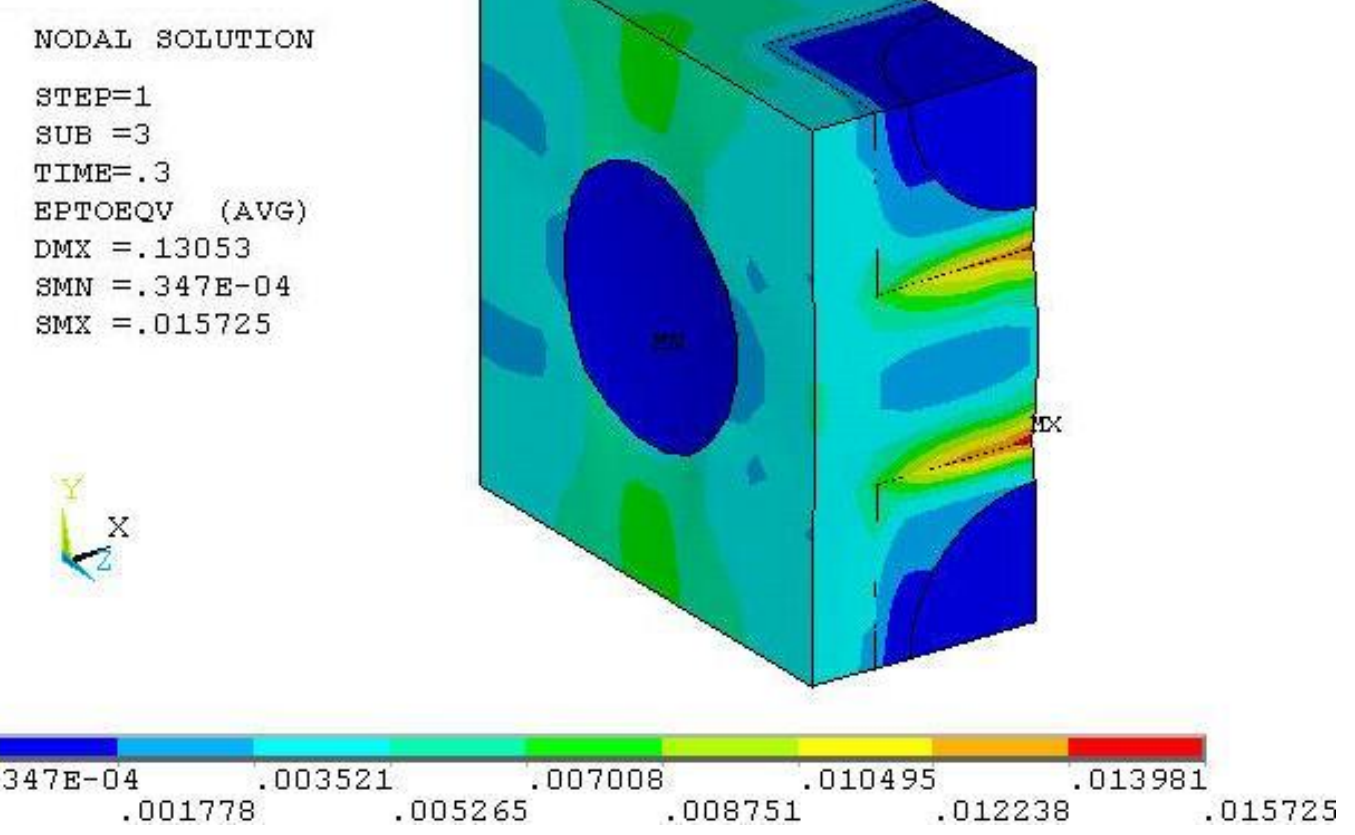

Figure 4.5- Distribution of von-Mises equivalent strains in $15 \%$ volume fraction UCM. 
Due to the boundary conditions imposed on the model and the inherent symmetry of the model itself, the distribution of stresses and strains in completely symmetric. But this type of symmetric distribution is not found in any of the RDM models.

\subsubsection{Spherical RDM}

The distribution patterns in random distribution models vary considerably from UCMs. The von-Mises effective stress in a $15 \%$ spherical inclusion RDM is shown in Figure 4.6. The stress snapshot is taken at a time when the overall stress of the composite is just beyond its yield strength (the average stress in this model is higher than the average stress in the UCM when its snapshot was taken).

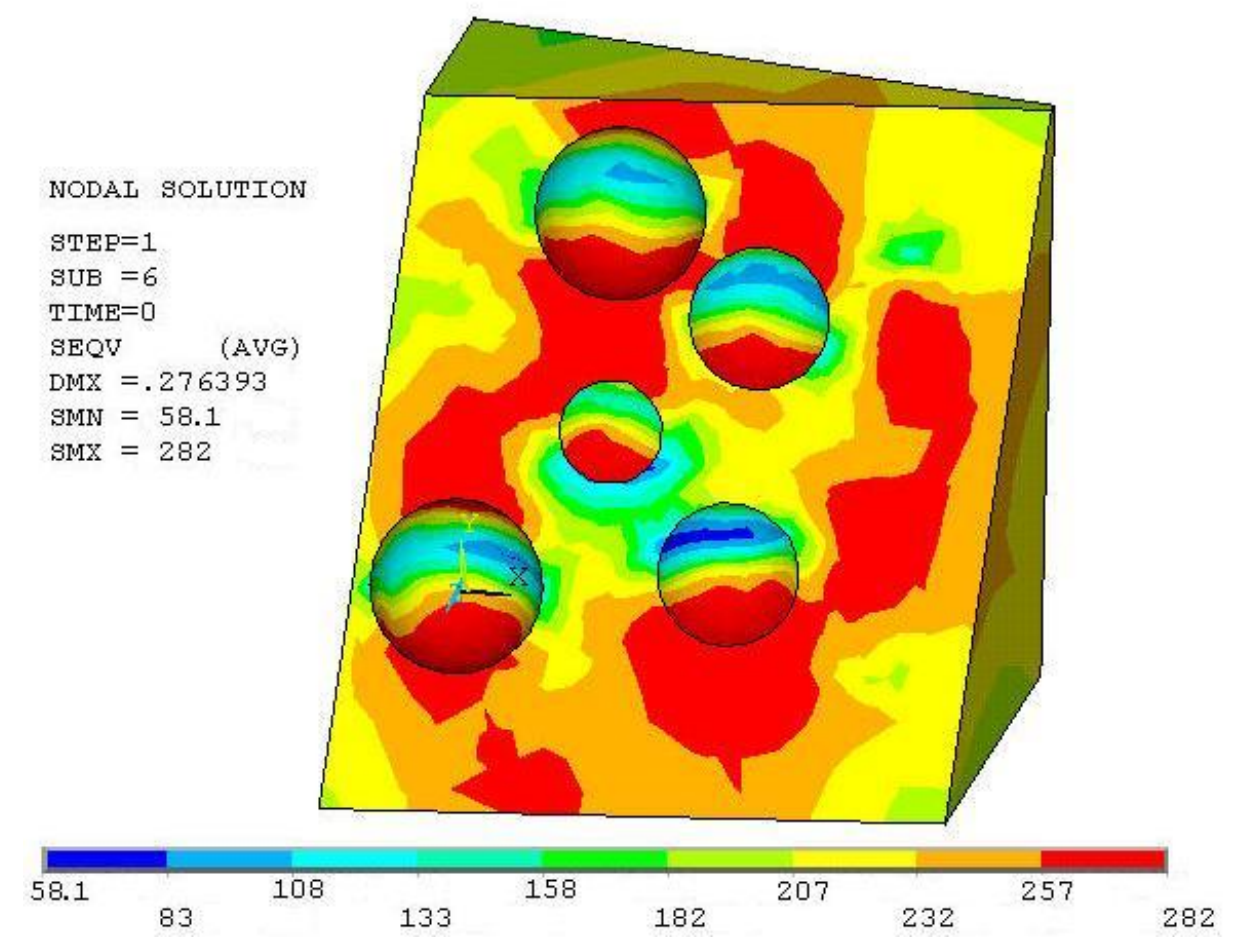

Figure 4.6 - A section of 15\% volume fraction RDM with spherical inclusions, showing the distribution of von-Mises equivalent stress. Note - reinforcing particles are not shown in figure

Figure 5.6 shows the matrix behind the inclusions and the stress distribution in them. It is clear from Figure 5.6 that the maximum stress occurring in the matrix is lesser than those of $15 \%$ UCM, though high stress is more uniformly distributed in the matrix instead of being localized as in the UCMs. Another feature to be noted is the low-stress 
band formed behind every particle in a direction perpendicular to the loading direction. The distribution of maximum principal stress, (shown in Figure 4.7) gives a reason for these low-stress bands. The compressive stress around the particle due to the Poisson's effect gives rise to a low equivalent stress in that area.

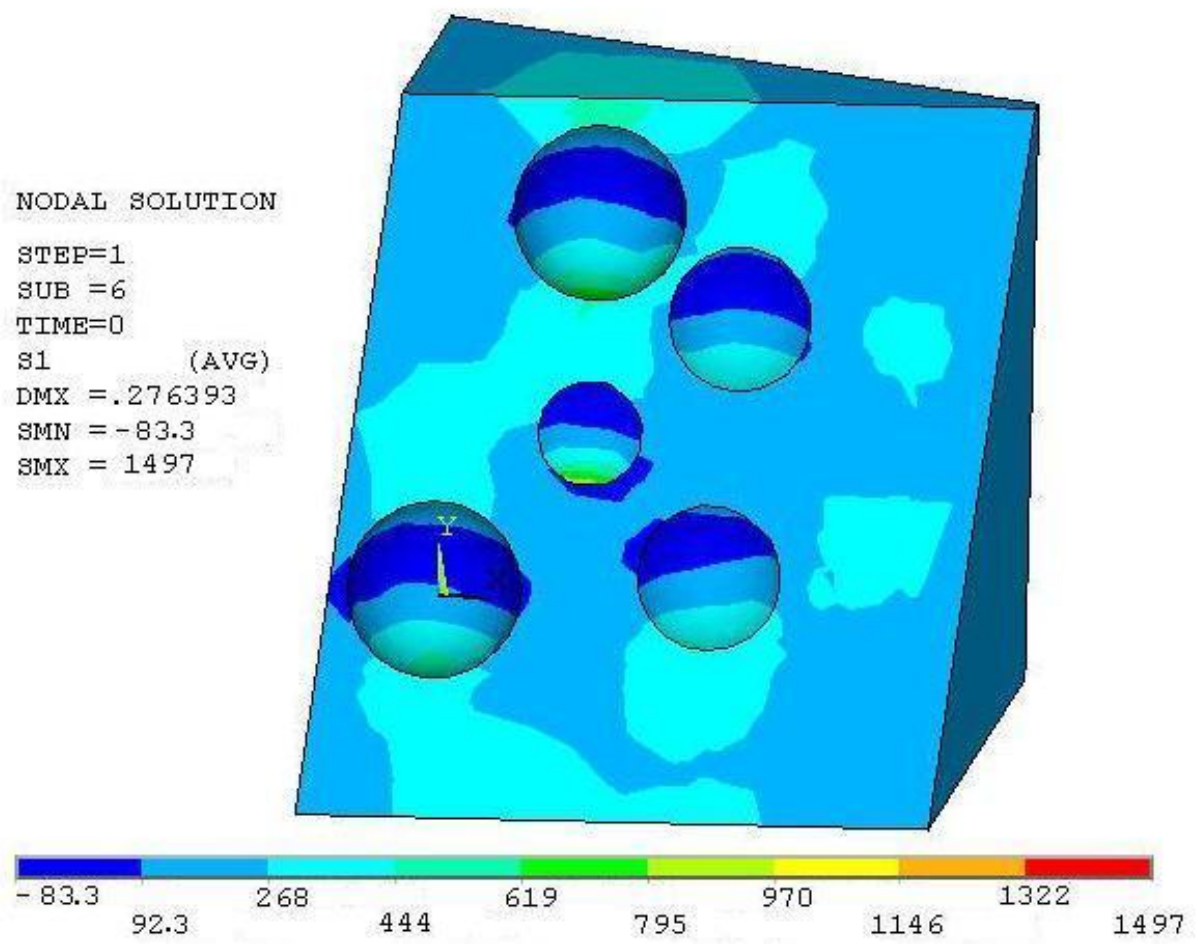

Figure 4.7 - A section of 15\% volume fraction RDM with spherical inclusions, showing the distribution of maximum principal stress.

It is seen that around every particle each low-stress band is accompanied by a high-stress concentration area. These stress concentration areas are especially visible when two particles lie close to each other and it is in these areas that plastic deformation is initiated. So, unlike UCMs where plastic deformation takes place away from particles, RDMs predict that plastic deformation is initiated near the particle-matrix interface. This pattern can be seen clearly in Figures 4.8 and 4.9, which show the strain distribution of RDMs having volume fractions of $15 \%$ and $20 \%$ respectively.

The von-Mises and maximum principal stress distributions for $20 \%$ volume fraction spherical RDM is shown in Figures 4.10 and 4.11. 


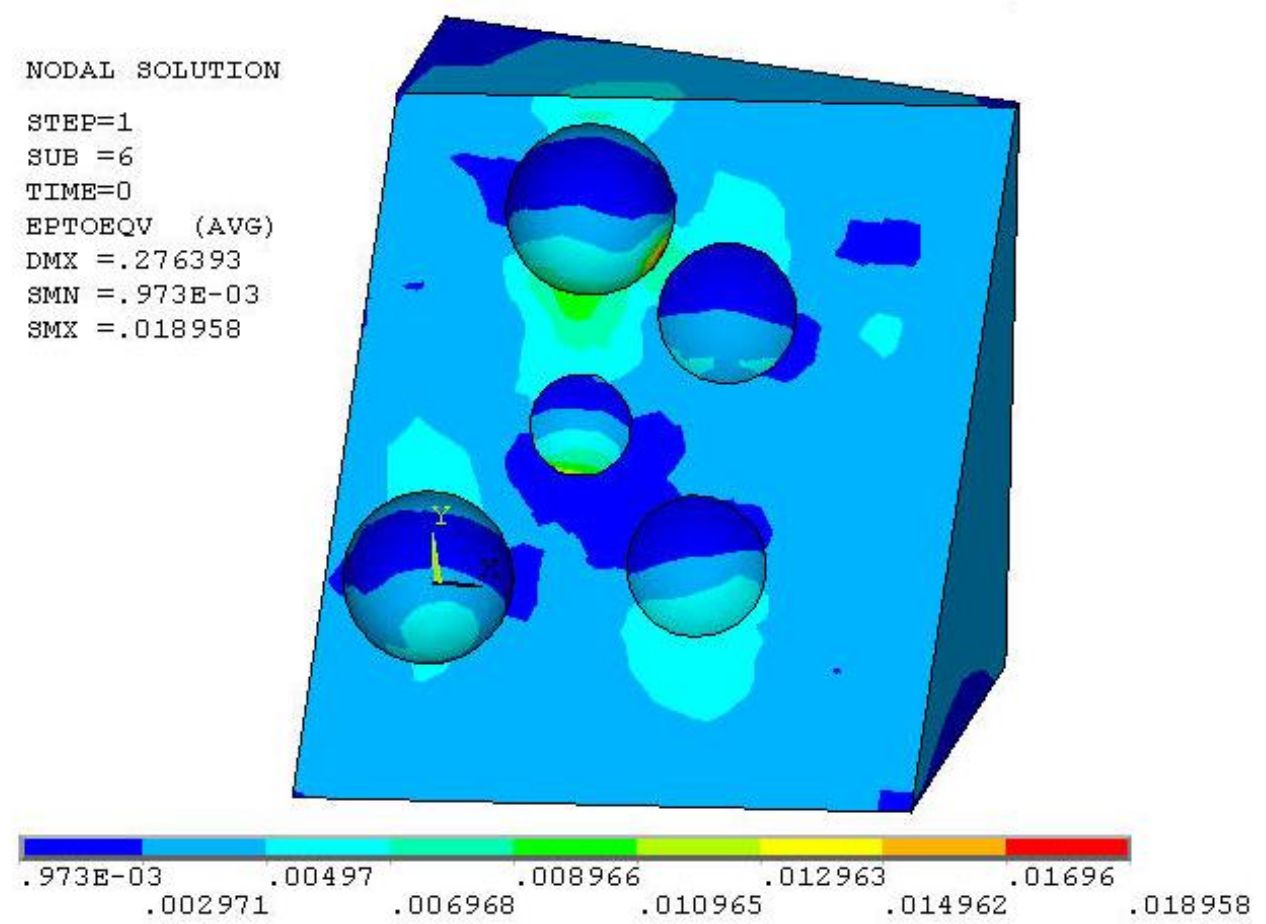

Figure 4.8 - Distribution of von-Mises equivalent strain in the 15\% volume fraction RDM.

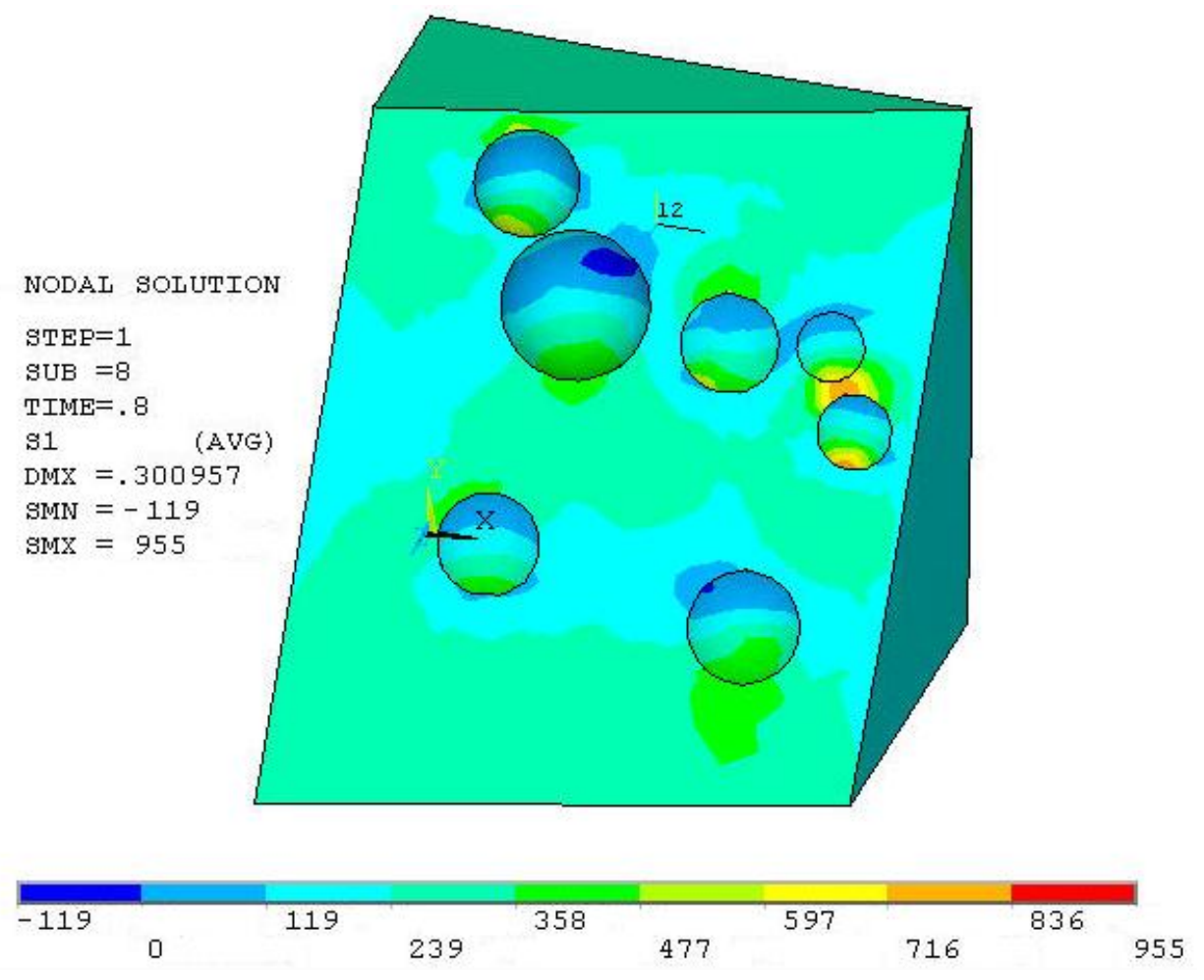

Figure 4.9 - Distribution of the first principal stress in the $20 \%$ volume fraction RDM 


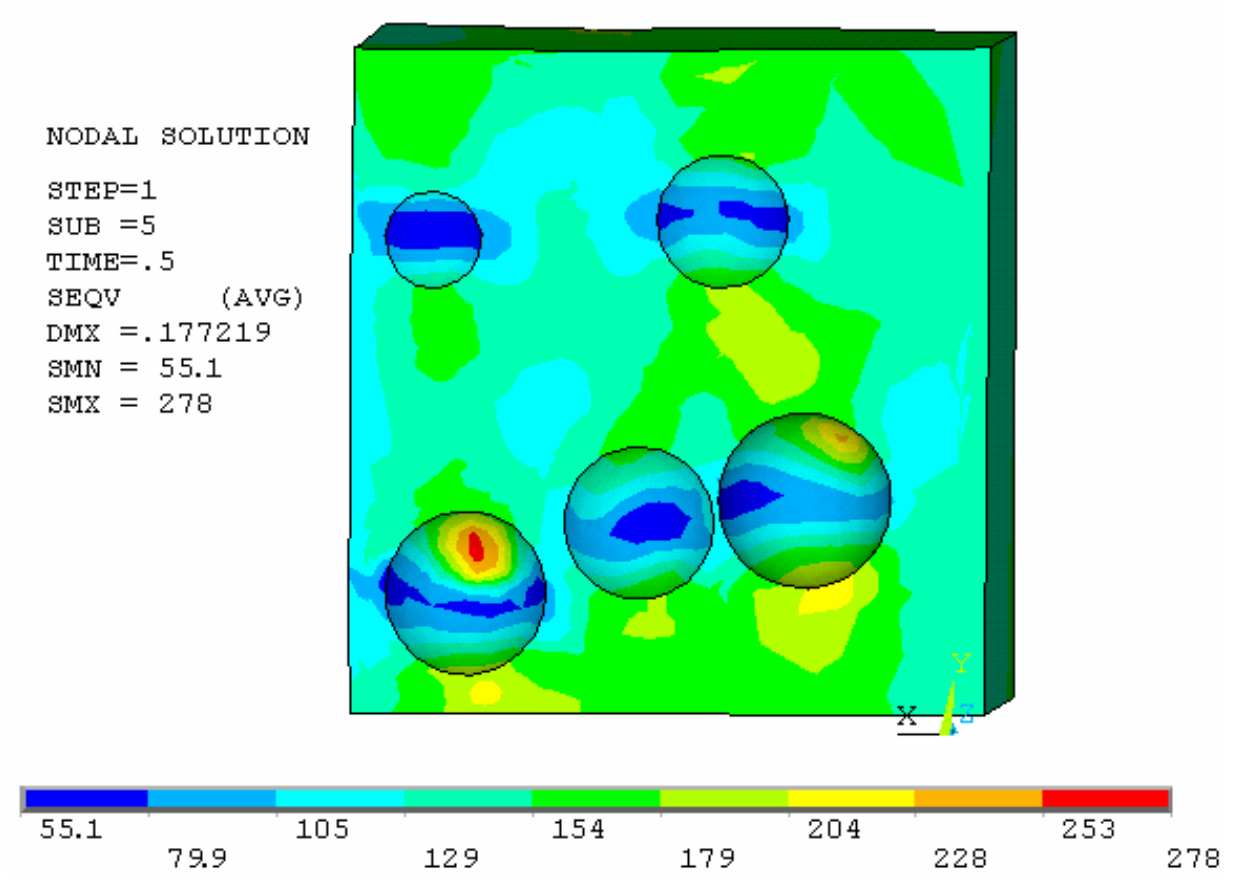

Figure 4.10 - Distribution of von-Mises equivalent stress in a $20 \%$ volume fraction RDM

As in the $15 \%$ reinforcement models, there is a band of low stress in the matrix associated with every particle in the $20 \%$ model. The change in stress levels is shown in Figure 4.11, in which the stresses in the matrix are sampled at various points on the vertical circle around the particle. The plot of these stresses clearly shows two troughs corresponding to the low-stress band and two peaks at the stress-concentrated points. The graph shown is plotted by averaging the stresses around 12 particles. It is also to be noted that the peaks in the principal stresses do not coincide with the peak of von-Mises effective stress, i.e. they are out of phase by a small factor.

\subsubsection{Ellipsoidal RDM}

The distribution of stresses and strains in models having ellipsoidal particles is similar to ones having spherical inclusions except for a few peculiarities. Due to the elongated shape of prolate spheroids, more stress concentration is seen around the inclusions. Another noticeable deviation from the usual distribution pattern is in the orientation low stress bands around the ellipsoids. There are many cases in which the 
low-stress bands are oriented along the major axis of the ellipsoid and not perpendicular to the direction of loading, as can be seen in Figures 4.12-4.13

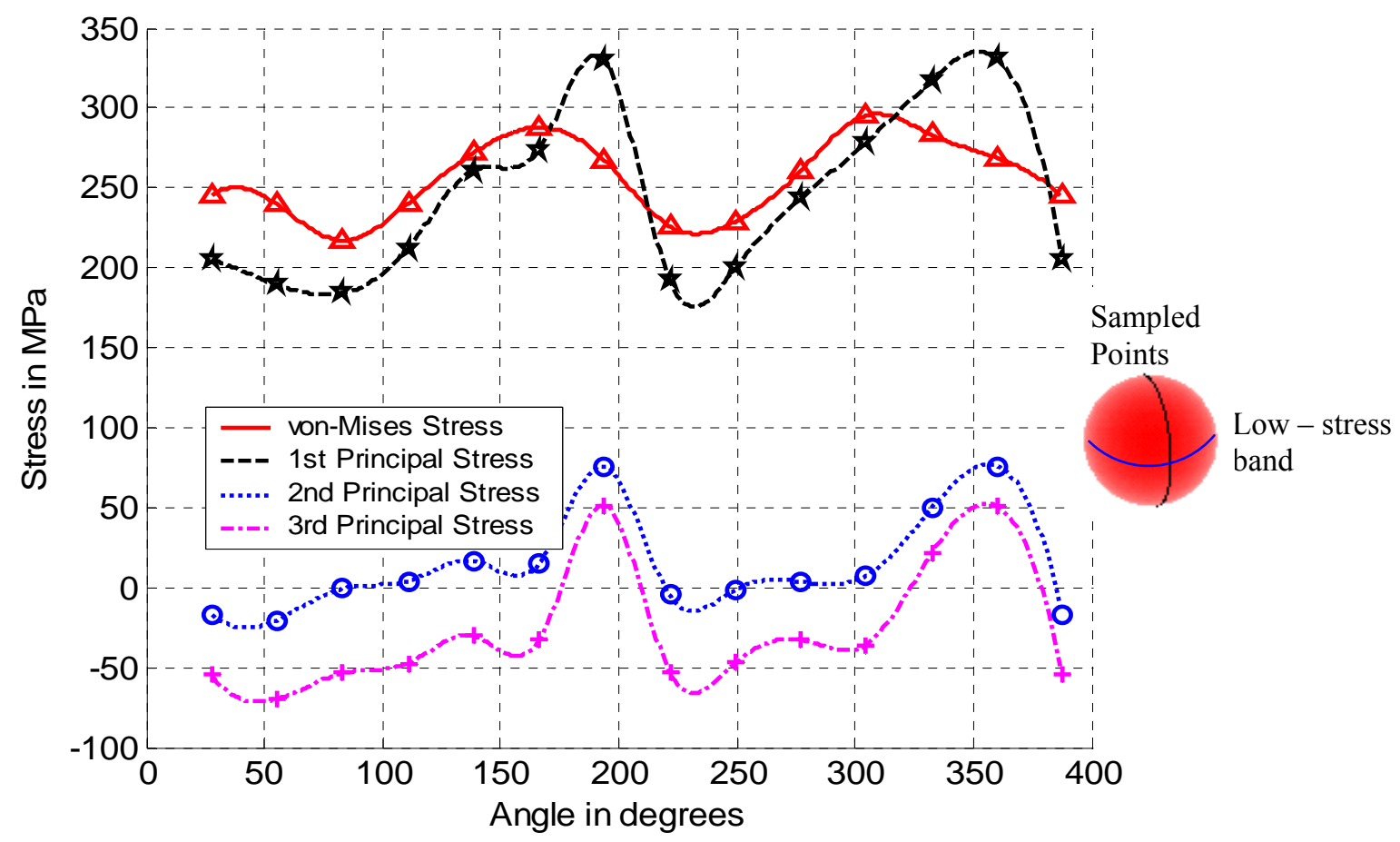

Figure 4.11 - Variation of the von Mises and principal stresses in the matrix around the reinforcement.

One of the effects of the greater stress concentration around the ellipsoids is that yielding is initiated earlier in these sites when compared to models with spherical inclusions. This in turn affects the overall properties of the composite and thus ellipsoidal models predict a lower yield strength than spherical models for any given volume fraction.

This concentration of stresses occurs in the matrix just outside the inclusionmatrix interface. After initiation of yielding at these locations, the progress of failure depends upon the strength of the inclusion-matrix interface. If there is a strong bond between the two phases, the material shows high yield strength and the failure occurs after enough particles have cracked under loading. On the other hand, a weak interface might fail without the reinforcements being fractured, which results in the composite showing low strength. In the present study, a perfect bond between the reinforcement and matrix is assumed. 


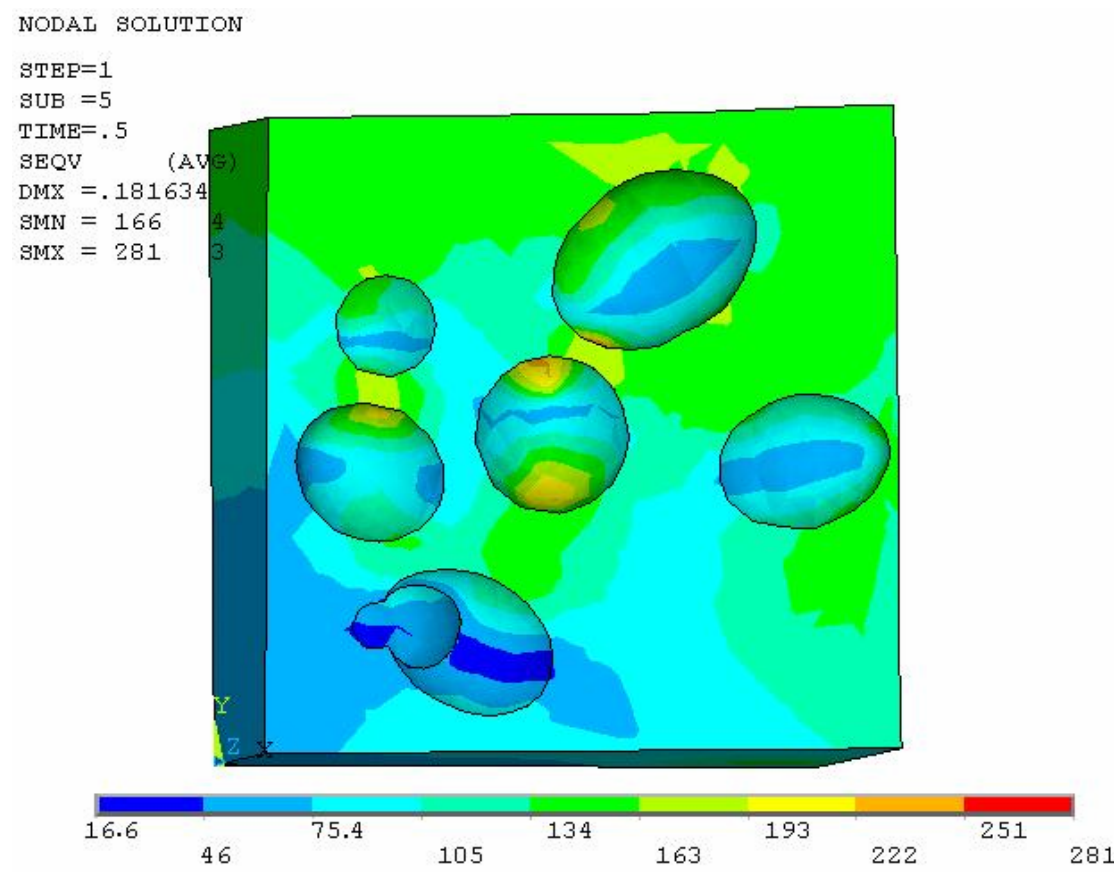

Figure 4.12 - A section through the ellipsoidal RDM model (20\% volume fraction) showing the distribution of von-Mises effective stress.

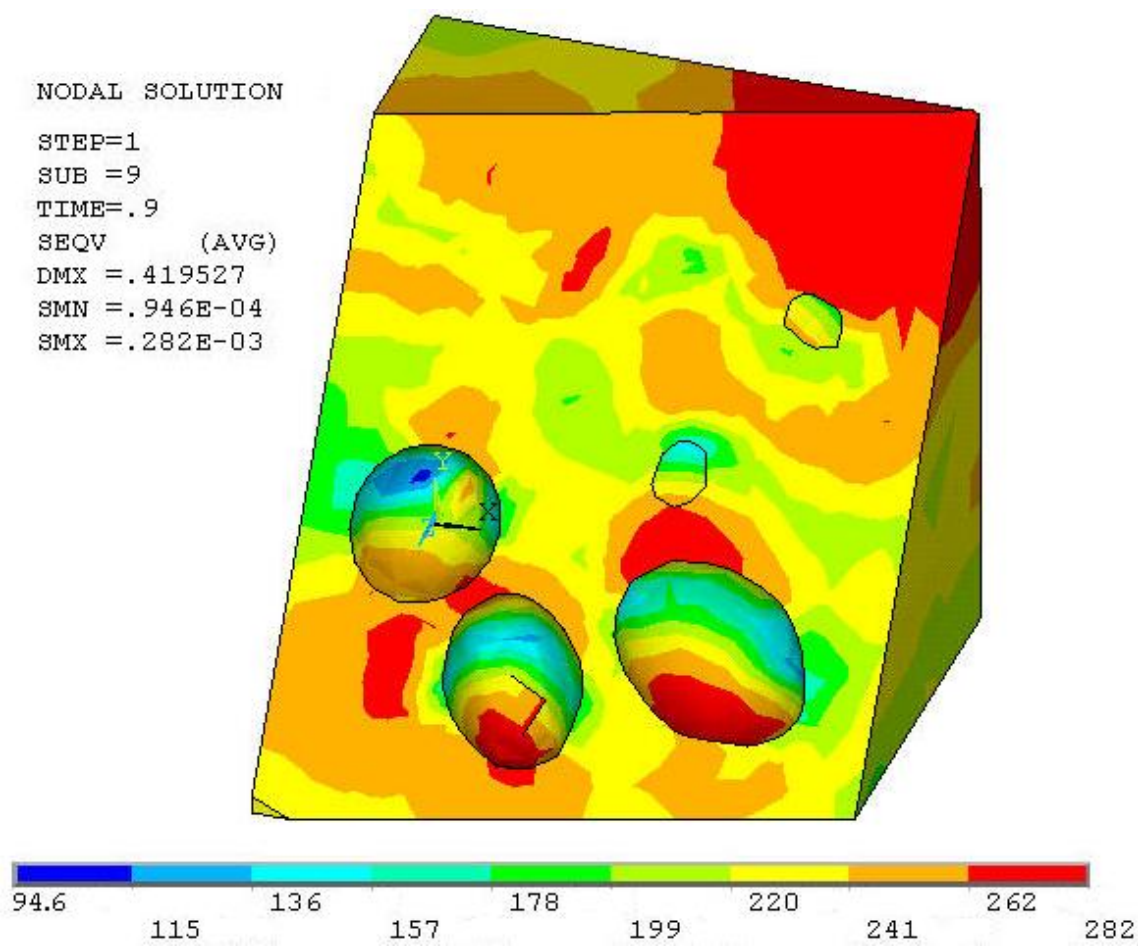

Figure 4.13 - A section through the ellipsoidal RDM model (15\% volume fraction) showing the distribution of von-Mises effective stress. 
Figure 4.14 illustrates the distribution of von-Mises stresses in a typical spherical and ellipsoidal particle embedded in the matrix. Both the particles shown in Figure 4.14 are located at in the middle of the domain analyzed, away from the edges of the cube. Not only is the maximum stress induced in the ellipsoidal particle higher than that in the spherical particle, but also the minimum stress in the ellipsoidal particle is much lower. The ratio of maximum stress to minimum stress is 1.66 for the spherical particle, while it is 2.64 for the ellipsoidal particle.
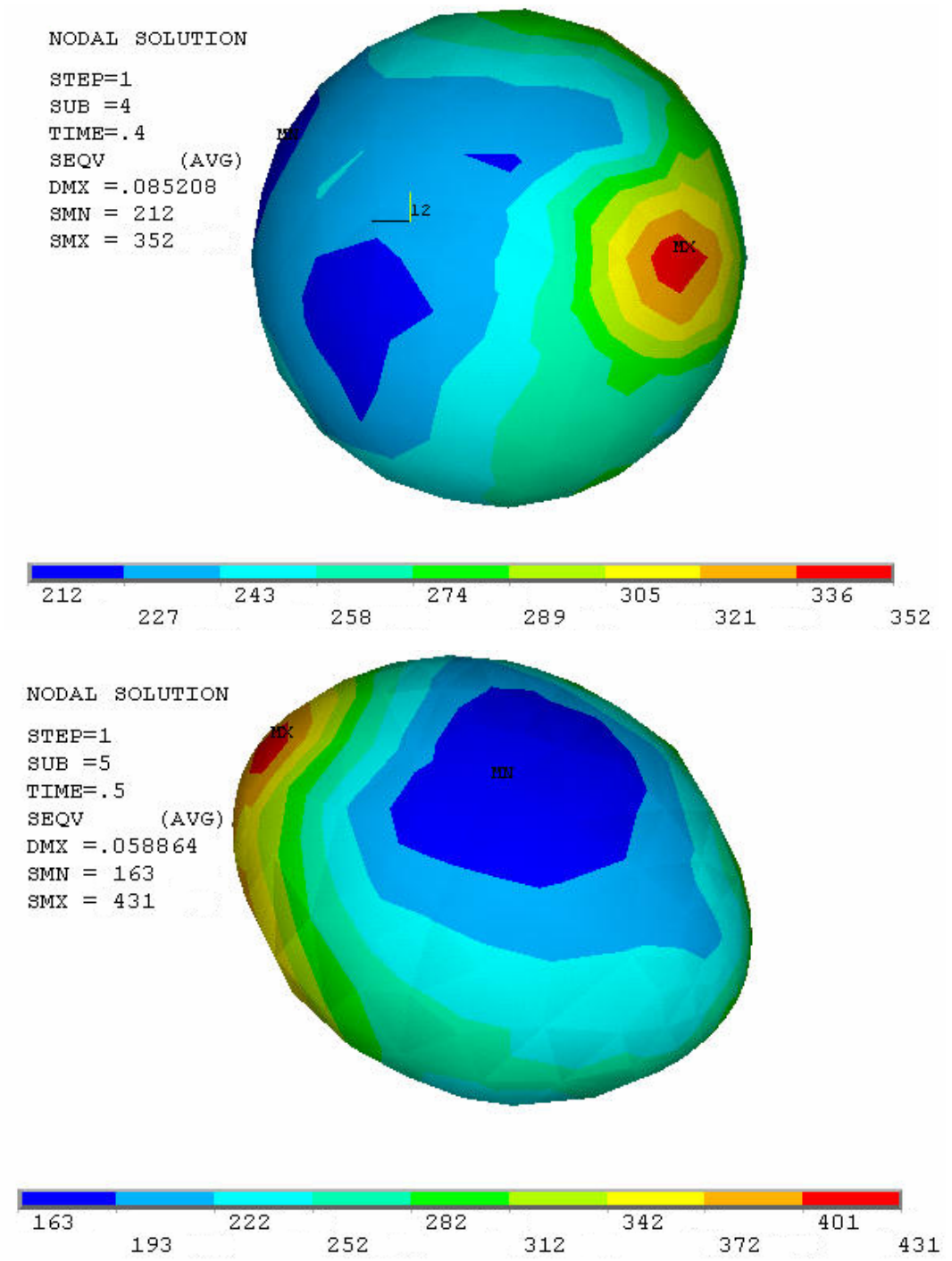

Figure 4.14 - Von-Mises effective stress in a typical spherical and ellipsoidal particle, showing the higher stress concentration induced in the ellipsoidal particle. 


\subsection{Macroscopic Response}

The aim of this study is to determine the overall properties of the MMC. To estimate properties like Young's modulus, tensile strength, tangent modulus, Poisson's ratio etc, the distribution of stresses and strains in microstructure have to re-interpreted with the aim of obtaining average macroscopic properties. Here, a volume weighted average has been used to determine the overall material properties. i.e

$$
\begin{aligned}
& \bar{\varepsilon}_{i j}=\frac{1}{V} \int_{V} \varepsilon_{i j} d V=\frac{1}{V} \sum_{m=1}^{N} \varepsilon_{i j}^{(m)} V^{(m)} \\
& \bar{\sigma}_{i j}=\frac{1}{V} \int_{V} \sigma_{i j} d V=\frac{1}{V} \sum_{m=1}^{N} \sigma_{i j}^{(m)} V^{(m)}
\end{aligned}
$$

where, $\mathrm{V}^{(\mathrm{m})}$ is the volume of element $\mathrm{m}$

$\sigma^{(m)}$ is the stress in element $\mathrm{m}$

$\varepsilon^{(\mathrm{m})}$ is the strain in element $\mathrm{m}$.

$\mathrm{N}$ is the total number of elements in the model

$\sigma_{\mathrm{ij}}, \varepsilon_{\mathrm{ij}}$ are the overall stress and strain in the composite

and $\quad \mathrm{V}$ is the total volume of the cube.

Equations 5.1 and 5.2 are used to estimate the overall stress and strain in the composite at each load sub-step. These stresses and strains are plotted against each other to obtain the overall stress-strain curve of the MMC. The post-processing procedure used to achieve this has been described in chapter three.

The primary aim of all the models was to predict the Young's modulus of the material with good accuracy and in that direction all the models have been successful. By measuring the overall lateral and longitudinal strain in the composite, the Poisson's ratio was computed, which was in turn used to determine the bulk and shear modulii from each model.

Various degrees of anisotropy have been observed in the random distribution models due to the random nature of particle distribution. This anisotropy is evident in the varying Poisson's ratio values in different directions. The predicted stiffness values from 
various models have been summarized in Table 4.1, along with a comparison with the Hashin-Shtrikman ${ }^{[4]}$ and Voigt-Reuss ${ }^{[4]}$ bounds. .

Table 4.1 - Material properties as predicted by the three microstructure models.

\begin{tabular}{|c|c|c|c|c|c|c|}
\hline & $\begin{array}{c}\text { Volume } \\
\text { fraction }\end{array}$ & $\begin{array}{c}\text { Ellipsoidal } \\
\boldsymbol{R D M}\end{array}$ & $\begin{array}{c}\text { Spherical } \\
\boldsymbol{R D M}\end{array}$ & BCC UCM & $\begin{array}{c}\text { Hashin- } \\
\text { Shtrikman } \\
\text { bounds }\end{array}$ & $\begin{array}{c}\text { Voigt- } \\
\text { Reuss } \\
\text { bounds }\end{array}$ \\
\hline $\mathbf{E}$ & $15 \%$ & 89.2 & 90.5 & 83.2 & $89.8-106.1$ & $82.15-122.7$ \\
$\mathbf{( G P a )}$ & $20 \%$ & 95.2 & 97.8 & 91 & $96.67-118$ & $86.21-126$ \\
& $30 \%$ & - & 119.2 & - & $112.2-144$ & $95.65-173.4$ \\
\hline & $15 \%$ & $0.313-0.32$ & $0.329-0.331$ & 0.322 & $0.30-0.32$ & $0.274-0.301$ \\
$\boldsymbol{v}$ & $20 \%$ & $0.30-0.31$ & $0.304-0.312$ & 0.318 & $0.29-0.31$ & $0.259-0.292$ \\
& $30 \%$ & - & $0.287-0.293$ & - & $0.27-0.30$ & $0.234-0.273$ \\
\hline $\mathbf{G}$ & $15 \%$ & 33.79 & 34.02 & 31.47 & $33.93-40.71$ & $32.2-47.16$ \\
$\mathbf{( G P a )}$ & $20 \%$ & 36.48 & 37.39 & 34.52 & $36.64-45.66$ & $34.24-48.76$ \\
& $30 \%$ & - & 46.20 & - & $42.94-56.65$ & $38.76-68.11$ \\
\hline \multirow{2}{*}{$\mathbf{K}$} & $15 \%$ & 80.36 & 88.73 & 77.90 & $84.5-89.36$ & $60.58-102.8$ \\
$\mathbf{( G P a )}$ & $20 \%$ & 81.37 & 84.90 & 83.33 & $88.31-94.46$ & $59.62-101.0$ \\
& $30 \%$ & - & 94.60 & - & $96.44-104.97$ & $59.93-127.3$ \\
\hline
\end{tabular}

The prediction of the Young's modulus and Poisson's ratio from the UCM deviates slightly from the experimentally determined value and the H-S bounds. This is due to the stress distribution caused form the unrealistic boundary conditions applied on the model to achieve periodicity of the microstructure. It is seen that the values predicted from the RDM models are within or close to the H-S and V-R bounds, serving as further validation for the models. The variation of stiffness of the material with volume fraction along with the H-S and V-R bounds is plotted in Figure 4.15. While the BCC unit cell model's predictions fall outside the H-S bounds, the spherical and ellipsoidal RDM's prediction of Young's modulus is very close to the H-S lower bound.

In the post-processing phase, the overall stress and strain at each sub-step of the applied load was calculated and written to an ASCII file. These values for a composite having 20\% volume fraction are plotted in Figure 4.16. The reported values for the $20 \%$ composite are obtained from [32] and [33]. 


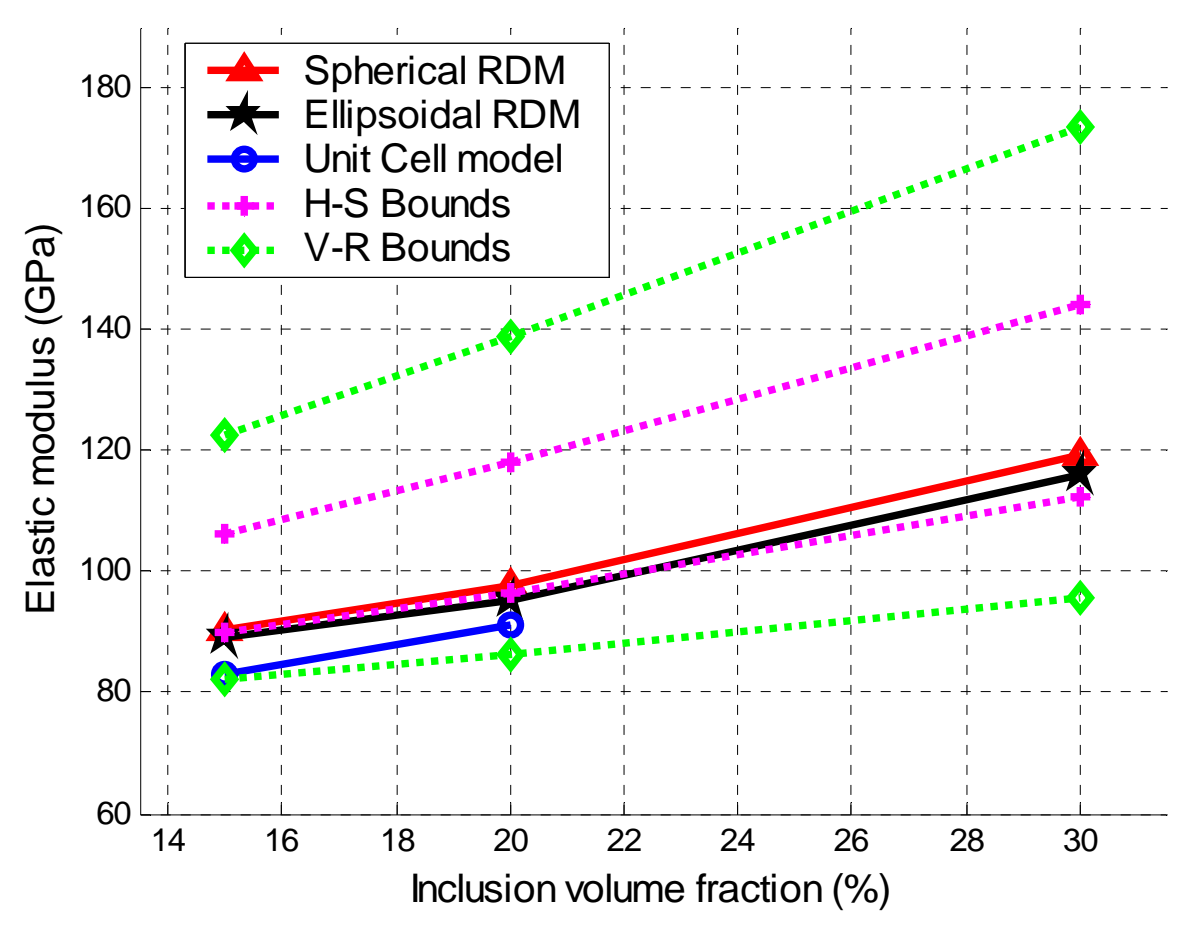

Figure 4.15 - The variation of Young's modulus of the composite with the volume fraction.

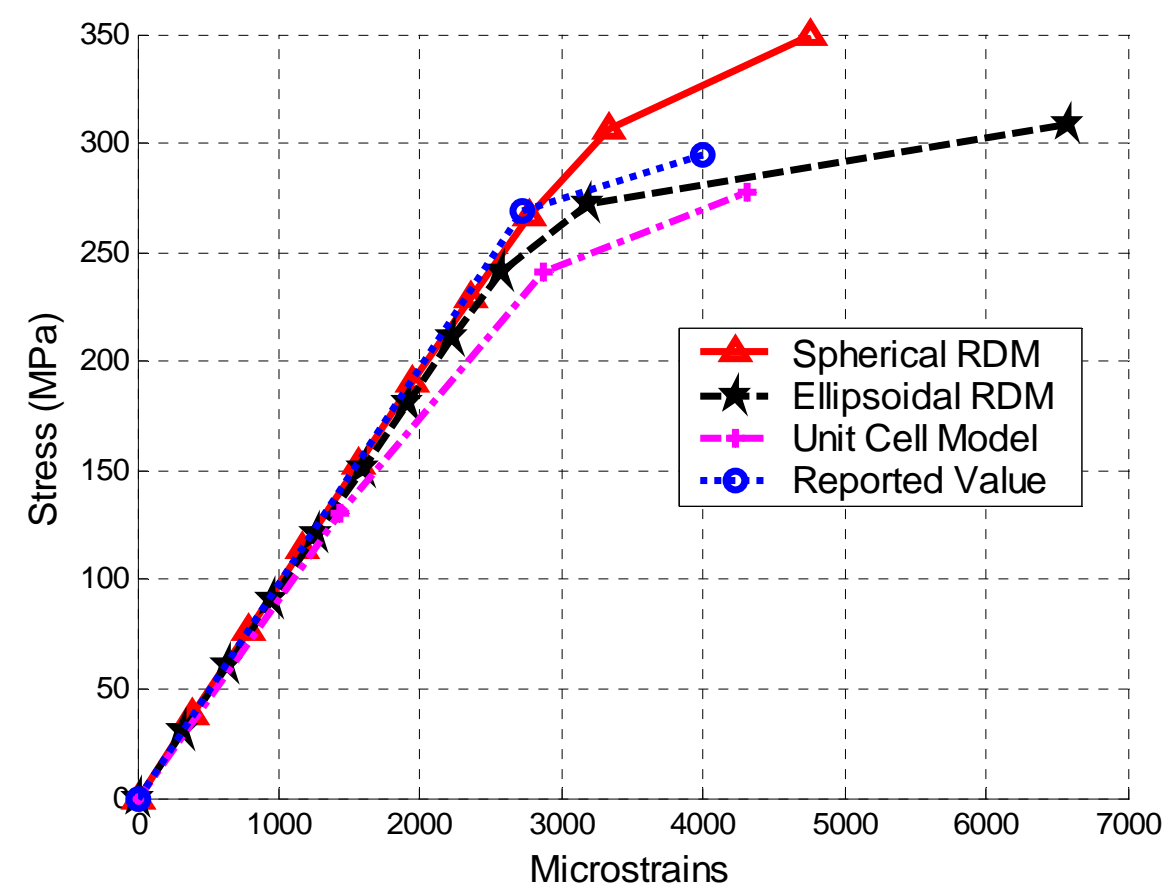

Figure 4.16 - The predicted and reported stress-strain curves for a $20 \%$ volume fraction MMC.

The above response is obtained from a BCC model having a cube length of $30 \mu \mathrm{m}$ and $8.63 \mu \mathrm{m}$ inclusion radius. Both, the spherical and ellipsoidal RDM models have a 
cube length of $70 \mu \mathrm{m}$ and average particle size of $10 \mu \mathrm{m}$. It is seen that the RDM containing ellipsoids has lower yield strength than the model containing spherical inclusions. This early onset of yielding in the ellipsoidal model is due to the higher stress concentration at the tip of the ellipsoidal particles. And it is due to the same reason that the Young's modulus prediction of the ellipsoidal model is slightly lower than that of the spherical inclusion model. It is to be noted that the periodic array model predicts a much lower yield point than the reported value.

The original intention of validating the FE models using experimental test data was not possible because of the differences in material tested and the assumptions made in the FE model. The specimens for testing were prepared from cutting a brick of MMC on the EDM. Further, the specimens were not subjected to any heat-treatment. Heat treating the specimen induces tensile stresses in the matrix and highly compressive stresses in and around the particles, due to the difference in the co-efficient of thermal expansion between the two phases ${ }^{[54]}$. A lack of heat treatment results in the formation of lower residual stress in the composite, which is equivalent to having an imperfect bond between the matrix and the reinforcement. This condition contradicts the assumption of an ideal interface between the matrix and reinforcement made in the FE models. Due to the weak interface and lack of residual stresses in the tested specimen, they displayed a low value of tensile strength. Though results from testing have been used to validate the Young's modulus predicted by the FE model, for strength validation, values from the literature had to be made use of. Figure 4.17 shows the stress-strain curve of $20 \% \mathrm{MMC}$ obtained from testing, as compared to the value from other numerical models.

For the material having $15 \% \mathrm{SiC}$ reinforcement, the trend of predictions is the same as what is seen in the $20 \%$ material, though the values vary, as can been from Figure 4.18. Unfortunately, no reported values for $15 \% \mathrm{SiC}$ volume fraction material were found in the literature to make a comparison with the FE values. 


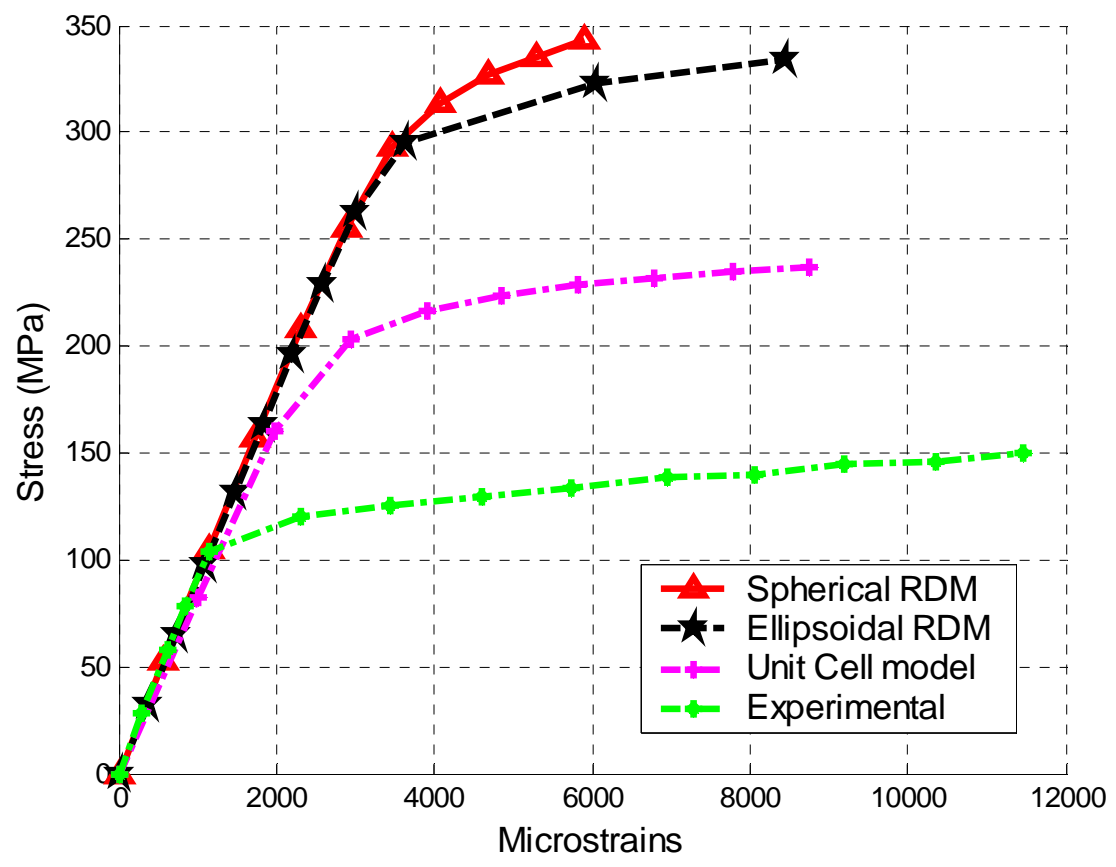

Figure 4.17 - Stress-strain curves for a 15\% volume fraction MMC as predicted by various FE models, compared with the experimental values.

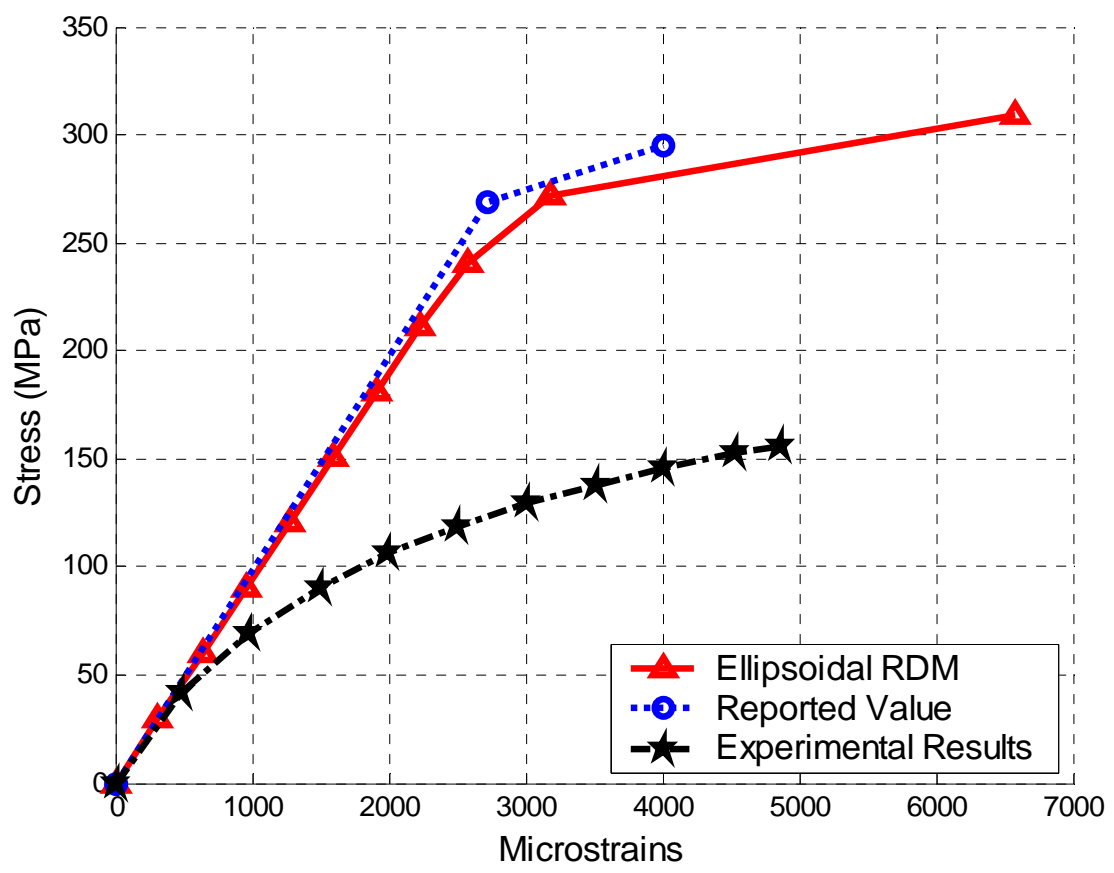

Figure 4.18 - Comparison of values obtained for $20 \% \mathrm{SiC}$ material from ellipsoidal RDM model and experiments. 
As the values from experimental testing could not be used to validate the accuracy of models, various material systems which have been investigated by other researchers were used to test the accuracy of the modeling technique. Table 4.2 gives a comparison of the $0.2 \%$ yield strength of various material systems and their experimentally obtained values.

Table $4.2-0.2 \%$ yield strength as predicted by the two models and their experimentally determined values

\begin{tabular}{c|ccc}
\hline Material & $\begin{array}{c}\text { Ellipsoidal RDM } \\
(\mathrm{GPa})\end{array}$ & $\begin{array}{c}\text { Spehrical RDM } \\
(\mathrm{GPa})\end{array}$ & Reported (GPa) \\
\hline $\mathrm{A} 356 / \mathrm{SiC}_{\mathrm{p}}-20$ & 290 & 370 & $331^{[53]}$ \\
$\mathrm{Al} 6061 / \mathrm{SiC}_{\mathrm{p}}-25$ & 385 & 437 & $407^{[54]}$ \\
$\mathrm{A} 16061 / \mathrm{SiC}_{\mathrm{p}}-15$ & 338 & 345 & $397^{[55]}$ \\
$\mathrm{A} 16061 / \mathrm{SiC}_{\mathrm{p}}-20$ & 295 & 366 & $345^{[56]}$ \\
$\mathrm{A} 12014 / \mathrm{Al}_{2} \mathrm{O}_{3}-15$ & 427 & 492 & $470^{[57]}$ \\
\hline
\end{tabular}

It is seen from the table above that the values predicted by the two modeling techniques are in reasonable agreement with the experimentally determined values. The high degree of accuracy is maintained even when different reinforcement system like alumina particles are used. 


\section{Chapter Five \\ 5 Mesoscale modeling of MMC material}

\subsection{Mesoscale Modeling}

Modeling the MMC as a periodic array of unit cells is an over-simplification of the microstructure, which assumes that every single cell behaves in an identical manner. In reality, local variations in the volume and area fractions are observed from one region to other. These variations are observed in scales higher than the size of the inclusion and their effect on the overall response of the composite should not be neglected. In this regard, a mesoscale model was developed to address the effect of particle clustering in the microstructure of the MMC. Arrays of finite elements are used to simulate particle clusters by assigning random material properties to each of the element in the array. An important aspect of this model is the degree of particle clustering, which has to be determined from analyzing the micrograph of the material to determine the average volume fraction and the standard deviation in volume fraction in various regions.

The microstructure models described previously were generated for volume fractions varying from $5 \%$ to $35 \%$ at intervals of $5 \%$. The output of these models provides a family of stress-strain curves for various volume fractions. These curves are used as input to the mesoscale model. The variation in the volume fraction at various regions of the material is assumed to follow normal distribution, with the mean located at the overall volume fraction of the material. Figure 5.1 shows the probability density function for a normal distribution with the mean located at zero and standard deviation of 1. Similarly, based on a probability distribution function generated from user defined standard deviation and mean, different elements in the mesoscale model are randomly assigned to be of different volume fractions while keeping the overall volume fraction of the entire model at the specified mean. 


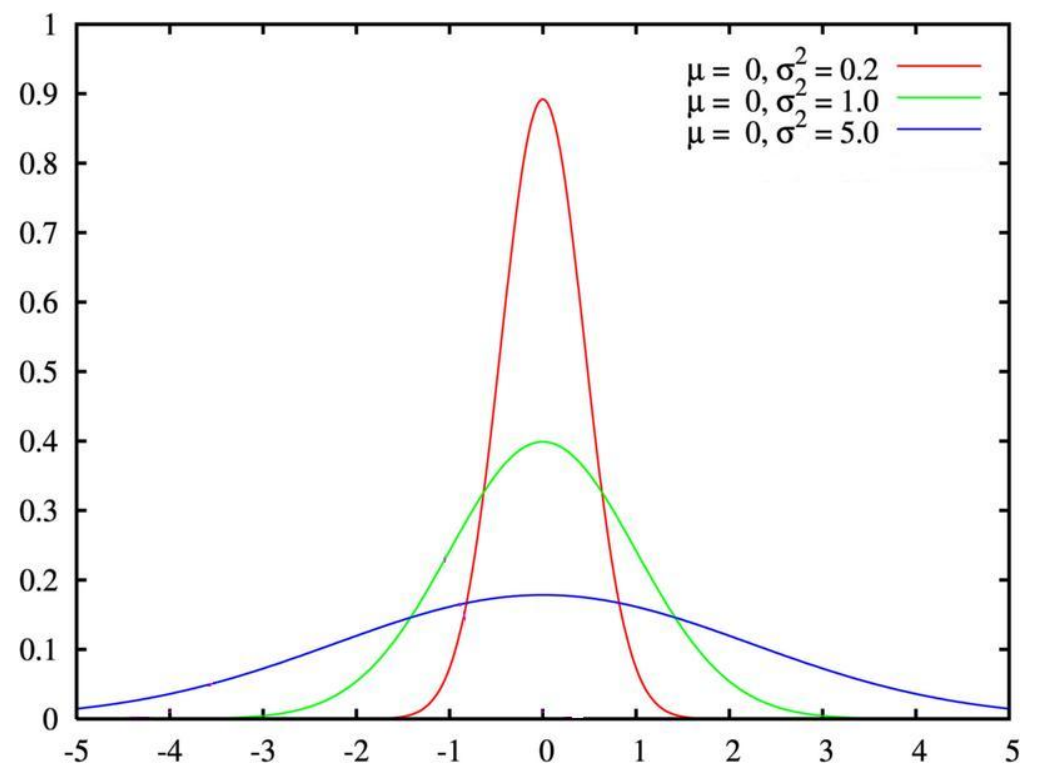

Figure 5.1 - Probability density function for normal distribution having $\mu=0$

Nine materials are defined as having volume fractions from $0 \%$ to $40 \%$ at $5 \%$ intervals. A cube of MMC material having a length of $0.5 \mathrm{~mm}$ is considered and meshed with 8000 8-noded solid elements. A Matlab routine which uses normally distributed random numbers and takes the overall volume fraction and standard deviation as input is used to assign each element with one of the above defined 9 materials in such a way that the overall volume fraction of the model is maintained at the specified level. When the generated random volume fractions fall outside the available materials, the following rules are used:

$$
\begin{array}{ll}
0<v f \leq 2.5 & v f=0 \\
2.5<v f \leq 7.5 & v f=5 \\
7.5<v f \leq 12.5 & v f=10 \\
12.5<v f \leq 17.5 & v f=15 \\
17.5<v f \leq 22.5 & v f=20 \\
22.5<v f \leq 27.5 & v f=25 \\
27.5<v f \leq 32.5 & v f=30 \\
32.5<v f \leq 37.5 & v f=35 \\
37.5<v f & v f=40
\end{array}
$$

where $\mathrm{vf}$ is the randomly generated volume fraction.

By increasing the standard deviation of the particle cluster volume fraction in the mesoscale model, a clustered microstructure having regions of all volume fractions is 
generated. Figure 5.2 shows the number of elements assigned with various volume fractions in an 8000 element, $20 \%$ overall volume fraction mesoscale model. Figure 5.2(a) is a model with 5\% Std.D in volume fraction and it can be seen that most of the elements are assigned as having $20 \%$ volume fraction, depicting a mostly uniform microstructure. Figure 5.2(b) is a material with $10 \% \mathrm{SD}$ in volume fraction, and so, the number of $15 \%$ and $25 \%$ elements is comparable to the number of $20 \%$ elements, with a few $0 \%$ and $40 \%$ elements also being present. When the standard deviation is increased to a high value like $20 \%$ as shown in Figure 5.2(c), the microstructure is assignment almost only $0 \%$ or $40 \%$ materials, depicting a highly clustered material having only particle rich and particle poor regions.
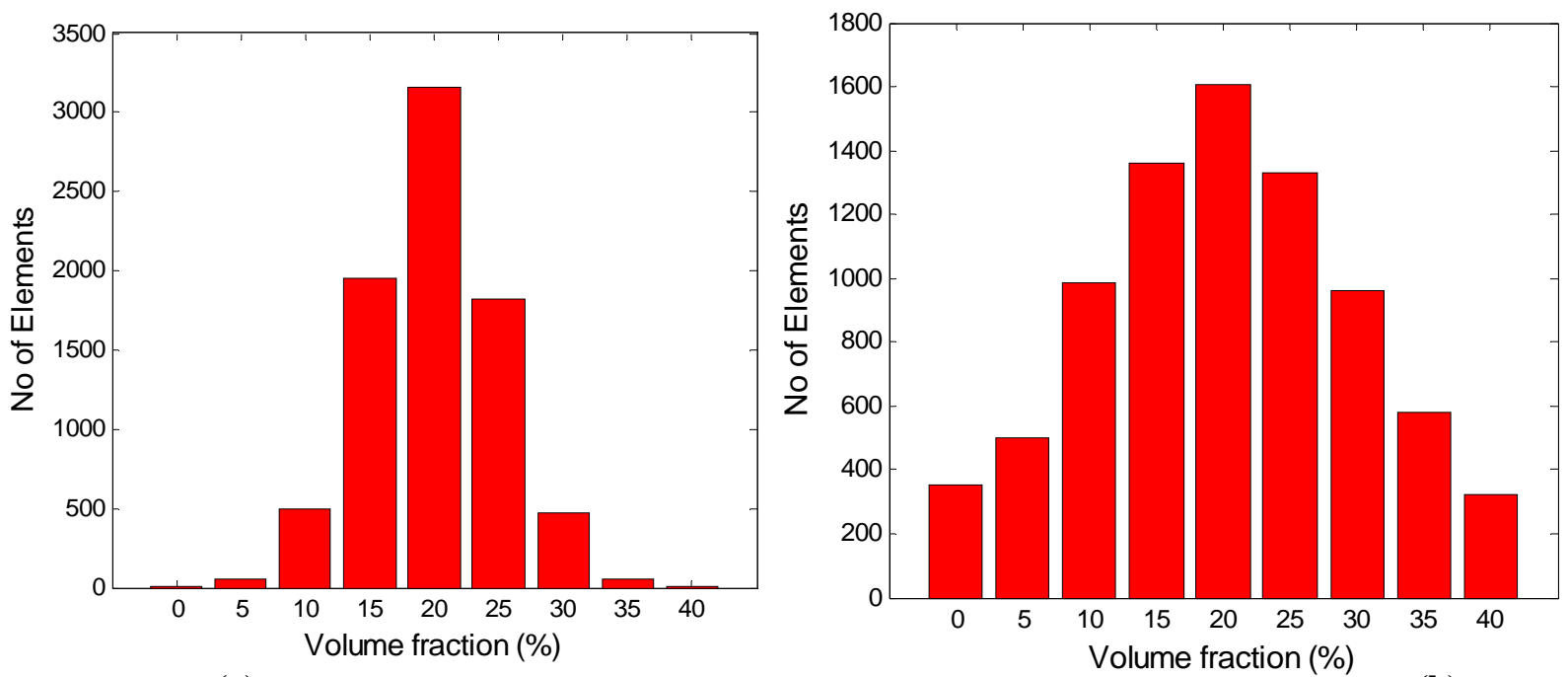

(a)

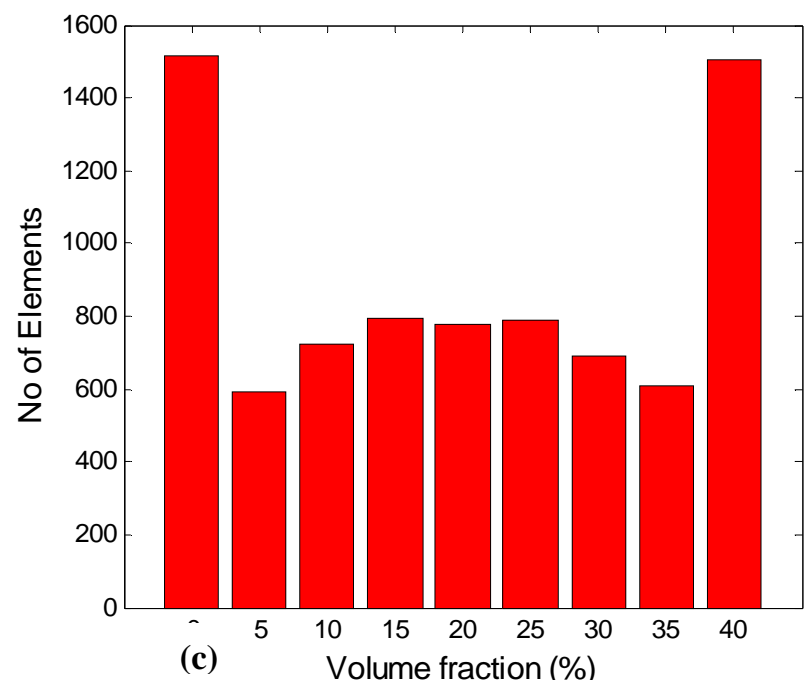

Figure 5.2 - The number of elements having various volume fractions in an 8000 element mesoscale model 
Though no models were generated having $40 \%$ volume fraction, their stress-strain curves were approximated by extrapolating the data obtained from other, lower volume fraction models. An example model having an overall volume fraction of $20 \%$ and a standard deviation of $10 \%$ is shown in Figure 5.3.

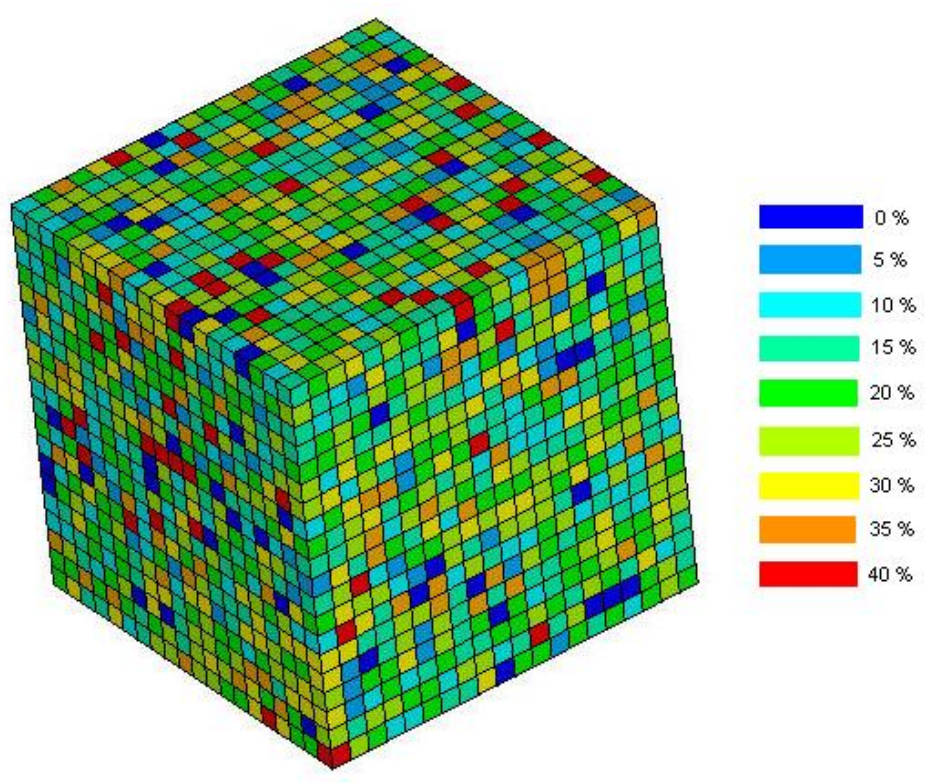

Figure 5.3 - Mesoscale random array with $20 \%$ volume fraction and 0.1 SD.

Similar to the model in Figure 5.3, models were generated with volume fractions of $15 \%$ and $20 \%$ and varying degrees of clustering. Models generated in this manner are constrained in the same manner as the RDM RVEs - the three faces that meet at the origin are defined with symmetry boundary condition. The loading is applied in the form of displacement of $0.01 \mathrm{a}$ at $\mathrm{x}=\mathrm{a}$ face, where $\mathrm{a}$ is the length of the cube. The overall properties of the cube are calculated using the same rule as for the RDM models, by using a volume weighted average of the stresses in each element to determine the overall stresses.

\subsection{Results}

Neither the ellipsoidal nor the spherical models by themselves come close to the reported stress-strain curve of $20 \% \mathrm{Al}-/ \mathrm{SiC}$. The mesoscale model, by incorporating the particle clustering effect comes closer than either of the RDMs to the real stress-strain 
curve. The response of a random array model, with its materials defined from the family of ellipsoidal particles and having a volume fraction of $20 \%$ and standard deviation of $10 \%$ in volume fraction is shown in Figure 5.4.

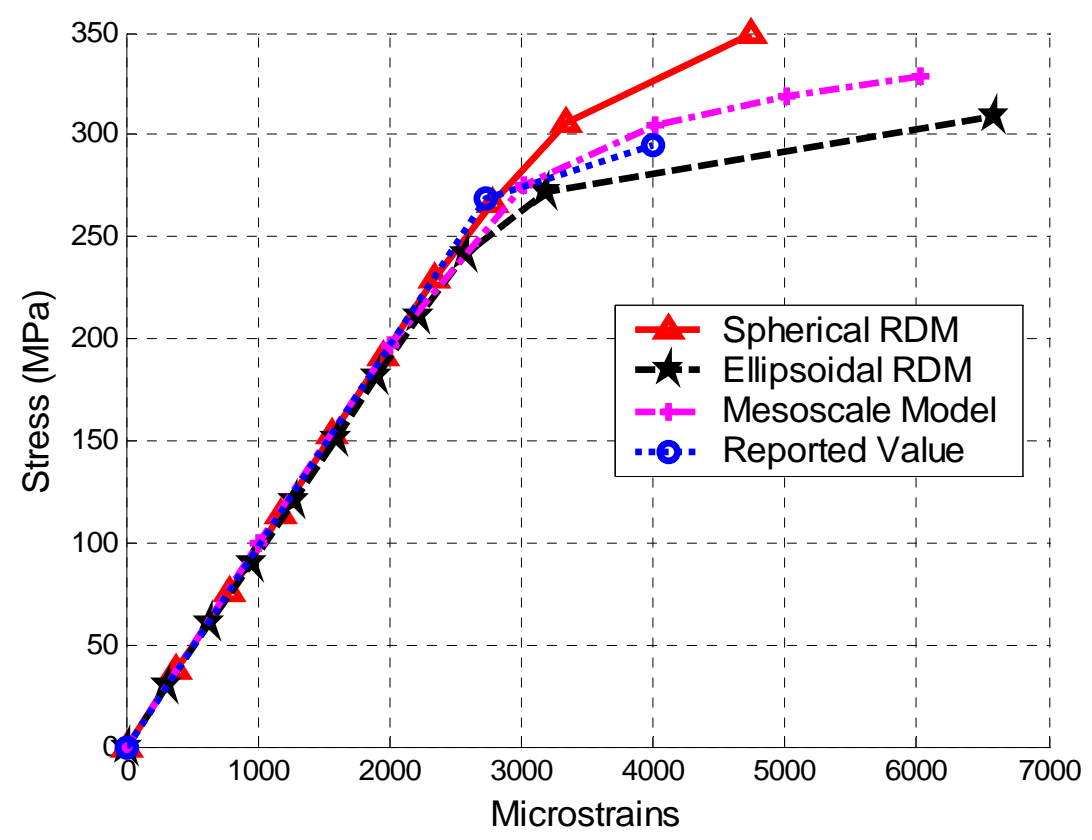

Figure 5.4 - Stress-strain curve from the random array mesoscale model and the reported curve in the literature for $20 \%$ volume fraction material.

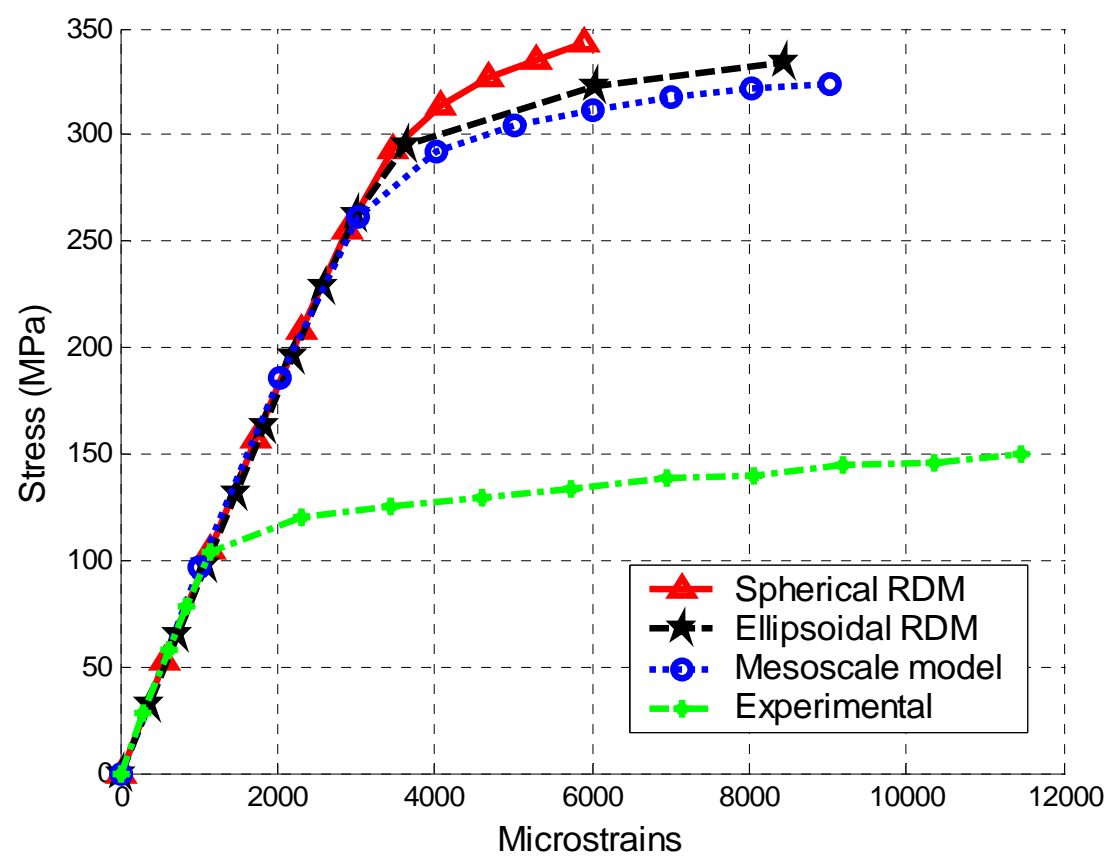

Figure 5.5 - Stress-strain curve from experiments and numerical models for $15 \%$ volume fraction material. 
Figure 5.5 gives a summary of predictions by various models and the experimental results for $15 \%$ volume fraction material. The only change in the trend is by the ellipsoidal model, which shows a higher tensile strength than the mesoscale model. This is due to the smaller number of particles present in the model, resulting in a lower concentration of stresses than in the $20 \%$ model. This lower stress concentration pushes up the overall tensile strength of the material. Also, due to the lower volume fraction, the experimental specimens tested showed a higher strain to failure than the $20 \%$ specimens.

\subsubsection{Effect of Particle Clustering}

One of the important characteristics of $\mathrm{MMC}$ microstructures in particle clustering, which is heavily dependent on the fabrication method and conditions during processing. Particle clustering also affects the overall material properties to a great extent through a combination of effects such as void nucleation, stress concentration, dislocation density etc. The aim of the mesoscale model developed in this study was to effectively model the clustering of particles using statistical features of the microstructure. Using this technique, the effect of highly clustered and lightly clustered microstructures can be modeled.

To measure the effect of particle clusters on material behavior, four mesoscale models all having an overall volume fraction of $20 \%$, but with varying standard deviations in local volume fraction $(5 \%, 10 \%, 15 \%$ and $20 \%)$ were analyzed. Figure 5.6 shows the stress-strain curves of the material as predicted by these models. 


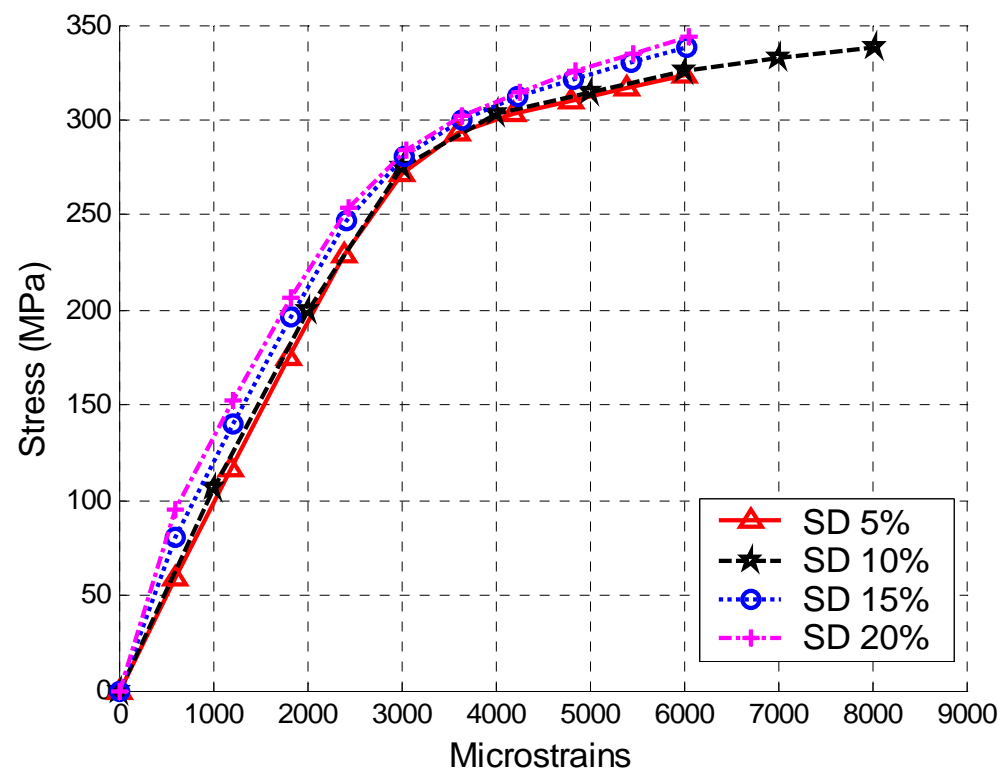

Figure 5.6 - Stress-strain curves from mesoscale models having different standard deviations of local volume fractions.

The increase in standard deviation increases the number of elements having high volume fractions, which initially increases the stiffness of the material. Though the yield point of all the models nearly coincides, the tangent modulus of clustered microstructures is high. This behavior has been observed by previous researchers including Bruzzi et al ${ }^{[1]}$. But experimental investigations have shown that highly clustered microstructures show lower yield strength than uniform ones, due to void nucleation and lower matrix reinforcement bonding in the clustered regions. Since this study does not take into consideration the bond strength between the reinforcement and matrix, this effect could not be captured in the results of the finite element model.

It is seen from the preceding discussion that the results from the FE models agree reasonably with the reported values in literature. Thus, these values can be used in real life applications where MMCs are intended for use. One such example is provided in the next chapter, which deals with designing bolted joints made of MMC material and uses results from microstructure modeling as input to the FE models of bolted joints. 


\section{Chapter Six}

\section{Experimental testing and FE modeling of MMC bolted joints}

\subsection{Introduction}

One of the advantages of PRMMC material for their use in mainstream industry is their ability to be connected using bolted joints. For fiber-reinforced MMCs bolted-joint connections are not suitable due to the difficulty in drilling holes, which causes fiber misalignment and loss of strength at the joint. Welding is ruled out for any composite member as the high temperatures make the two phases to chemically react with each other. But in case of particulate reinforced metal matrix composites, bolted joint connections are a viable option. Bolted joints offer many advantages over conventional adhesion techniques: surface treatment is not a requirement, rapid assembly and disassembly is possible, and thick members can be joined efficiently.

Though considerable research has gone into the field of bolted joints, little has been reported on MMC bolted joints. This study presents theoretical and experimental approach to investigate the stress distributions around the hole in a bolted joint made of particulate metal matrix composite. A three-dimensional finite element parametric model has been developed to study the effects of various design parameters on the structural performance of such joints. All the bolted joints considered in this chapter are made of $20 \% \mathrm{SiC}$ volume fraction MMC.

\subsection{Experimental evaluation}

The experimental plan was the test various double lap joints made of MMC material with varying joint parameters like edge distance, joint size etc to investigate their effect on the strength of the joint. The MMC material investigated earlier in the study, having aluminum as the matrix and silicon carbide ( $\mathrm{SiC})$ as reinforcing particles was used to prepare the bolted joint test specimen. The values obtained from the test results are 
compared with EUROCODE standards and with results from finite element modeling of joints.

For standard bolted joint connections, it is long known that the e/d and w/d ratios play a major part in determining the strength of the joint.

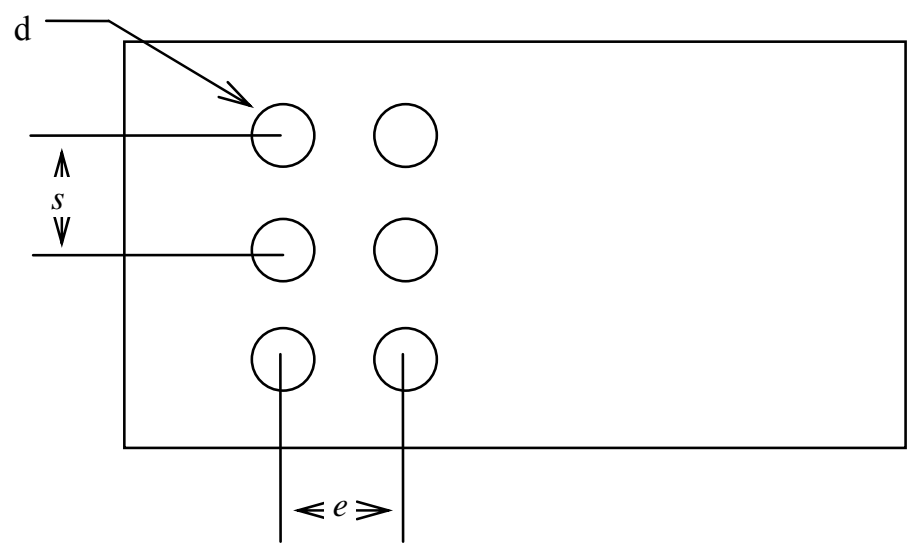

Figure 6.1 - A standard bolted joint shown with the parameters which are used to describe the joint.

In the current investigation, 5 kinds of specimens having varying e/d and w/d ratios are tested to determine their effect on the strength of the bolted joint. Three values of edge distance are used (0.6", 1.5", 2.5") and two values of w (1.5", 2") are used.
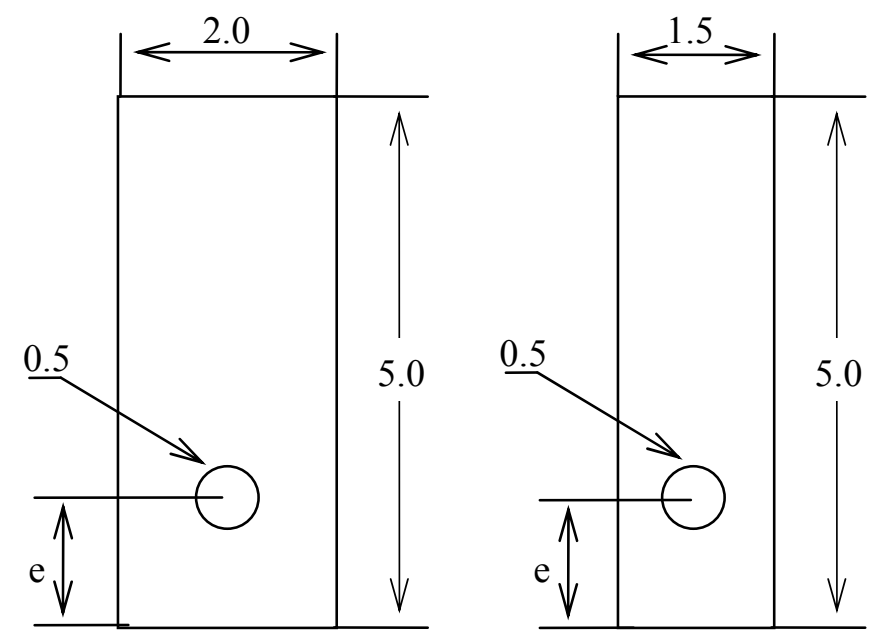

Figure 6.2 - Dimensions of specimen used in the bolted joint testing

Cutting the specimens containing reinforcing $\mathrm{SiC}$ particles using conventional carbide blades or EDM was hindered because of the abrasive properties of the $\mathrm{SiC}$. The 
$20 \% \mathrm{SiC}$ reinforced DURALCAN specimens were machined using the electrical discharge machine (EDM). The EDM was used to first cut slices from the MMC ingot and these slices were further machined into the required shape. Then, holes were drilled using cup-shaped diamond-tipped bits. Machining the MMC material, using even the EDM was difficult as the EDM requires the cut specimen to be a good conductor to make an efficient cut. The cutting speed was reduced to avoid breakage of cutting wire, which made the process very slow. To overcome this issue, polycrystalline diamond tipped tools have to be used to obtain an efficient and accurate cut with finishing operation performed on a diamond tipped grinding wheel.

Testing was conducted on double lap joint specimens loaded in the axial direction as depicted in Figure 6.3. A double-lap bolted joint testing fixture was designed as schematically represented in Figure 6-3 to facilitate the testing process. Such a fixture allows the analysis of various failures expected during tensile loading of double lap joint.

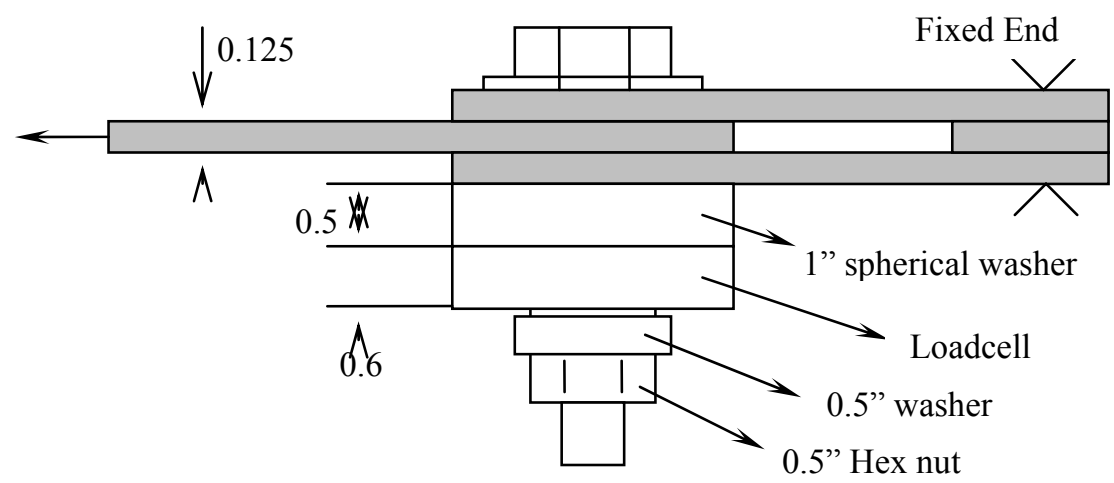

Figure 6.3 - Schematic of the test fixture used in the bolted joint testing of MMC specimen

The specimen was clamped between two steel plates as shown in Figure 6-3, and a tension force was applied gradually at the free end, until the middle plate failed. The thickness of the steel plates was chosen to be as twice as that of the MMC specimen to ensure that failure occurs in the MMC specimens. The pre-loading in a bolt has a significant effect on the strength of the bolted joint and thus is an important consideration in the design of such joints. A clamping level of $0.22 \mathrm{kN}$ (finger tight condition) was applied on the joint. 
The clamping force was measured through a miniature load cell, connected to a digital monitor, DPM3, which had been calibrated to the characteristics needs of the load cell. To ensure that the loaded cell is loaded axially, two spherical washers were used as illustrated in Figure 6-3. Additionally, the washers also prevented bending, which could occur during the bolt tightening. The main purpose of the flat washers was to decrease the stress concentration around the hole.

The two ends of the bolted joint were mounted in an Instron machine and held between its wedge grips, following which, the bolted joint was loaded. The failure mode of each kind of joint was identified.

\subsection{Finite element modeling}

Since a full analytical treatment of the bolted joints problem requires considering geometrical nonlinearities, contact stresses, and frictional effects, the finite element technique represents the most reliable tool available to carry out such an investigation. Finite element models of double-lap bolted joints were developed to simulate the tests with pin-loaded joint specimens presented earlier. A parametric mesh that allows changing the model dimensions as well as material properties was created so as to easily generate multiple models having different $\mathrm{e} / \mathrm{d}$ and $\mathrm{w} / \mathrm{d}$ ratios.

Five FE models of various e/d and w/d ratios were generated for each material type. The MMC plate was modeled using shell elements while solid brick element were used to model the bolt as shown in Figure 10. Extremely fine mesh was used in the vicinity of the hole in order to capture that stress of stress concentration that develops in this region as the joint is loaded. The selection of shell element for modeling the MMC plate allows the use of features as adaptive meshing and displaying failure modes that are available in LS-DYNA. A sliding interface is defined between the bolt and the plate that accounts for the frictional effects of the joint. Node to surface contact elements are used to define the contact surface. 


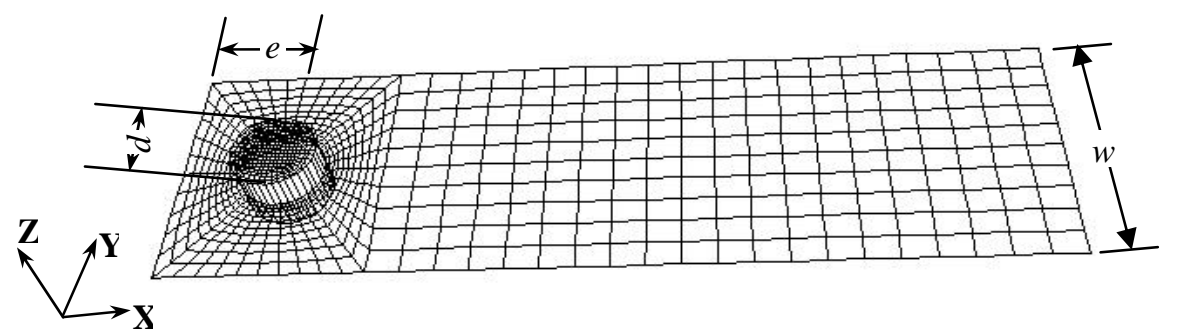

Figure 6.4 - A meshed joint with $e=0.6$ and $w=2.0$

The bolt was constrained in all its degrees of freedom and this was the only boundary condition in the model. A uniformly distrusted load was applied at the free edge of the plate to simulate the axial loading of the joint. This load magnitude is started from zero and increased gradually till the failure of the joint.

The MMC plate of the bolted joint was defined as an isotropic elastic-plastic material, while a linear-elastic material model was used for the bolt. The material parameters used in the 3DFE model for LANXIDE composites were determined by modeling the microstructure of the material as explained in the previous chapters.

\begin{tabular}{cc}
\hline Property & Value \\
\hline Density, $\mathrm{kg} / \mathrm{m}^{3}$ & 2950 \\
Young's Modulus, GPa & 98.6 \\
Yield Strength, $\mathrm{MPa}$ & 174 \\
Ultimate Strength, $\mathrm{MPa}$ & 186 \\
Failure Strain & 0.005 \\
Poisson's Ratio & 0.28 \\
\hline
\end{tabular}

Table 6.1 - Properties used in the FE model for $20 \%$ volume fraction MMC

\subsection{Results and Discussion}

A number of specimens made of $20 \% \mathrm{SiC}$ reinforcement were tested for the sake of comparison. Figure 6-5 illustrates the measured load displacement curves of the double-lap joints made of $20 \%$ Sic reinforcement MMC. These curves were generated under displacement controlled loading for a clamping force value of $50 \mathrm{~N}$ (finger tight conditions). The results show that the load rises steadily to an ultimate value, where the 
joint fails without any yielding. One may notice that for higher values of the e/d ratio, the ultimate failure load increases with the specimen still failing in the net-section mode, but without any yielding.

The load carried by a bolted joint can be divided into three stages. Initially the applied load is transferred by the frictional force until the bolts develop direct bearing contact with the plates inside the clearance of the holes .Eventually the applied tension is carried out by bearing loads plus friction along the contact surface between the jointed plates. Slipping between the bolted plates is seen in joints having high clamping force, Most of the slip occurred between 40 and 80 percent of the ultimate strength of the material. However no slip occurs when the bolted joint is tested under finger tight conditions. This is because, under finger tight conditions there is no frictional support for the bolted joint and the slip is negligible.

As a general trend, it is seen that the clamping force increases with the increase in the axial tensile load. However, the rate of the clamping force increase is higher for specimens having low e/d ratios as can be seen in Figure 6-6.

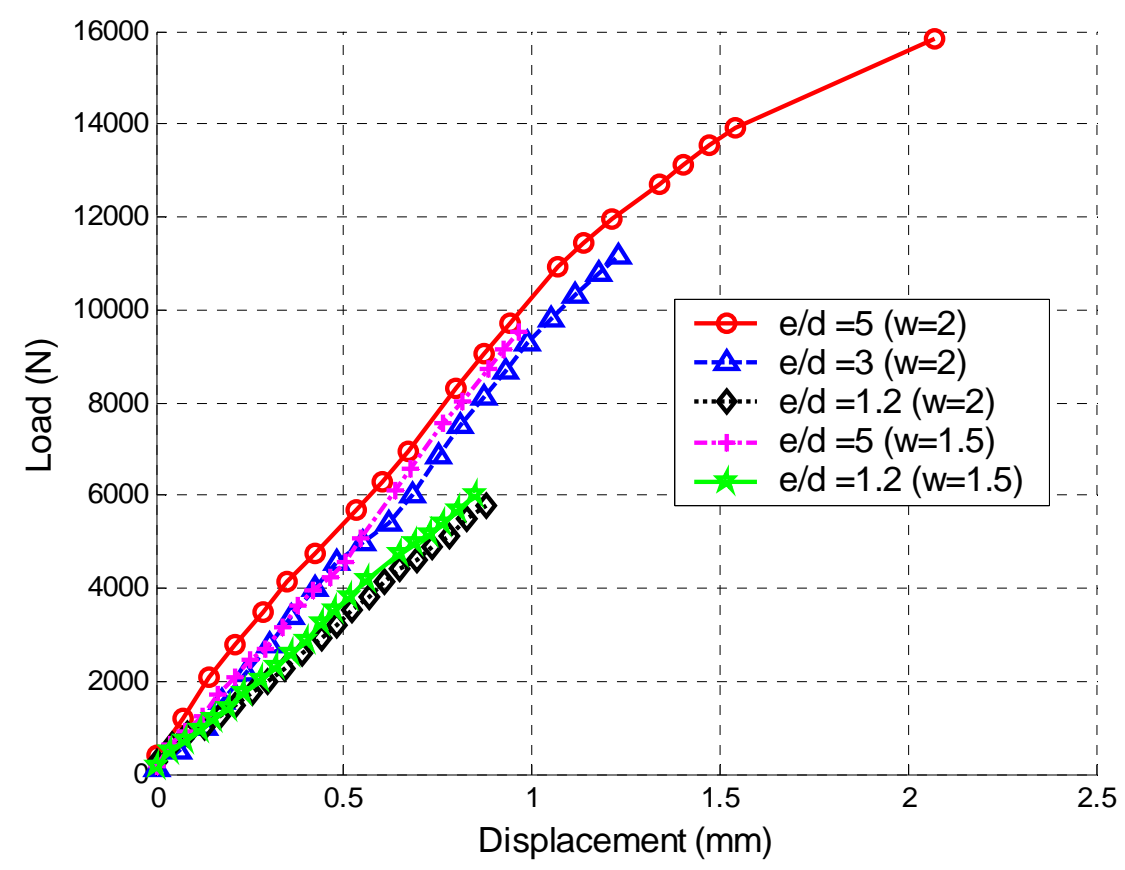

Figure 6.5 - Load -displacement curves for various joints. 


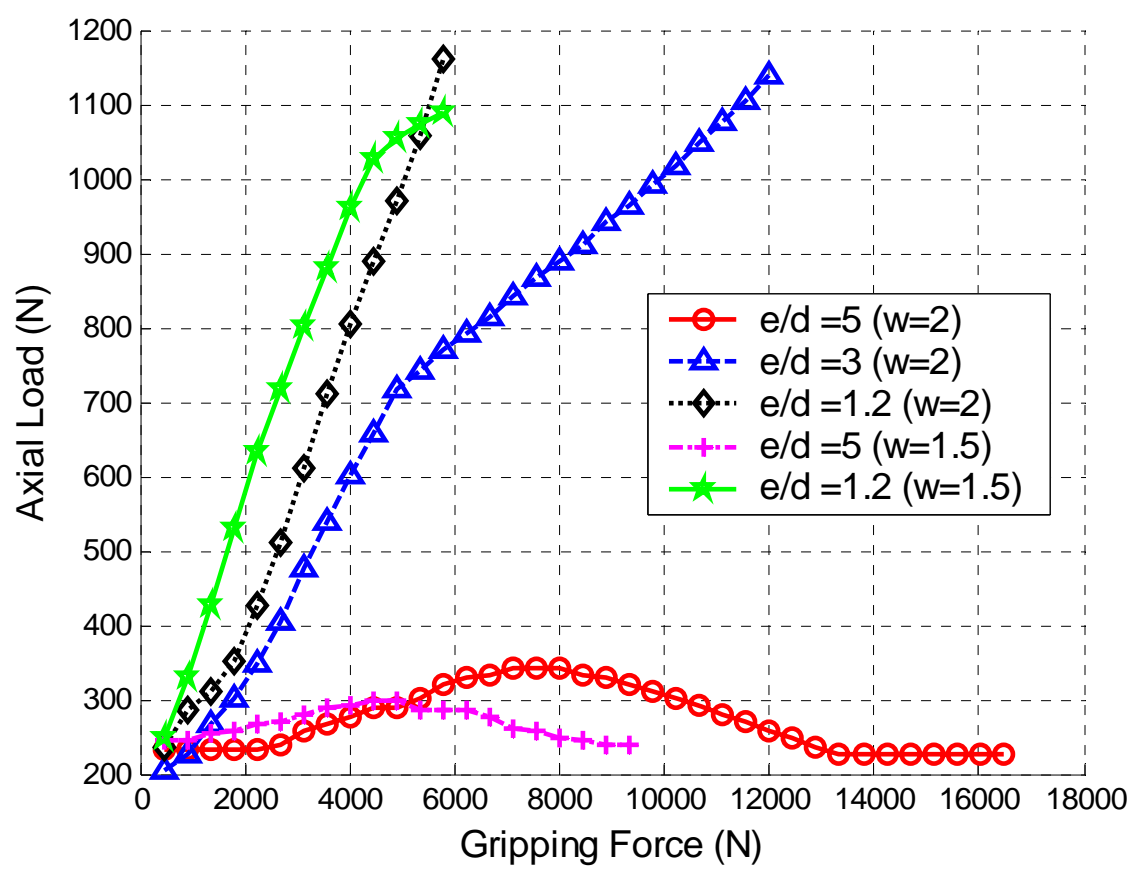

Figure 6.6 - Variation of gripping force with axial load

The two predominant failure mechanisms seen in MMC bolted joints is netsection failure and shear pull-out. The kind of failure experienced by the joint is dependent upon the $\mathrm{e}, \mathrm{d}$ and $\mathrm{w}$ values in the joint. In net-section failure, there is transverse tearing of material along the width of the joint due to either a low value of $\mathrm{w}$ or very tight bolt spacing. This type of failure is mostly seen in joints made of brittle materials. Shear pull-out occurs when the bolt tears the joint in a longitudinal direction along two parallel lines. Shear pull-out is seen in joints with low e values.

It is seen that the ultimate failure loads increase with the e/d ratios up to 5.0, and reduces with decrease in w/d ratio. This is due to the reduced ductility of the MMC material, which induces net-section failure rather than bearing failure. The failure mechanism along with failure load for each type of joint is summarized in table 6-2.

Two methods were used to predict the theoretical failure loads: 3D finite element analysis, and Euro Code standards (equations 1 and 2). Table 3 demonstrates excellent agreement between the 3DFE and experimental results for Duralcan specimens of $20 \%$ $\mathrm{SiC}$ reinforcement specimens. The difference in the 3DFE-predicted load values and the measured ones is less than $12 \%$. This is a reasonable result taking into account that the 
experimental errors in material characterization as well as the load measurement via the loading cell.

The accuracy of the 3DFE model developed for MMC bolted jointed was not only validated by the agreement between the 3DFE-calculated failure load and the experimentally measured one, but also by predicting the same mode as illustrated in Figure 6-7.

\begin{tabular}{|c|c|c|c|c|c|c|c|}
\hline \multirow{2}{*}{$\begin{array}{c}e \\
(\mathrm{~mm})\end{array}$} & \multirow{2}{*}{$\begin{array}{c}w \\
(\mathrm{~mm})\end{array}$} & \multicolumn{2}{|c|}{ Experimental } & \multicolumn{2}{|c|}{ Finite element } & \multicolumn{2}{|c|}{ Euro Standards 3} \\
\hline & & $\begin{array}{c}\text { Failure Load } \\
(\mathrm{N})\end{array}$ & Failure Mode & $\begin{array}{c}\text { Failure Load } \\
(\mathrm{N})\end{array}$ & Difference & $\begin{array}{c}\text { Failure Load } \\
(\mathrm{N})\end{array}$ & Difference \\
\hline 15 & 38 & 6,016 & Shear Pullout & 5623 & $-6.5 \%$ & 7258 & $21 \%$ \\
\hline 15 & 51 & 5,756 & Shear Pullout & 5996 & $4.2 \%$ & 7258 & $26 \%$ \\
\hline 38 & 51 & 11,192 & Net section & 12568 & $11.3 \%$ & 18114 & $62 \%$ \\
\hline 63 & 38 & 9,514 & Net section & 10498 & $10.3 \%$ & 14516 & $53 \%$ \\
\hline 63 & 51 & 15,838 & Net section & 16796 & $6.0 \%$ & 18114 & $14 \%$ \\
\hline
\end{tabular}

Table 6.2 - Failure loads for joints made of $20 \%$ SiC reinforcement

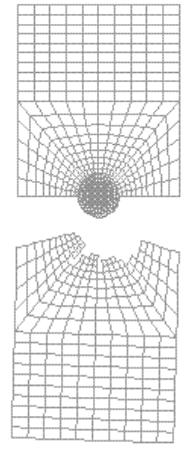

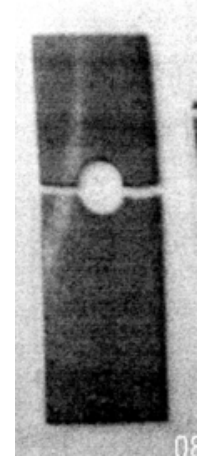

Net Section
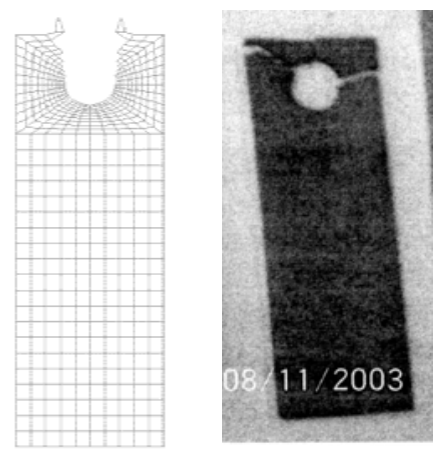

Shear Pullout

Figure 6.7 - Failure Modes of MMC Joints.

The distribution of shear stresses, $\tau \mathrm{xz}$, in a bolted joint $(\mathrm{e}=0.6, \mathrm{w}=1.5)$ made of $20 \%$ volume fraction MMC is shown in Figure 6.8(a). As the load is applied, two zones of high shear stresses form on both sides on the hole as shown. These two zones of shear stresses together with the compressive stresses at contact point with the bolt are 
responsible for the fracture of the edge of the hole. As the load is increasing applied, the failure progresses in the longitudinal direction towards the nearest joint edge as illustrated in Figure 6.8(b). The FE model is successful in predicting the same mode of failure as seen in experimental testing.

Figure 6.9(a) shows the Von-Mises effective stress distribution just as the load is applied at the plate edge for specimen with $\mathrm{e}=1.5$ and $\mathrm{w}=2.5$. Unlike the previous case, there is no stress-concentration in the area of contact of the bolt and plate. Instead, high tensile stresses are found at the top and bottom of the hole, which act as centers of failure initiation. Due to the high value of e, the failure in this case proceeds in the transverse direction, as net section failure, as shown in Figure 6.9(b). The distribution of the longitudinal stresses $\sigma_{\mathrm{x}}$ for this joint follows a similar pattern, while only their values vary.

Fringe Level, $\mathrm{MPa}$

$-10.37$

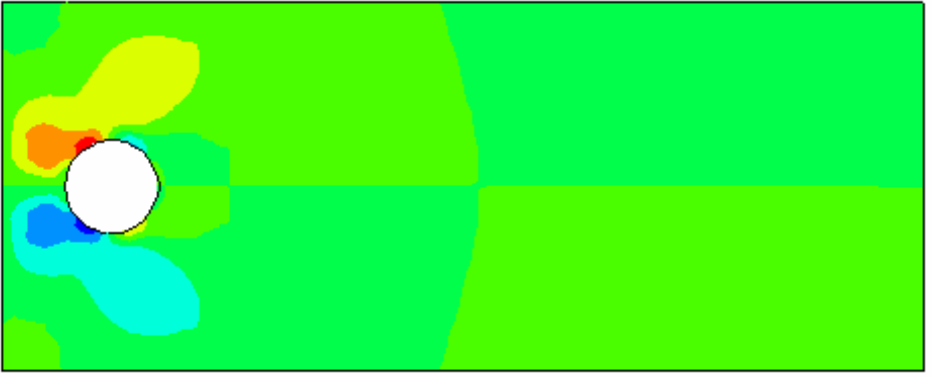

$-7.778$

$-5.185$

$-2.593$

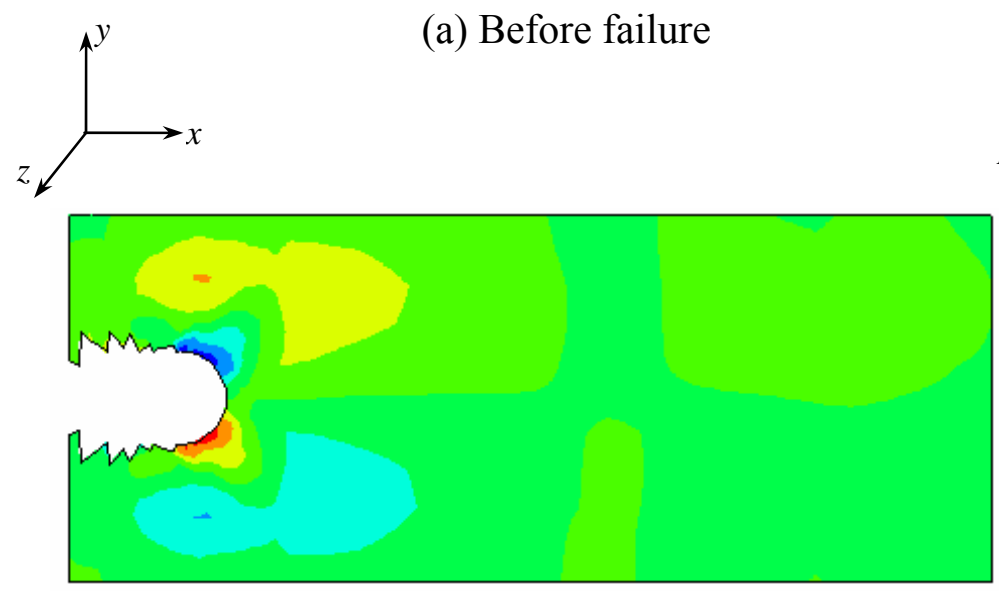

Fringe Level, $\mathrm{MPa}$

(b) At Complete

-21.12
-15.82
-10.52
-5.227
0.054
5.365
10.66
15.36
21.25

Figure 6.8 - Distribution of $\tau_{\mathrm{xy}}$ in the joint prior, at and post failure.

(e $=15 \mathrm{~mm}$ and $\mathrm{w}=\mathbf{3 8} \mathrm{mm}$ ) 
Failure Level, $\mathrm{MPa}$

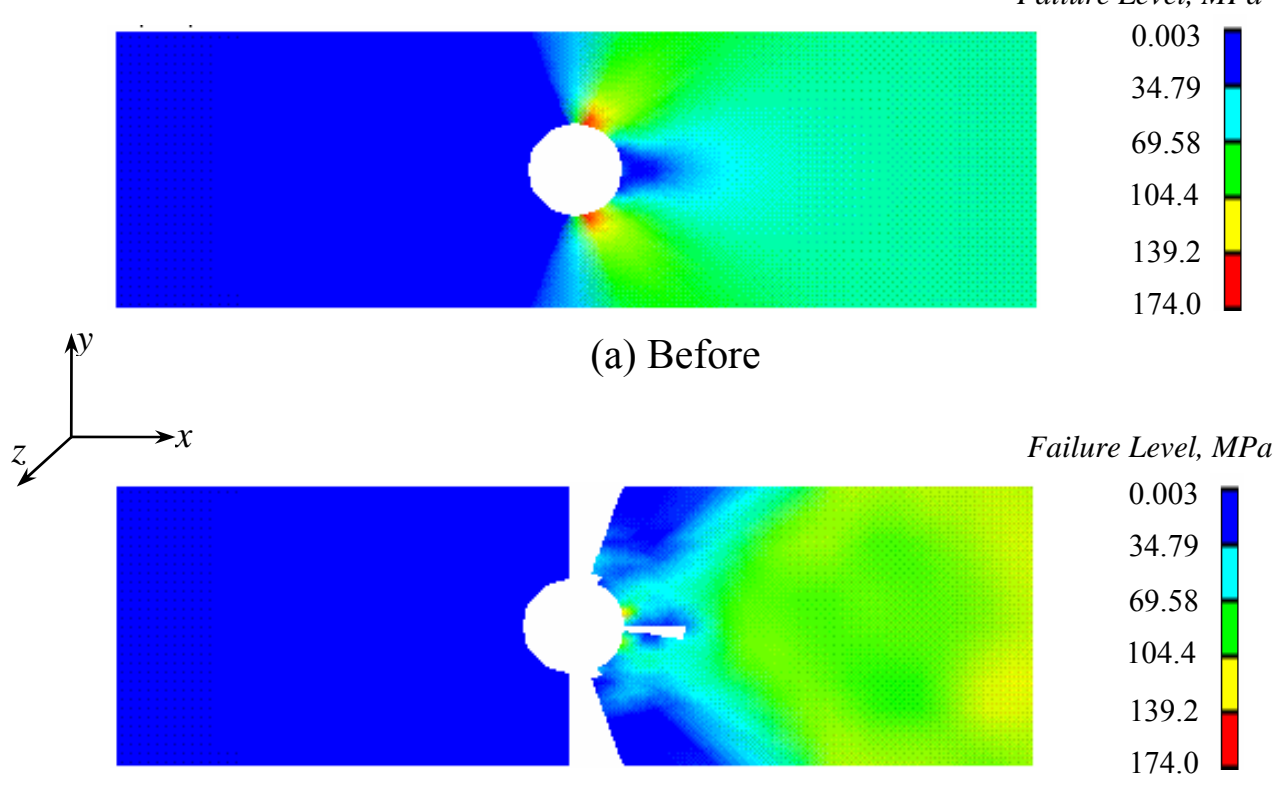

(b) Complete

Figure 6.9 - Distribution of von-Mises effective stress for a specimen having $e=2.5$ and $w$ $=1.5$ and $20 \% \mathrm{SiC}$ as reinforcement.

The finite element model uses the output of the microstructure model and is successful in predicting the mode of failure of the bolted joint along with the joint strength. 


\section{Chapter Seven}

\section{Conclusion and Recommendations}

New representative volume elements (RVE) called Random Distribution Model, incorporating spheres and ellipsoids, have been developed to investigate the properties particulate reinforced metal matrix composites. The new RVE is intended to overcome the short-comings of traditional periodic array models which idealize the microstructure of PRMMCs. The main feature of the new RVE is that it makes very few simplifying assumptions about the distribution of inclusions in the matrix. Metrics measured from micrographs of the material are used in the generation of RDMs. A mesoscale model is developed in order to account for the particle clustering effect in MMC materials.

The responses of the new models have been compared with a traditional unit cell model and values reported in the literature. It is seen that the results of the new models show better agreement with the expected value than the traditional unit cell models. these results are further applied in modeling an everyday application of MMCs, namely bolted joints. It is seen that the bolted joint FE model not only predicts the strength of the joint with reasonable accuracy, but is also successful in identifying the model of failure.

\subsection{Conclusions}

- Traditional unit cell models are not suitable for representing particulate reinforced MMCs due to the highly 3 dimensional nature of the material. The newer techniques used for exact replication of microstructure are often tedious and expensive.

- $\quad$ Computer generated microstructures offer middle-path, which is ideal for efficient generation of artificial microstructures, which can be quantified as being close to the real microstructure. 
- Simple algorithms can be used to for microstructure generation, but the problem of particle intersection or intersecting volume determination is of importance.

- Models generated in this manner on analysis reveal stress distributions which are not seen in traditional unit cell model, such as non-symmetric stress distributions.

- The RDMs predict failure to initiate just outside the matrix-reinforcement interface, while the UCMs indicate yielding in the matrix, away from the inclusion.

- Low stress bands around the particle and oriented in direction of the major axis of the ellipsoid is seen in RDMs. These low stress bands are accompanied by points of high stress concentration, which cause failure.

- Though all models, including UCMs are successful in accurately predicting the modulus of the material, the mesoscale model comes closest to the reported value of strength.

- The effect of amount of clustering on the materials properties was investigated using the mesoscale model, which shows that an increase in clustering results in an initial increase in the modulus of the material, while the yield strength remains nearly the same. An expected reduction is yield strength was not seen due to the non-inclusion of damage initiation at clustered regions of the material.

- A decrease in particle size showed an increase in the strength of the composite due to better transfer of load from the matrix to the reinforcement.

- $\quad$ The results from the microstructure model can be used as input into other model which use MMC materials, as was done in the bolted joints model to obtain a reasonably accurate estimate of joint strength. 


\subsection{Future Work}

The three models studied here can be improved in various ways to better represent the composite.

- More metrics obtained from analyzing micrographs of the material can be incorporated in the algorithm to better the model. Particle size distributions, variation in nearest neighbor distances are some of the measurements that can in included.

- A numerical technique which can measure the volume of intersection between two particles can be implemented to generated models which allow intersecting particles.

- Improved algorithms which include intersecting particles can be used to achieve higher volume fractions $(>45 \%)$

- $\quad$ Inclusion of residual stresses and imperfect particle-matrix interface in the RDMs.

The modeling technique used to represent the microstructure of PRMMCs has great potential for application in various micromechanics problems. One such application is to include equation of continuous damage mechanics with the model, in order to predict the durability of the composite. With a slight change in the algorithm, whisker reinforced MMCs can be modeled. These and other improvements are left for future researchers to investigate. 


\section{References}

[1] Bruzzi, M.S; McHugh, P.E; O’Rouke, F; Linder, T (2001) - Micromechanical modeling of the static and cyclic loading of an Al 2124-SiC MMC; International Journal of plasticity 17, p565-599

[2] Shen, H; Lissenden, C.J (2002) - 3D finite element analysis of particle reinforced aluminum; Material Science and engineering A338, p271-281

[3] Huang, H; Bush, M.B (1997) - Finite element analysis of mechanical properties of discontinuously reinforced metal matrix composites with ultra-fine microstructure; Material science and engineering A232, p63-72

[4] Williamson, G.K; Hall, W.H (1953) - Acta Metallurgica, v1, n22

[5] Leggoe, J.W; Mammoli, A.A; Bush, M.B; Hu, X.Z (1998) - Finite element modeling of deformation in particulate reinforced metal matrix composites with random local microstructure variation; Acta mater. v46 n17, p6075-6088

[6] Kenesei, P; Borbely, A; Biermann, H (2004) - Microstructure based three-dimensional finite element modeling of particulate reinforced metal-matrix composites; Material science and engineering, v387-389 n1-2, 387-389

[7] Bohm, H.J; Eckschlager, A; Han, W (2002) - Multi inclusion unit cell models for metal matrix composites with randomly oriented discontinuous reinforcements; Computational material science, v25, p42-53

[8] Baptiste, D (2003) - Nonlinear behavior micromechanical multiscale modeling of discontinuous reinforced composites; Material science forum; 426, p3939-3944

[9] Taggart, D.G; Qin, J; Adley, M.D (1992) - Evaluation of analytical and numerical models for the elastic-plastic response of particulate reinforced composites; Processing, fabrication and manufacturing of composite materials ASME; p187-200

[10] Mori, T; Tanaka, K. (1973) - Average stress in matrix and average elastic energy of materials with misfitting inclusions; Acta Metallurgica; v21 n5; p 571-574

[11] Weibull, W (1952) - A survey of statistical effects in the field of material failure; Applied Mechanical Review; v5, n11, p449-451

[12] Voyiadjis, G.Z; Dorgan, R.J; Dorrah, J.R (2001) - Bridging length scale between macroscopic response and microstructure through gradient anisotropic damage for MMC; Engineering technology conference on Energy, 2001; p643-648 
[13] Schmauder, S (2002) - Computational mechanics - Review of material science; v32, p437-465

[14] Li, M; Ghosh, S; Richmond, O; Weiland, H; Rouns, T.N (1998) - Three dimensional characterization and modeling of particle reinforced metal matrix composites: Part I Material Science and engineering. A. Structural Materials; A265, n1-2, p153-173

[15] Ghosh, S; Li, M; Moorthy, S; Lee, K - Microstructural characterization, meso-scale modeling and multiple scale analysis of discretely reinforced materials; Material science and engineering, A249 (1998), p62-70

[16] Ghosh, S; Novack, Z; Lee, K - Quantitative characterization and modeling of composite microstructures by voronoi cells; Acta mater., v45 n6, (1997) p2215-2234

[18] Li, M; Ghosh, S; Rouns, T.N; Weiland, H; Richmond, O; Hunt, W - Serial sectioning method in construction of 3D microstructures for particle reinforced MMCs; Materials characterization, v45 n2-3, (1998) p81-95

[19] Grujicic, M; Zhang, Y (1998) - Determination of effective elastic properties of functionally graded materials using Voronoi cell finite element method - Material Science and engineering. A. Structural materials; A251, n1-2, p64-76

[20] Raghavan, P; Li, S; Ghosh, S (2004) - Two scale response and damage modeling of composite materials - International journal of applied finite elements and computer aided engineering; v40 n12; p1619-1640

[21] Cottet, A (2003) - Modeling of ceramic matrix composite microstructure using a 2-D fractal spatial particle distribution - Thesis submitted to Georgia Institute of technology

[22] Xia, W; Curtin, W.A; Peters, P.W.M (2001) - Multiscale modeling of failure in metal matrix composites - Acta Materialia; v49 n2; p273-287

[23] Ramadan, H.M; Mourad, S.A; Rashed, A.A; Bode, H (1998) - Finite element modeling and experimental testing of single shear bolted joints - Advances in civil and structural engineering computing for Practice; $\mathrm{p}$ 117-24.

[24] Laviolette, D; Nica, A; Chaaban, A; Marchand, L; Shirazi-Adi, A (1996) - Mechanical behavior of pressurized bolted joints subjected to external bending loads - Proceedings of the international conference on pressure vessel technology; p117-122

[25] Lin, H; Reddy, H; McKee, R.B (1995) - Three dimensional finite element analysis of a bolted joint - The 1995 joint ASME/JSME pressure vessels and piping conference, Honolulu Hawaii; p93-98

[26] Soni, S.R; Kilic, H; Micheal, C; Derriso, M.M; Cunnigham, S (2004) - Failure analysis and behavior of titanium alloy metal matrix composites - International journal of materials and product technology; v21 n1-3; p41-58 
[27] Pratt, John D.; Pardoen (2002) - Comparative behavior of single-bolted and dualbolted lap joints - Journal of Aerospace Engineering; v 15 n2; p55-63

[28] Chung, K.F; Ip, K.H (2001) - Finite element investigation on the structural behavior of cold formed steel bolted connections - Engineering Structures, v23 n9, p1115-1125

[29] Ju, S.-H.; Fan, C.-Y.; Wu, G.H. (2004) - Three-dimensional finite elements of steel bolted connections - Engineering Structures; v26 n3; p403-413

[30] Bursi, O.S; Jaspart, J.P. (1997) - Calibration of a finite element model for isolated bolted end-plate steel connections - Journal of constructional steel research; v 44 n3, p225262

[31] Lowlor, V.P; McCarthy, M.A; Stanley, W.F (2002) - Experimental study on the effects of clearance on single bolt, single shear, composite bolted joints - Plastics, rubber and composites; $\mathrm{v} 31 \mathrm{n} 9 ; \mathrm{p} 405-411$

[32] Alblas, J.B.; Kuipers, M. - Applied Scientific Research, v 26 n 5; p 361-73.

[33] Hill, R - A self-consistent mechanics of composite materials - Journal of mechanics and physics of solids; v13; 1965, p213-222

[34] McLaughlin, R (1977) - A Study of differential scheme of composite materials International Journal of engineering science; v15; p237-244

[35] Christensen, R.M; Lo, K.H (1979) - Solutions for effective shear properties for three phase sphere and cylinder models - Journals of mechanics and physics of solids; v27; p315330

[36] Dewey, J.M (1947) - The elastic constants of materials loaded with non-rigid fillers journal of applied physics; v18; p578

[37] Mori, T; Tanaka, K (1973) - Average stress in matrix and average elastic energy of materials with misfitting inclusions - Acta metallurgica; v21; p571-574

[38] Shore, Haim (1997) - Enhancement for two commonly-used approximations for the inverse cumulative function of the normal distribution - Communications in Statistics. Part B: Simulation and Computation; v 26 n 3; p1041-1047

[39] Wang, H.Y.; Jiang, Q.C.; Wang, Y.; Ma, B.X.; Zhao, F. (2004) - Fabrication of TiB2 particulate reinforced magnesium matrix composites by powder metallurgy - Materials Letters; v 58 n 27-28; p 3509-3513

[40] Bowyer, A. (1981) - Computing Dirichlet Tessellations. - Computer Journal; v24 n 2; p162-166 
[41] Pian, T.H.H. (2000) - Some notes on the early history of hybrid stress finite element method - International Journal for Numerical Methods in Engineering; v47 n1-3; p 419-425

[42] Frame, M; Angers, M (1994) - Some nonlinear iterated function systems - Computers $\mathcal{E}$ Graphics; v 18 n1; p 119-125

[43] Upadhyaya, G.S. (1995) - Particulate metal matrix composites through powder metallurgy: some specific systems - Key Engineering Materials; v104-107 pt.1; p141-54

[44] Lee, Seong-Hee; Sakai, Tetsuo; Saito, Yoshihiro (1998) - Fabrication of Al/Al2O3 particle reinforced metal matrix composite by sheath rolling of powder mixture Materials Transactions, v39 n12; p1206-1213

[45] Hashim, J.; Looney, L.; Hashmi, (1999) - Metal matrix composites: production by the stir casting method - Journal of Materials Processing Technology; v92-93; p 1-7

[46] TrueGrid - Manual Version 2.1.0; XYZ Scientific Applications, Inc., 2001

[47] ANSYS v8.0 Structural Analysis Guide; ANSYS, Inc., 2002

[48] Liu, H.T.; Sun, L.Z.; Wu, H.C. (2005) - Monte Carlo Simulation of particle-cracking damage evolution in metal matrix composites - Transactions of the ASME. Journal of Engineering Materials and Technology, v127 n3; p318-24

[49] Bochenek, B; Pyrz, R (2004) - Reconstruction of random microstructures A stochastic optimization problem - Computational Materials Science, v31 n1-2; p93-112

[50] Bohm, H.J; Eckschlager, A.; Han, W (2002) - Multi-inclusion unit cell models for metal matrix composites with randomly oriented discontinuous reinforcements Computational Materials Science; v25 n1-2; p42-53

[51] Using Matlab 6.5; The Mathworks Inc., 2004

[52] StrainSmart® Software Specifications/Features - Version 3.1; Vishay Micromeasurements

[53] Particulate reinforced metal matrix composites, A review (1991) - Ibrahim, I.A; Journal of material science; v26 n5, p1137

[54] Longsdon, W.A (1986); Engineering fracture mechanics; v24 n5; 737-751

[55] Garneau, J.-F.; Angers, R.; Krishnadev, M.R.; Collins, L. (1993) - Fabrication and characterization of $\mathrm{SiC} / 6061$ composites - Developments and applications of ceramics and new metal alloys : proceedings of the International Symposium on Developments and Applications of Ceramics and New Metal Alloys; Quebec City, Quebec, August 29September 2; p26-33 
[56] Doel, T.J.A. - Tensile properties of particulate-reinforced metal matrix composites Composites. Part A, Applied science and manufacturing; v27 n8; p655-665

[57] Srivatsan, T.S (1996) - Microstructure, tensile properties and fracture behavior of $\mathrm{Al}_{2} \mathrm{O}_{3}$ particulate-reinforced aluminum alloy metal matrix composites - Journal of material science; v31 n5-6; 1375 


\section{Appendix}

\subsection{Matlab routine to generate microstructures with sphere-shaped inclusions}

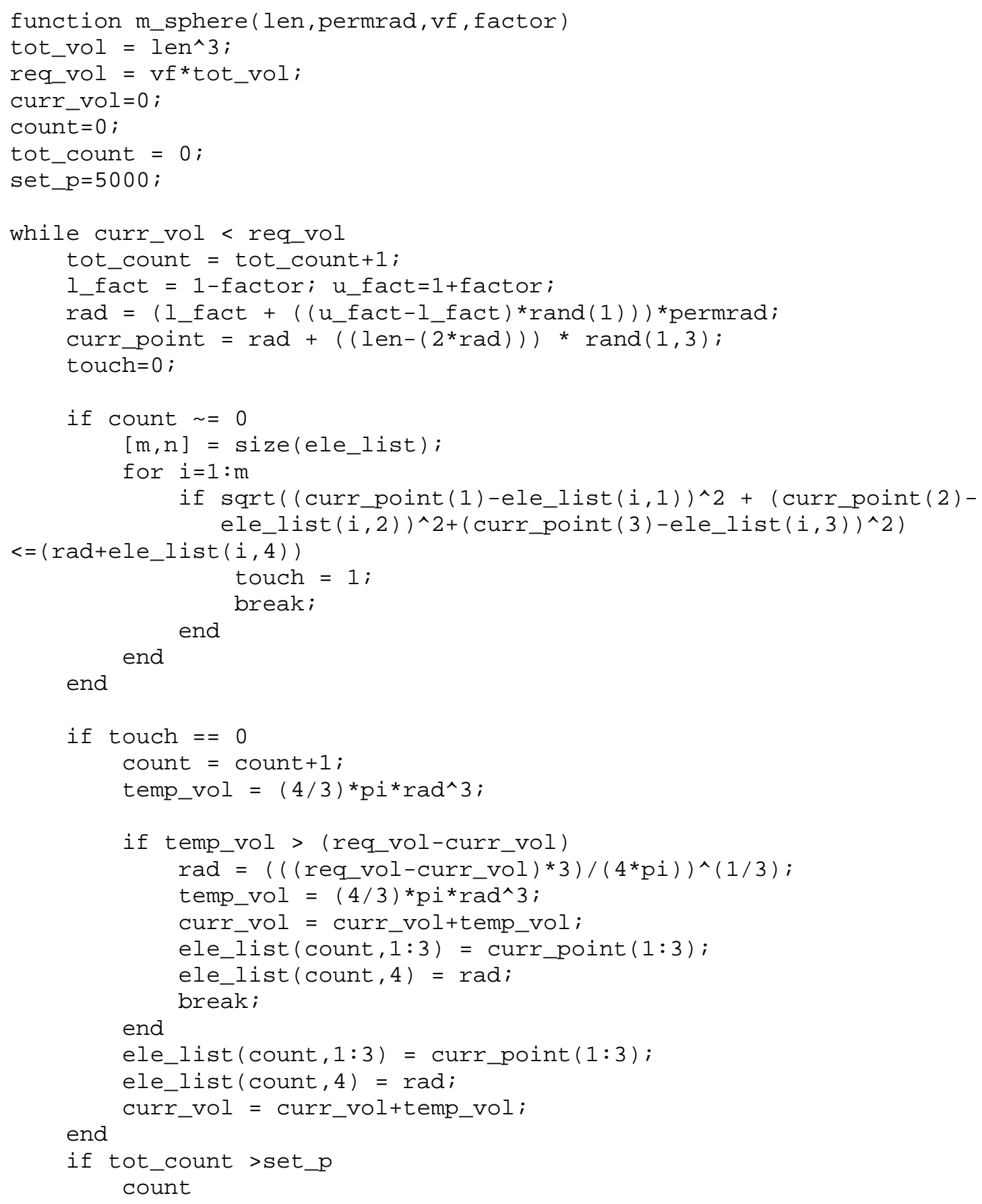




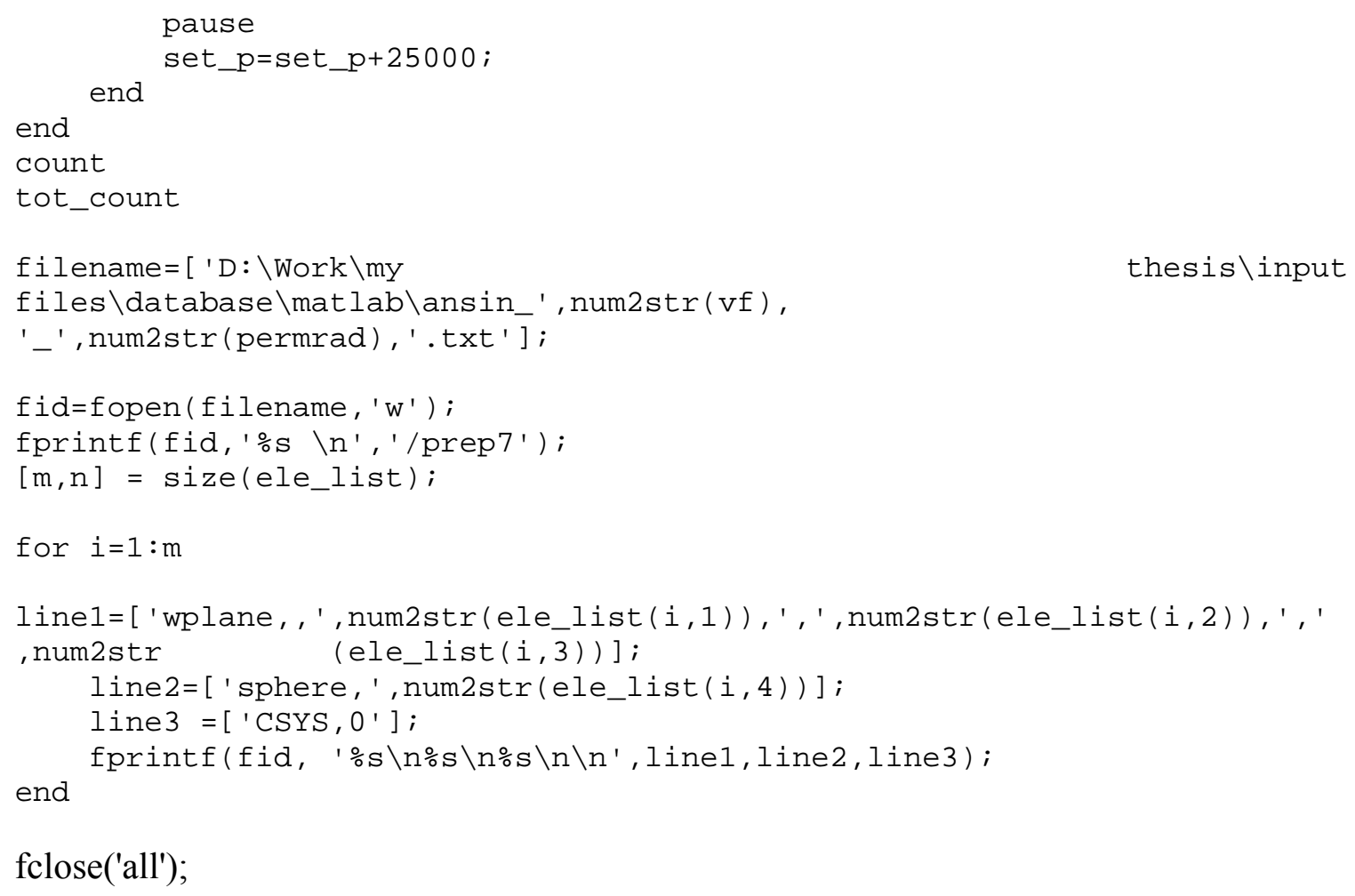

fclose('all');

\subsection{Matlab routine to generate microstructures with ellipsoid- shaped inclusions}

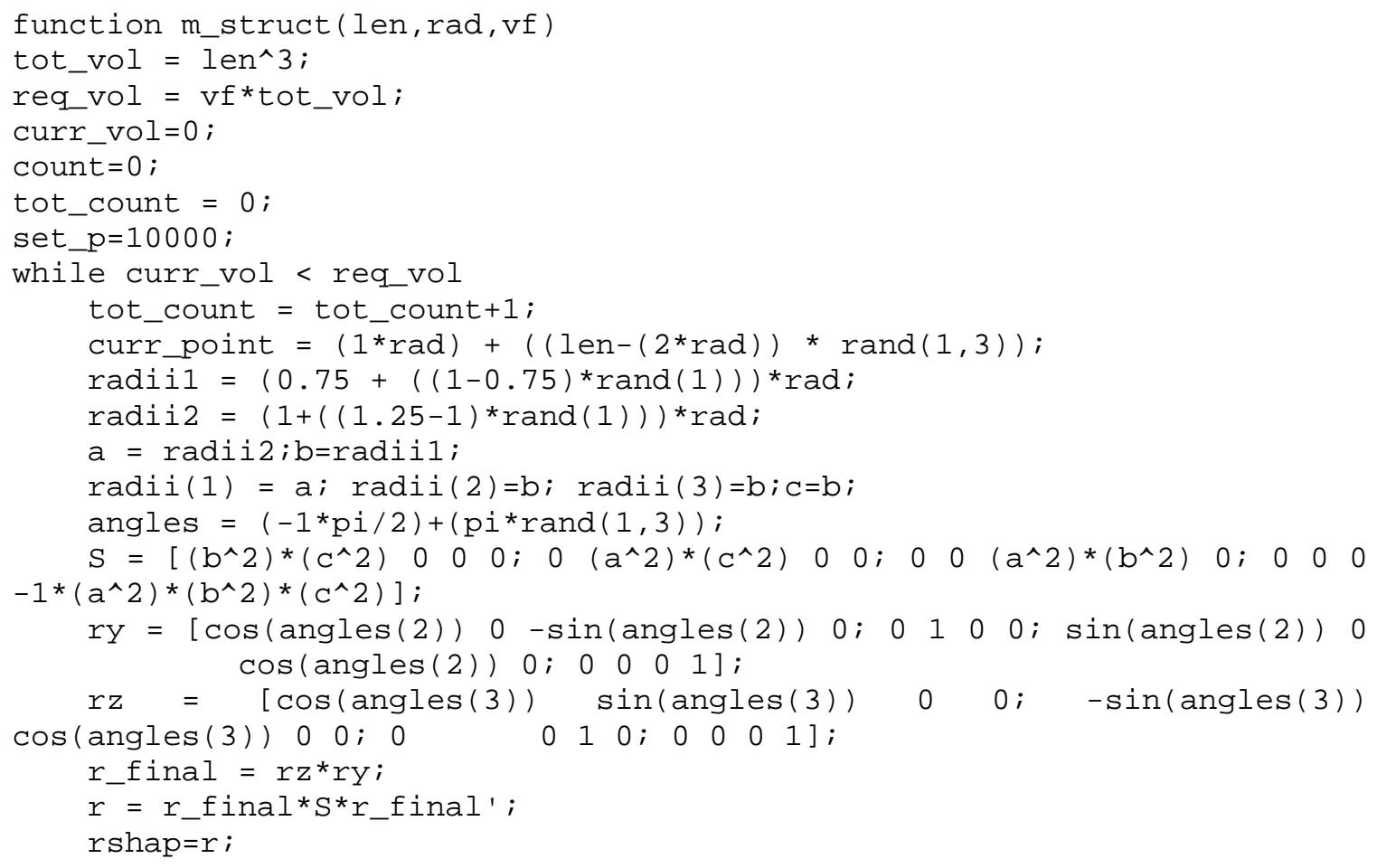




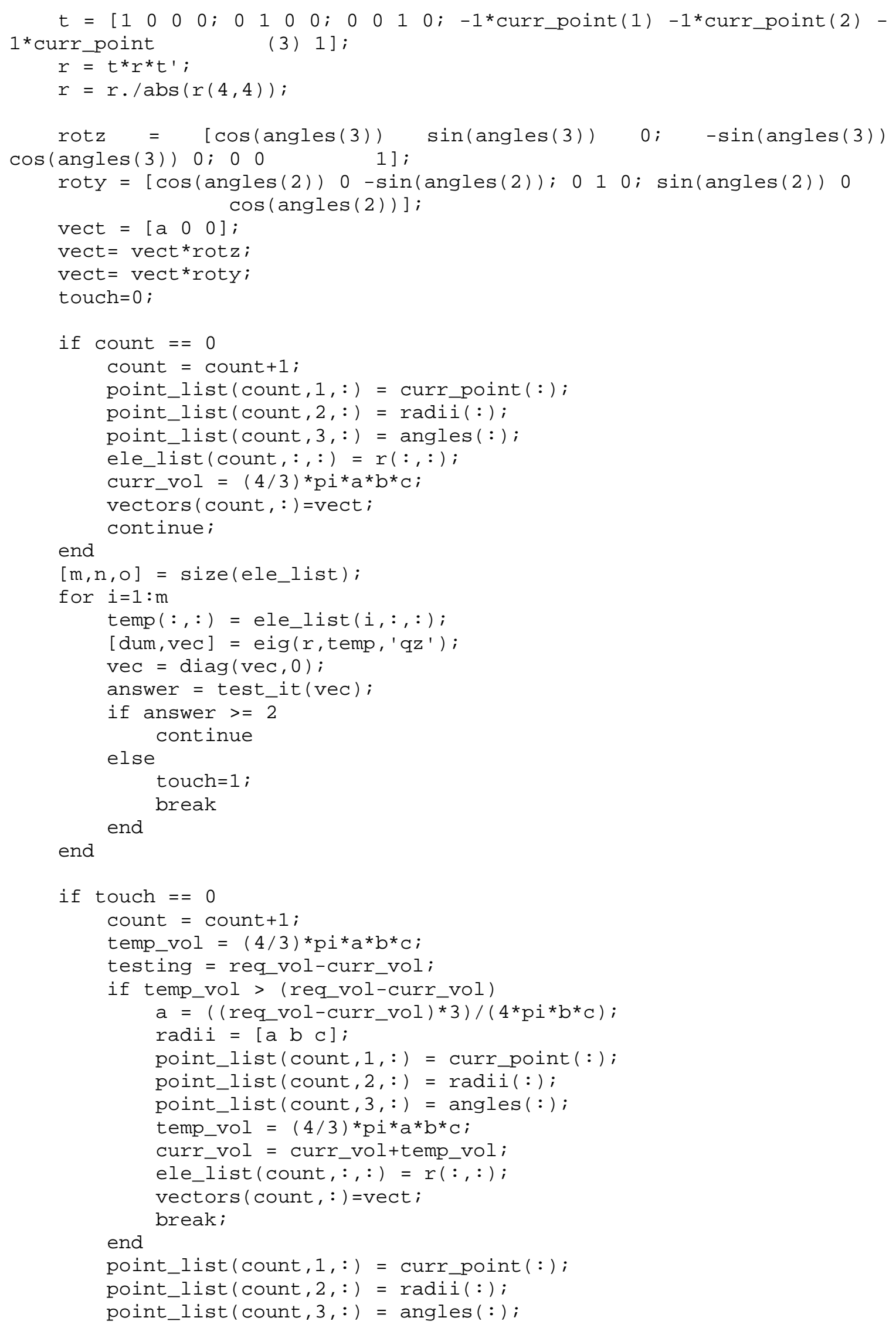




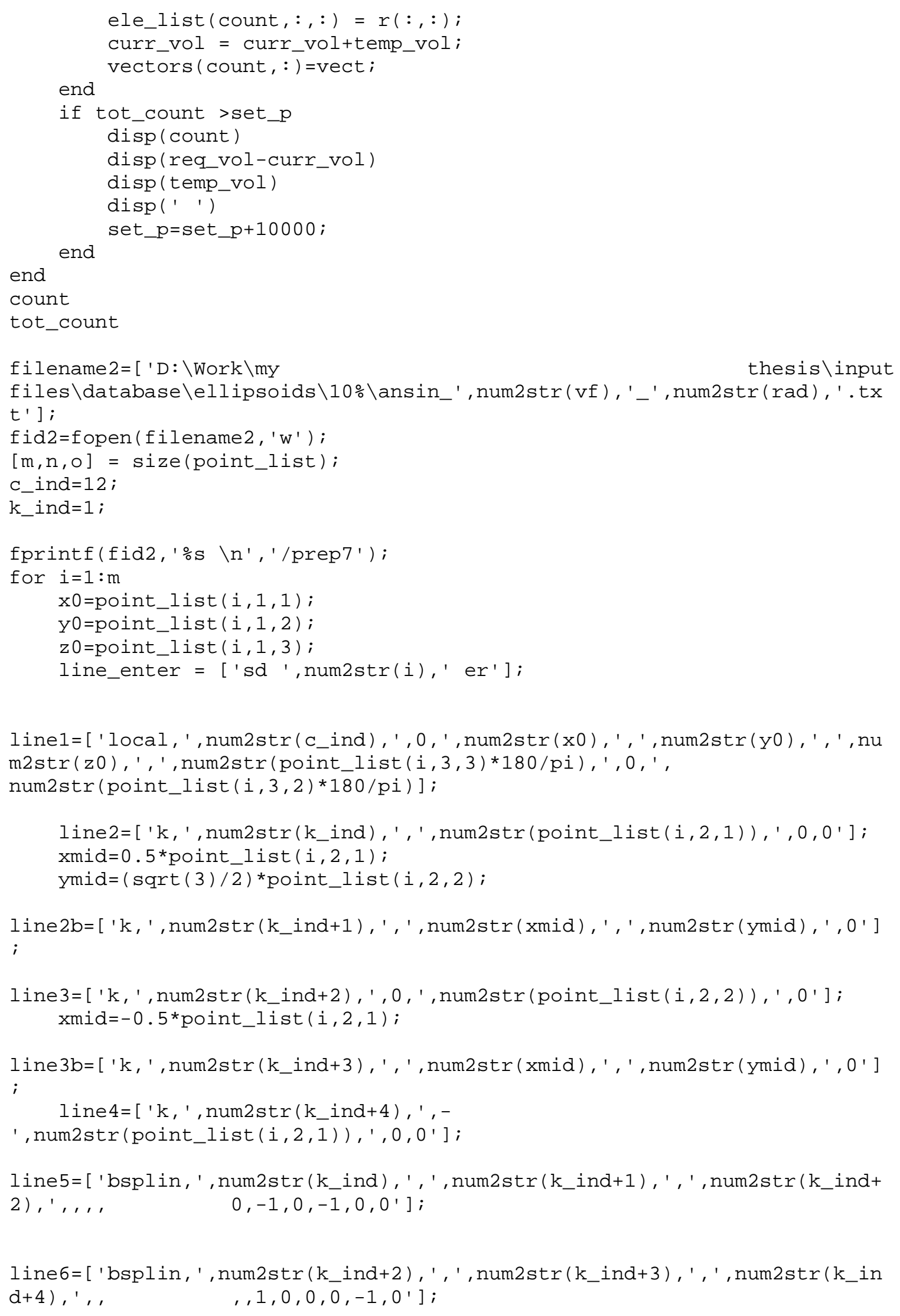


line $7=\left[{ }^{\prime} 1\right.$, ', num2str $\left(k \_i n d+4\right), '$, ', num2str(k_ind $\left.)\right]$;

$\mathrm{k} \_$ind $=\mathrm{k} \_$ind +5 ;

fprintf(fid2, '\%s \n \%s \n \%s \n \%s \n \%s \n \%s \n \%s \n \%s \n

\%s $\backslash n$ ', line1, line2, line2b, line3, line3b, line4, line5, line6, line7)；

end

k_ind $=1$;

1_ind $=1$;

fprintf(fid2, '\n');

for $i=1: m$

line8 $=\left[\right.$ 'al, ' , num2str $\left(1 \_\right.$ind $),{ }^{\prime}$, ' , num2str $\left(1 \_\right.$ind +1$)$, ' , ' , num2str $\left(1 \_\right.$ind +2$\left.)\right]$; fprintf(fid2, '\%s\n ', line8);

end $1 \_$ind $=1 \_$ind +3 ;

k_ind $=1$;

a_ind $=1$;

fprintf(fid2, '\n');

for $i=1: m$

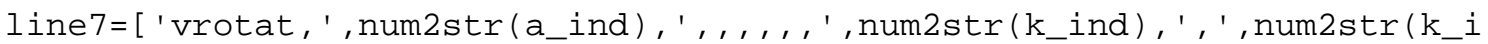
$\mathrm{nd}+4)]$;

fprintf(fid2, '\%s \n ', line7);

a_ind $=$ a_ind +1 ;

end

$\mathrm{k} \_$ind $=\mathrm{k} \_$ind +5 ;

fclose('all');

\subsection{Example ANSYS macro file used to specify material properties, loading, boundary conditions and post processing}

/prep7

len $=70$

$\mathrm{mp}, \mathrm{ex}, 1,70 \mathrm{e}-3$

$\mathrm{mp}$, nuxy, $1,0.33$

TB, MISO, 1, 1, 13

tbpt, DEFI, 3.619e-3,250e-6

tbpt, DEFI, 3.965e-3, 264.13e-6

tbpt, DEFI, 4.582e-3,272.75e-6

tbpt, DEFI, 5.333e-3, 277.30e-6

tbpt, DEFI, 6.176e-3, 279.65e-6

tbpt, DEFI, 7.244e-3, 281.92e-6

tbpt, DEFI, 8.132e-3, 283. 02e-6

tbpt, DEFI, 9.094e-3, 284.2e-6

$m p, e x, 2,410 e-3$ 
mp, nuxy, 2, 0.14

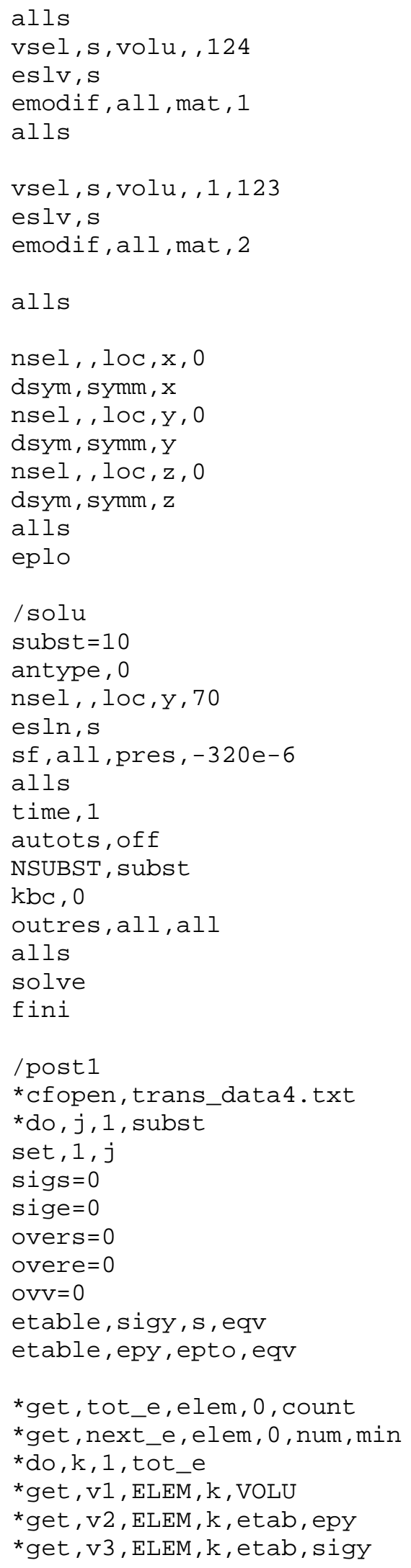


sigs $=\operatorname{sigs}+\left(v 1^{*} v 3\right)$

sige $=\operatorname{sige}+(\mathrm{V} 1 * \mathrm{~V} 2)$

$o v v=o v v+v 1$

*enddo

overs=sigs/ovv

overe=sige/ovv

*vwrite, overs, overe, ovv

(E10.3, 2x, E10.3, 2x, E10.3)

*enddo

${ }^{*}$ cfclos 